University of Tennessee Health Science Center

UTHSC Digital Commons

\title{
A Dynamic Ankle Orthosis for Alleviating Mechanical Foot and Ankle Pain, Preserving Joint Motion, and Restoring Patient Function
}

Chloe Ly Chung

University of Tennessee Health Science Center

Follow this and additional works at: https://dc.uthsc.edu/dissertations

Part of the Analytical, Diagnostic and Therapeutic Techniques and Equipment Commons

\section{Recommended Citation}

Chung, Chloe Ly (http://orcid.org/0000-0002-0185-349X), "A Dynamic Ankle Orthosis for Alleviating Mechanical Foot and Ankle Pain, Preserving Joint Motion, and Restoring Patient Function" (2020). Theses and Dissertations (ETD). Paper 509. http://dx.doi.org/10.21007/etd.cghs.2020.0494. 


\title{
A Dynamic Ankle Orthosis for Alleviating Mechanical Foot and Ankle Pain, Preserving Joint Motion, and Restoring Patient Function
}

\begin{abstract}
Over two million Americans visit the doctor each year for foot and ankle pain stemming from a degenerative condition or injury. Standard of care ankle-foot orthoses have been shown to effectively manage pain symptoms of the lower limb, but they can also significantly hinder joint motion and limit function. The Dynamic Ankle Orthosis (DAO) was created with the intent to alleviate pain using an offloading mechanism without interfering with natural joint motion. This dissertation presents the development, benchtop validation, functional assessment, and clinical evaluation of this novel treatment option for patients suffering from acute and chronic foot and ankle pain.A benchtop validation study was performed to determine the offloading capacity of the DAO during static double stance and to quantify the brace's effect on passive ankle motion. Static offloading of the DAO was measured using load cells, and an isokinetic dynamometer measured resistance to passive, non-weight-bearing ankle motion with and without the brace. The DAO offloaded between $11 \%-30 \%$ BW with $77 \%-95 \%$ brace force transference. The DAO permitted a functional range of ankle motion ( $10^{\circ}$ dorsiflexion to $20^{\circ}$ plantarflexion) and did not increase resistance to ankle motion. In a gait laboratory setting, a functional assessment was performed with ten healthy subjects to compare ankle joint kinematics and plantar pressures during treadmill walking among the DAO, a standard double upright ankle-foot orthosis (DUAFO), and an unbraced control (CON) condition. Ankle kinematics were assessed using a 3D motion capture system and in-shoe plantar pressures were measured for seven areas of the foot. DAO reduced hallux peak plantar pressures compared to CON and DUAFO. Peak plantar pressures under toes 2-5 were smaller in DAO than DUAFO. Eversion ROM was much smaller in DUAFO compared to CON and DAO. The impact of the DAO on pain and function was evaluated in a clinical setting with twenty-five patients symptomatic with foot and/or ankle pain. Eight functional activities were completed with CON and DAO. Force insoles were used to capture in-shoe vertical forces and a visual analog scale was used to assess pain levels during each activity. A post-test questionnaire was used to measure the self-perceived impact of the DAO on the patient's symptoms and function. Peak in-shoe forces were reduced during level and stair walking $(p<0.05)$. Average pain was 1.2 to 1.6 points lower with DAO than CON for the active tasks (clinically meaningful). Most participants reported that the DAO either improved $(n=19)$ or did not affect their symptoms $(n=5)$. Function scores were improved on average (+3.7). DAO improved symptoms of osteoarthritis, posterior tibialis tendon dysfunction, postoperative pain, ankle sprains, and nerve disorders. This body of work demonstrates the capacity of the DAO to provide significant offloading during ambulation without greatly affecting kinematic parameters including sagittal and frontal plane ankle motion compared to an unbraced control. Additionally, this work provides novel evidence that the DAO can improve symptoms and the ability of impaired individuals to complete functional activities such as level and stair walking.
\end{abstract}

\section{Document Type}

Dissertation

\section{Degree Name}

Doctor of Philosophy (PhD)

\section{Program}

Biomedical Engineering

Research Advisor

Denis J. DiAngelo 


\section{Keywords}

Foot and Ankle, Functional, Gait, Mechanical Pain, Orthosis

\section{Subject Categories}

Analytical, Diagnostic and Therapeutic Techniques and Equipment | Medicine and Health Sciences 


\section{A Dynamic Ankle Orthosis for Alleviating Mechanical Foot and Ankle Pain, Preserving Joint Motion, and Restoring Patient Function}

Author:

Chloe Ly Chung
Advisor:

Denis J. DiAngelo, PhD

A Dissertation Presented for The Graduate Studies Council of

The University of Tennessee Health Science Center

in Partial Fulfillment of the Requirements for the

Doctor of Philosophy degree

In the Joint Graduate Program in Biomedical Engineering

From The University of Tennessee

and The University of Memphis

Biomedical Engineering: Biomechanics

College of Graduate Health Sciences

May 2020 
Chapter 4 (C) 2020 by The American Society of Mechanical Engineers. All other material (C) 2020 by Chloe Ly Chung.

All rights reserved. 


\section{DEDICATION}

For my parents, Nicole M. Arsenault and Hoang M. Chung, my little brother, Brandon M. Chung, and my best friend and companion, Tayler M. Daniels, who remind me to fearlessly challenge the status quo, to always finish what I start, and to take life one day at a time. 


\section{ACKNOWLEDGEMENTS}

I'd like to thank my advisor, Dr. Denis J. DiAngelo, who saw potential in me, made this research opportunity available, and has pushed me out of my comfort zone time and time again. I'd like to thank my committee members, Dr. Richard Kasser, Dr. Eugene Eckstein, Dr. William Mihalko, Dr. Maxime Paquette, and Dr. Douglas Powell for their guidance and support during my time at the University of Tennessee Health Science Center. I would like to broadly thank each and every person who served as a guinea pig during the development and testing of the Dynamic Ankle Orthosis (DAO): I sincerely appreciate you volunteering your time and feedback, and I am sorry about the duct tape. I would also like to acknowledge my fellow students in the UTHSC Orthopedic Biorobotics and Rehabilitation Laboratory, Michael Parker, Daniel Hoyer, and Lavanya Bharani, for their never-ending support during research and life challenges alike, and for their valuable contributions during different phases of this research. Special thanks to the students of the University of Memphis Musculoskeletal Analysis Lab, especially Ramzi Majaj, for their assistance during the functional assessment of the DAO.

Special thanks to the orthotists, Jack Steele, Cathie Baker, Terry Tate, and Jim Huddleston, at the Center for Orthotics and Prosthetics, Inc. for their guidance during the design phase of this work and for their help in planning the clinical study. I am especially grateful to Fred Williams, Justin Thomas, and Deion Jones at the Center for Orthotics and Prosthetics, Inc. for their help with the fabrication of the DAO, DUAFO, and other orthoses used in this work as well as all of the laughs along the way. Special thanks to foot and ankle surgeons Dr. Andrew Murphy, Dr. David Richardson, Dr. Clayton Bettin, and Dr. Benjamin Grear and their incredible staff at Campbell Clinic Orthopaedics, for providing specialized input and access to patients during the clinical evaluation of the DAO. Finally, I'd like to thank my family for providing me with constant support and encouragement throughout my life, and without whom I would not have the courage to continue pursuing my dreams.

This work was supported by the University of Tennessee Research Foundation Maturation Grant, "Dynamic Ankle Orthosis for Alleviating Pain and Preserving Motion," 2016-2017 and the University of Memphis Research Investment Fund, "Functional Evaluation of a New Dynamic Ankle Orthosis (DAO)," 2017-2018. 


\begin{abstract}
Over two million Americans visit the doctor each year for foot and ankle pain stemming from a degenerative condition or injury. Standard of care ankle-foot orthoses have been shown to effectively manage pain symptoms of the lower limb, but they can also significantly hinder joint motion and limit function. The Dynamic Ankle Orthosis (DAO) was created with the intent to alleviate pain using an offloading mechanism without interfering with natural joint motion. This dissertation presents the development, benchtop validation, functional assessment, and clinical evaluation of this novel treatment option for patients suffering from acute and chronic foot and ankle pain.
\end{abstract}

A benchtop validation study was performed to determine the offloading capacity of the DAO during static double stance and to quantify the brace's effect on passive ankle motion. Static offloading of the DAO was measured using load cells, and an isokinetic dynamometer measured resistance to passive, non-weight-bearing ankle motion with and without the brace. The DAO offloaded between 11\%-30\% BW with 77\%-95\% brace force transference. The DAO permitted a functional range of ankle motion $\left(10^{\circ}\right.$ dorsiflexion to $20^{\circ}$ plantarflexion) and did not increase resistance to ankle motion.

In a gait laboratory setting, a functional assessment was performed with ten healthy subjects to compare ankle joint kinematics and plantar pressures during treadmill walking among the DAO, a standard double upright ankle-foot orthosis (DUAFO), and an unbraced control (CON) condition. Ankle kinematics were assessed using a 3D motion capture system and in-shoe plantar pressures were measured for seven areas of the foot. DAO reduced hallux peak plantar pressures compared to CON and DUAFO. Peak plantar pressures under toes 2-5 were smaller in DAO than DUAFO. Eversion ROM was much smaller in DUAFO compared to CON and DAO.

The impact of the DAO on pain and function was evaluated in a clinical setting with twenty-five patients symptomatic with foot and/or ankle pain. Eight functional activities were completed with CON and DAO. Force insoles were used to capture inshoe vertical forces and a visual analog scale was used to assess pain levels during each activity. A post-test questionnaire was used to measure the self-perceived impact of the $\mathrm{DAO}$ on the patient's symptoms and function. Peak in-shoe forces were reduced during level and stair walking $(\mathrm{p}<0.05)$. Average pain was 1.2 to 1.6 points lower with DAO than $\mathrm{CON}$ for the active tasks (clinically meaningful). Most participants reported that the DAO either improved $(n=19)$ or did not affect their symptoms $(n=5)$. Function scores were improved on average (+3.7). DAO improved symptoms of osteoarthritis, posterior tibialis tendon dysfunction, postoperative pain, ankle sprains, and nerve disorders.

This body of work demonstrates the capacity of the DAO to provide significant offloading during ambulation without greatly affecting kinematic parameters including sagittal and frontal plane ankle motion compared to an unbraced control. Additionally, this work provides novel evidence that the DAO can improve symptoms and the ability of impaired individuals to complete functional activities such as level and stair walking. 


\section{TABLE OF CONTENTS}

CHAPTER 1. INTRODUCTION .......................................................................................

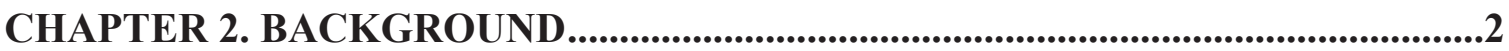

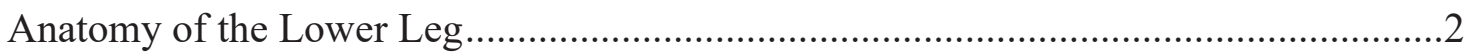

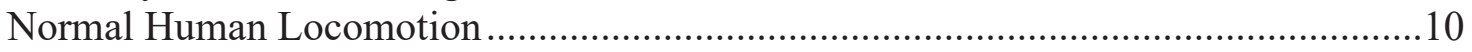

Conditions of the Lower Leg that Present Pain and Impact Function ...........................12

Acute Injuries ..............................................................................................12

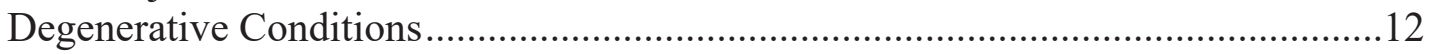

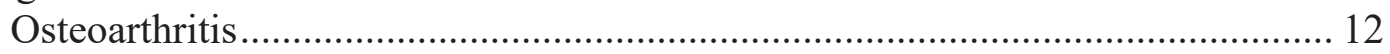

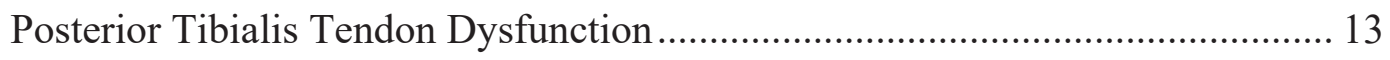

Chronic Postoperative Pain ............................................................................. 13

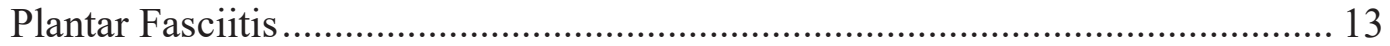

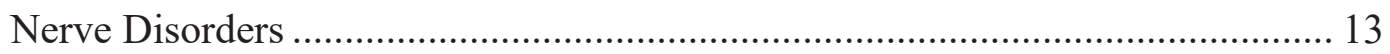

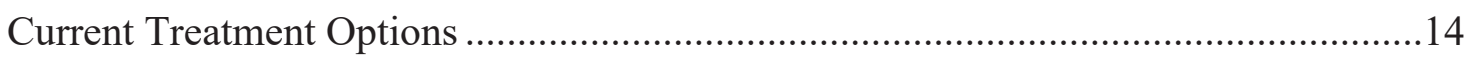

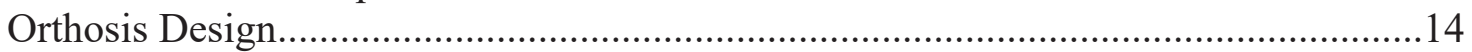

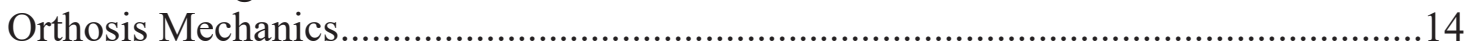

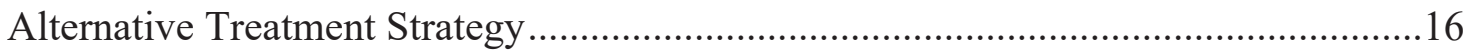

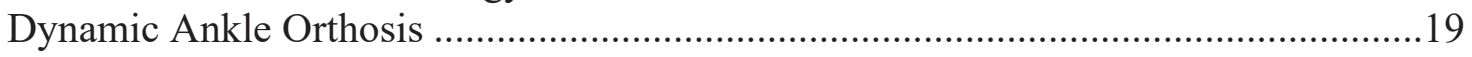

\section{CHAPTER 3. DESIGN AND VALIDATION TESTING OF A DYNAMIC}

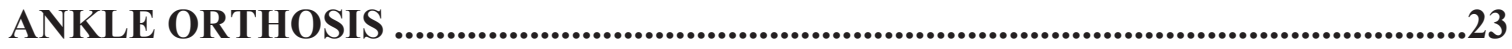

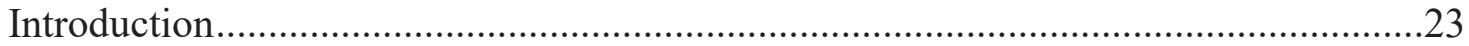

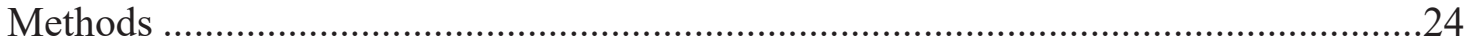

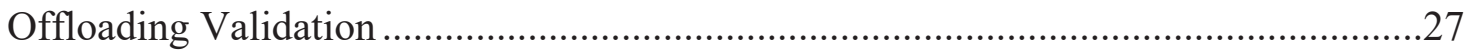

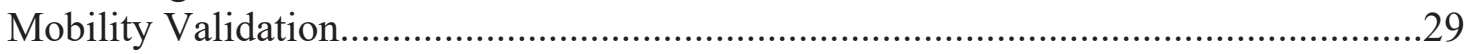

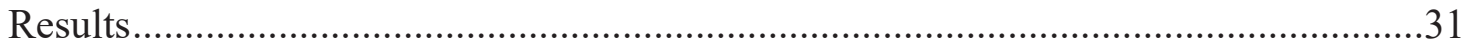

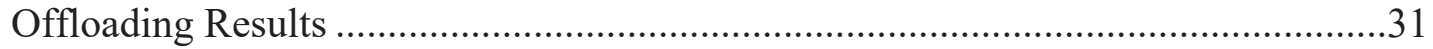

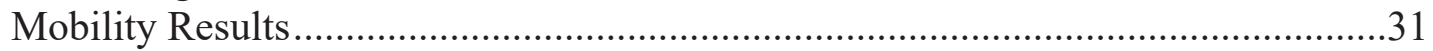

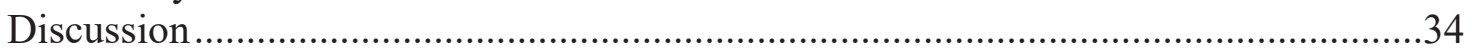

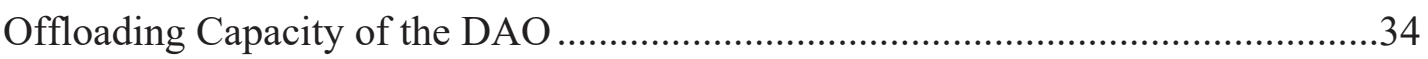

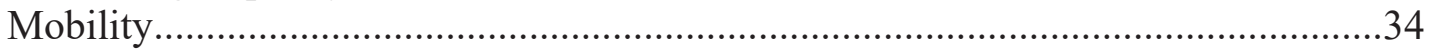

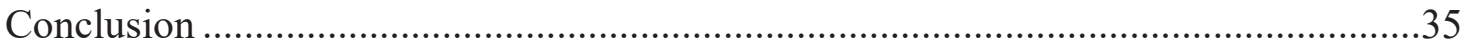

CHAPTER 4. BIOMECHANICAL COMPARISON OF A NEW DYNAMIC ANKLE ORTHOSIS TO A STANDARD ANKLE-FOOT ORTHOSIS DURING

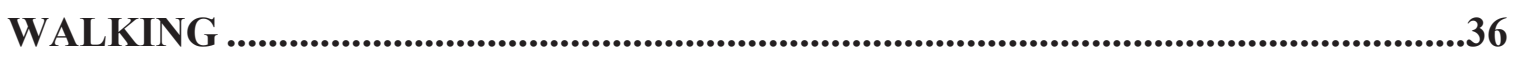

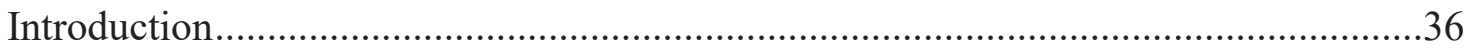

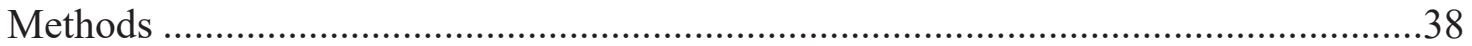

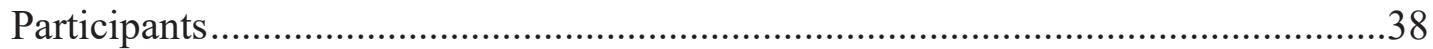

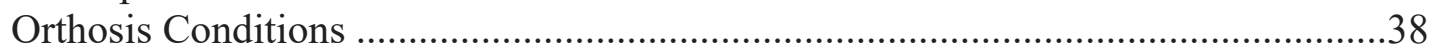

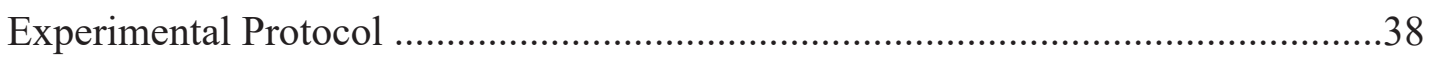

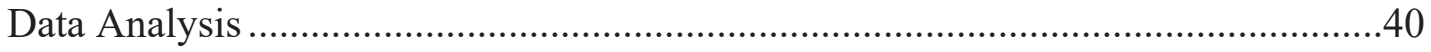

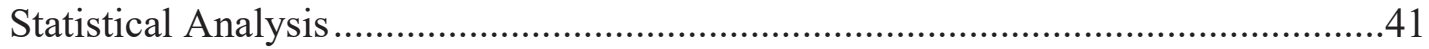


Results

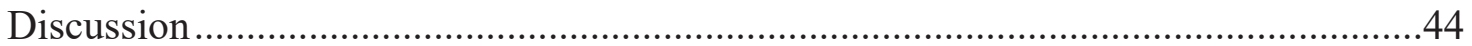

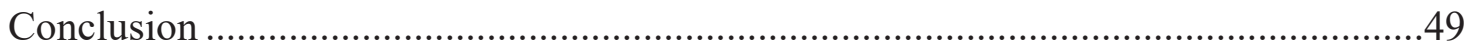

\section{CHAPTER 5. IMPACT OF THE DYNAMIC ANKLE ORTHOSIS ON} MECHANICAL PAIN AND FUNCTION IN PATIENTS WITH FOOT AND

ANKLE DISEASES .................................................................................................................50

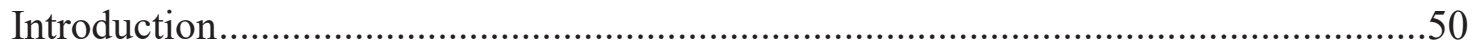

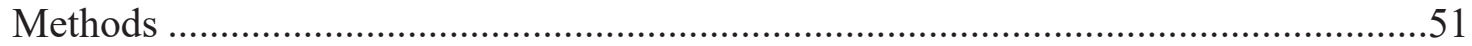

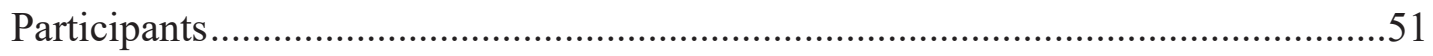

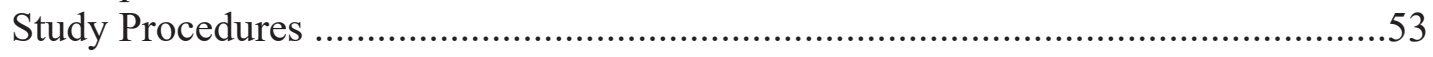

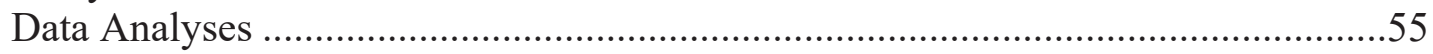

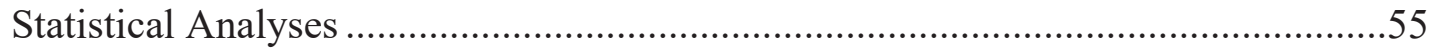

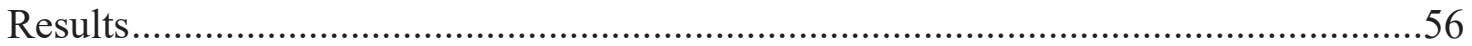

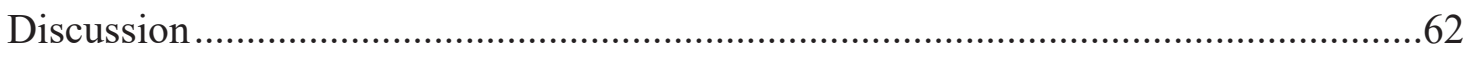

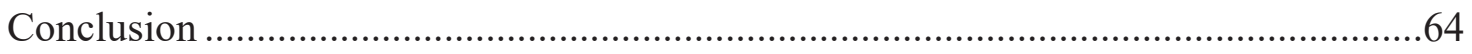

CHAPTER 6. FUTURE DIRECTIONS ...............................................................65

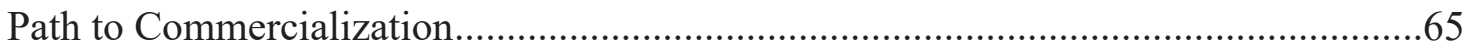

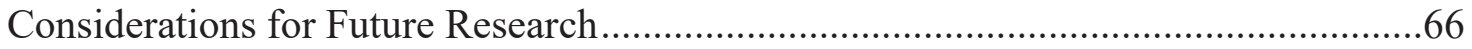

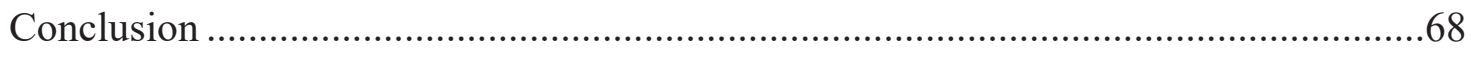

LIST OF REFERENCES .......................................................................................69

APPENDIX A. SPECIFICATIONS ..........................................................................8

APPENDIX B. PILOT STUDY COMPARING THE DAO WITH A PTB

ORTHOSIS IN OVER-GROUND WALKING ...........................................................83

APPENDIX C. EXTENDED METHODOLOGY OF THE FUNCTIONAL

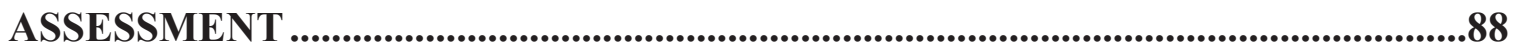

APPENDIX D. EXTENDED RESULTS OF THE FUNCTIONAL

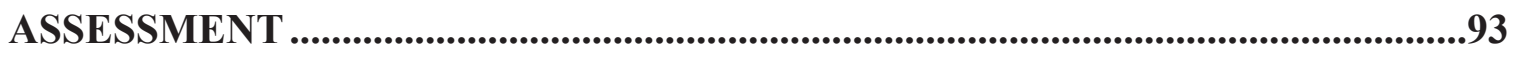

APPENDIX E. EXTENDED METHODOLOGY OF THE CLINICAL STUDY ...102

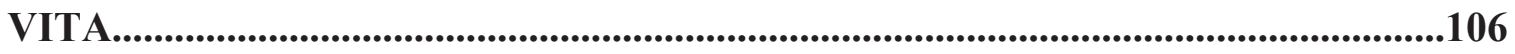




\section{LIST OF TABLES}

Table 3-1. Activated Cylinder Pressures with Corresponding Output Force for Two Pneumatic Cylinders, Computed Transferred Brace Force, and Brace Efficacy

Table 3-2. Native Ankle and Braced Ankle Moment Values at $5^{\circ}$ Increments of Angular Ankle Position

Table 4-1. Peak Plantar Pressures for Each Plantar Area in Each Orthosis Condition During Set Speed Treadmill Walking .....

Table 4-2. Ankle Joint Kinematics in Each Orthosis Condition During Set Speed Treadmill Walking

Table 4-3. $\quad$ Preferred Speed During Over-Ground Walking and Spatio-Temporal Variables in Each Orthosis Condition During Set Speed Treadmill Walking .46

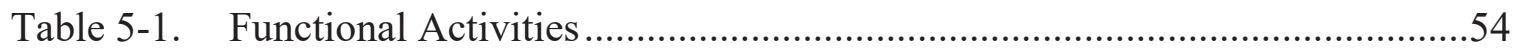

Table 5-2. Patient Demographics and Group Characteristics ....................................57

Table 5-3. Spatio-Temporal Variables of Walking Speed Before, During, and After Testing

Table 5-4. Vertical In-Shoe Force and Spatio-Temporal Variables During LevelWalking and Stair Walking for Control and Dynamic Ankle-Foot Orthosis Conditions

Table 5-5. Distribution of Direction of VAS Pain Score Responses in DAO Relative to CON for Each Activity, and VAS Pain Scores for CON and DAO Conditions for All Participants, "Improved Symptoms," and "No Change in Symptoms".

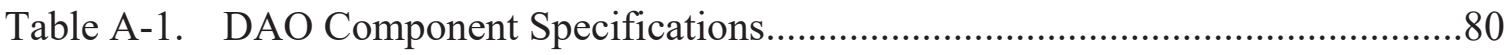

Table A-2. Equipment Specifications

Table B-1. Ankle Angular Kinematics and GRF During Walking in Each Bracing Condition

Table D-1. Preferred Speed During Over-Ground Walking and Spatio-Temporal Variables in Each Orthosis Condition During Set Speed Treadmill Walking 
Table D-2. Ankle Joint Kinematics in Each Orthosis Condition During Set Speed Treadmill Walking for Braced and Unbraced Limbs ....................................99

Table D-3. Knee and Hip Joint Kinematics in Each Orthosis Condition During Set Speed Treadmill Walking for Braced and Unbraced Limbs ........................100 


\section{LIST OF FIGURES}

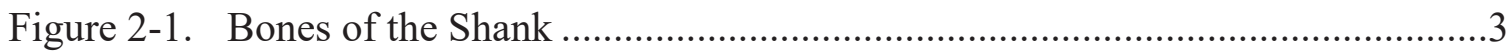

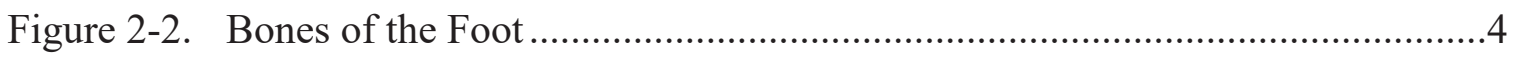

Figure 2-3. Organization of the Bones, Major Joints, and Regions of the Foot and

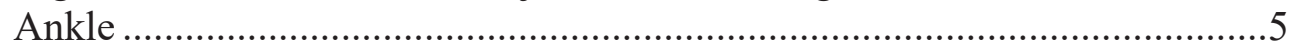

Figure 2-4. Ankle Joint Complex in the Frontal Plane and Transverse Plane...................

Figure 2-5. Average Ankle Compression Force During the Stance Phase of Gait for Healthy Weight and Overweight Individuals .......................................... 8

Figure 2-6. Plantar Pressures During Standing and Walking for Obese Versus NonObese Men and Women ......................................................................... 9

Figure 2-7. Terminology to Describe the Events of the Gait Cycle ..............................11

Figure 2-8. Custom Solid and Articulating Ankle-Foot Orthoses ................................15

Figure 2-9. Ankle Motion in the Sagittal Plane with Various Orthoses .........................17

Figure 2-10. Ankle Motion in the Frontal Plane with Various Orthoses .........................18

Figure 2-11. Diagram of the Offloading Effect in the PTB Cast and AFO .....................20

Figure 2-12. Combined Effect of PTB Design and Heel Clearance on Plantar Offloading

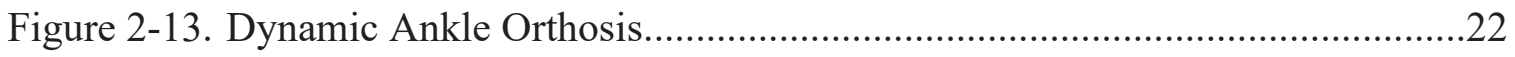

Figure 3-1. Standard of Care Leather and Metal Double Upright Ankle-Foot Orthosis .25

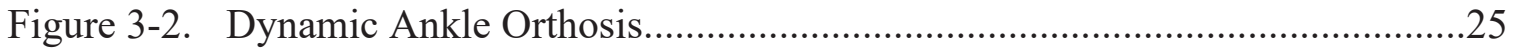

Figure 3-3. Sagittal Plane Range of Motion of the DAO During Weight-Bearing .........26

Figure 3-4. Frontal Plane Range of Motion of the DAO During Weight-Bearing..........26

Figure 3-5. Setup for Characterizing Cylinder Pressure-Output Force Relationship ......28

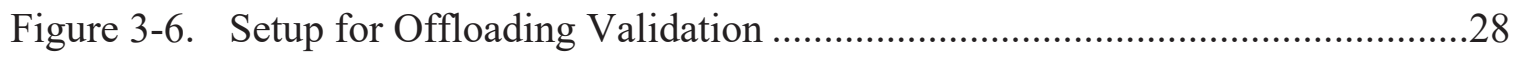

Figure 3-7. Setup for Mobility Validation of DAO Using an Isokinetic Dynamometer .30 
Figure 3-8. Percent of Bodyweight Offloaded by the DAO at Varying Levels of Cylinder Pressure

Figure 3-9. Box Plot of Resistive Ankle Moment Measured at $5^{\circ}$ Increments of Ankle Angular Position for Each Bracing Condition.

Figure 4-1. Orthosis Configurations

Figure 4-2. Sagittal Plane Ankle Joint Angle Ensemble Curves for the Right Limb of All Three Orthosis Conditions

Figure 4-3. Frontal Plane Ankle Joint Angle Ensemble Curves for the Right Limb of All Three Orthosis Conditions

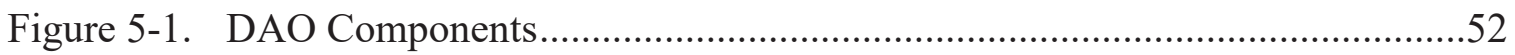

Figure 5-2. Distribution of Diagnoses in the "Improved Symptoms" and "No Change in Symptoms" Groups ...............................................................................58

Figure 6-1. Patent Application for the Dynamic Ankle Orthosis .....................................67

Figure B-1. Bracing Conditions for the Over-Ground Walk Pilot Study..........................84

Figure B-2. Preferred Speed for Each Bracing Condition.................................................86

Figure B-3. Ankle Angles in the Sagittal Plane and Frontal Plane ...................................87

Figure C-1. Equipment and Setup for the Functional Assessment Study .........................88

Figure C-2. Reflective Marker Placement for the Bracing Conditions ..............................89

Figure C-3. Treadmill Walking Activity During the Functional Assessment of the $\mathrm{DAO}$ .90

Figure C-4. Sample Visual3D Model Reconstruction of Motion Capture Data During

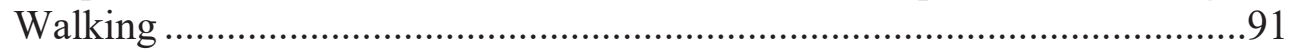

Figure C-5. Sample Plantar Pressure Map for CON and DAO During Walking.............92

Figure D-1. Ankle Joint Kinematic Ensemble Curves in Sagittal Plane and Frontal Plane for the Braced and Unbraced Limbs

Figure D-2. Knee Joint Kinematic Ensemble Curves in Sagittal Plane for the Braced and Unbraced Limbs .95

Figure D-3. Hip Joint Kinematic Ensemble Curves in Sagittal Plane for the Braced and Unbraced Limbs 
Figure E-1. Foot and Ankle Disability Index Score Survey Used to Quantify Disability Level at the Start of the Clinical Study

Figure E-2. Visual Analog Scale Used to Quantify Pain Levels Following the Completion of Functional Activities in the Clinical Study..... 103

Figure E-3. Equipment Used During the Clinical Study................................................104

Figure E-4. Sample Vertical Force Parameters Derived from Insole Data .....................105 


\section{LIST OF ABBREVIATIONS}

\begin{tabular}{|c|c|}
\hline $\mathrm{AFO}$ & Ankle-Foot Orthosis \\
\hline ASCENT & Stair Ascent or Upward Stair Walking \\
\hline ASO & Ankle Stabilizing Orthosis \\
\hline AV & Angular Velocity \\
\hline BMI & Body Mass Index \\
\hline BW & Bodyweight \\
\hline $\mathrm{CON}$ & Control \\
\hline CFR & Code of Federal Regulations \\
\hline CPOP & Chronic Postoperative Pain \\
\hline DAO & Dynamic Ankle Orthosis \\
\hline DECENT & Stair Descent or Downward Stair Walking \\
\hline DF & Dorsiflexion \\
\hline DFM & Distractive Force Mechanism \\
\hline DUAFO & Double Upright Ankle-Foot Orthosis \\
\hline ES & Early Stance \\
\hline EV & Eversion \\
\hline FADI & Foot \& Ankle Disability Index \\
\hline $\mathrm{F}_{\mathrm{b}}$ & Brace Force \\
\hline FDA & United States Food and Drug Administration \\
\hline $\mathrm{F}_{\text {LLC }}$ & Left Load Cell Force \\
\hline $\mathrm{F}_{\mathrm{RLC}}$ & Right Load Cell Force \\
\hline FS & Foot Strike \\
\hline GRF & Ground Reaction Force \\
\hline $\mathrm{H}_{\mathrm{A}}$ & Alternative Hypothesis \\
\hline HFO-A & Articulating Hindfoot Orthosis \\
\hline HFO-R & Rigid Hindfoot Orthosis \\
\hline $\mathrm{Ho}_{\mathrm{O}}$ & Null Hypothesis \\
\hline LSD & Least Significant Difference \\
\hline MCID & Minimum Clinically Important Difference \\
\hline $\mathrm{NIH}$ & National Institutes of Health \\
\hline $\mathrm{OA}$ & Osteoarthritis \\
\hline OGWALK & Over-ground Walking \\
\hline $\mathrm{PF}$ & Plantarflexion \\
\hline PP & Peak Plantar Pressure \\
\hline PTB & Patellar Tendon Bearing Cast or Orthosis \\
\hline PTOA & Post-Traumatic Ankle Osteoarthritis \\
\hline PTTD & Posterior Tibialis Tendon Dysfunction \\
\hline RA & Rotational Axis \\
\hline ROM & Range of Motion \\
\hline SD & Standard Deviation \\
\hline TMWALK & Treadmill Walking \\
\hline VAS & Visual Analog Scale \\
\hline $3 \mathrm{D}$ & Three Dimensional \\
\hline
\end{tabular}




\section{CHAPTER 1. INTRODUCTION}

Over two million Americans visit the doctor each year for foot and ankle pain due to a degenerative condition or injury.[1] Prevalence estimates for foot and ankle pain vary from $9 \%$ to $36 \%$ due to inconsistency in how "foot and ankle pain" is defined.[2] Even though it is associated with female sex, obesity, and older age, foot and ankle pain is known to impact individuals of all ages and stages of life.[2] When left unchecked, foot and ankle pain is a known risk factor for loss of independence, locomotor disability, impaired balance, and increased risk of falling. [2-7]. Lower limb joint pain can also have a significant detrimental impact on health-related quality of life due to reduced functional abilities (i.e., walking, being active, etc.).[6,8-13]

Orthopedic foot and ankle conditions such as ankle sprains, osteoarthritis, stress fractures, traumatic injuries, tendonitis, synovitis and deformities can often present symptoms of acute or chronic pain, aching, and joint stiffness. Common treatment options include surgical procedures, prescription medication, physical therapy, and conservative bracing treatment (i.e., orthosis, orthotic, etc.). These treatment options aim to reduce pain, maintain function, improve range of motion, accommodate an existing anatomical deformity, and prevent further deformity. Conservative treatment options such as modified footwear, orthotics, or an Ankle-Foot Orthosis (AFO) and Ankle Stabilizing Orthosis (ASO) can effectively manage pain symptoms of lower limb conditions,[14-16] but they can also significantly hinder joint motion.[17,18] The restrictive nature of current practice devices results in decreased functional capacity and an altered gait which may lead to other issues, disease progression, and eventually, surgery.[17,18]

The Dynamic Ankle Orthosis (DAO) was designed to manage mechanical pain (i.e., weight-bearing-evoked or movement-evoked) and to restore function to individuals suffering from orthopedic foot and ankle conditions. The DAO utilizes an offloading mechanism to alleviate lower limb pain without interfering with natural joint motion. This dissertation describes the development, validation, and implementation of this novel treatment option. The organization of this dissertation is as follows: Chapter 2 presents background information and literature review to describe the motivation for this work. Chapter 3 details the development and design validation of the DAO. Chapter 4 details a functional gait assessment performed with ten healthy individuals comparing the DAO to a standard of care AFO and an unbraced control. Chapter 5 details a clinical study performed with twenty-five patients to evaluate the effect of DAO wear on pain symptoms, underfoot loading, and the ability to complete functional tasks. Chapter 6 provides an overview of the path to commercialization for this new device, some considerations for future directions with this device, and brief concluding remarks. 


\section{CHAPTER 2. BACKGROUND}

This chapter provides the background material necessary to understand the content of this dissertation. In particular, this chapter describes relevant concepts and provides a brief literature review to establish the foundation for this collection of work.

\section{Anatomy of the Lower Leg}

To understand the principles of biomechanics and clinical concepts that are described in this dissertation, a basic understanding of anatomical nomenclature is vital. When relating parts of the body, relative descriptors are often used: superior (towards the head), inferior (towards the feet), anterior (towards the front of the body), posterior (towards the back of the body), medial (closer to the midline of the structure or body), lateral (farther from the midline of the structure or body), proximal (closer to the trunk or point of origin), distal (farther from the trunk or point of origin), superficial (closer to the surface), and deep (farther from the surface). The human body has three anatomic planes that transect the body and are used to describe the direction of movements: the sagittal plane (vertical plane running anterior to posterior), frontal plane (vertical plane running medial to lateral), and transverse plane (horizontal plane). Segmental motion of the body occurs when muscles contract and act across joints, the places where two or more bones come together, similar to a lever with a fulcrum. Static stability of a joint is provided by bones and ligaments, and dynamic joint stability is provided by muscles and tendons. Ligaments and tendons are both types of fibrous connective tissue that either connect bone to bone (ligaments) or muscle to bone (tendons).

The portion of the lower leg between the knee and ankle joints is called the "shank" segment and is comprised of two long bones, the tibia and fibula (Figure 2-1). The tibia is the larger of the two and is located toward the inside of the lower leg, medially. It is the primary weight-bearing bone of the lower leg. The fibula is the smaller bone and is located on the outside of the lower leg, laterally. The fibula carries a small portion of bodyweight and acts as a point of fixation for muscle attachments. The tibia and fibula are firmly held together proximally and distally with ligaments. Distally, the tibia and fibula are held together by a fibrous joint known as the syndesmosis which plays a very important role in ankle joint stability. Below the ankle joint is the foot segment, which contains twenty-six individual bones that are supported by a complex network of muscles, tendons, and ligaments, resulting in over thirty articulating joints in total (Figure 2-2). The foot is traditionally divided into the hindfoot, midfoot, and forefoot regions. The hindfoot contains the two largest tarsal bones, the talus and calcaneus, and is separated from the midfoot by the transverse tarsal joint (Figure 2-3). The remaining tarsal bones, the navicular, cuboid, and three cuneiforms, form a geometric arch in the midfoot, which is a critical component for force absorption and healthy foot mechanics. The tarsometatarsal joint separates the midfoot from the forefoot, which contains the five metatarsal bones and fourteen phalangeal bones. 


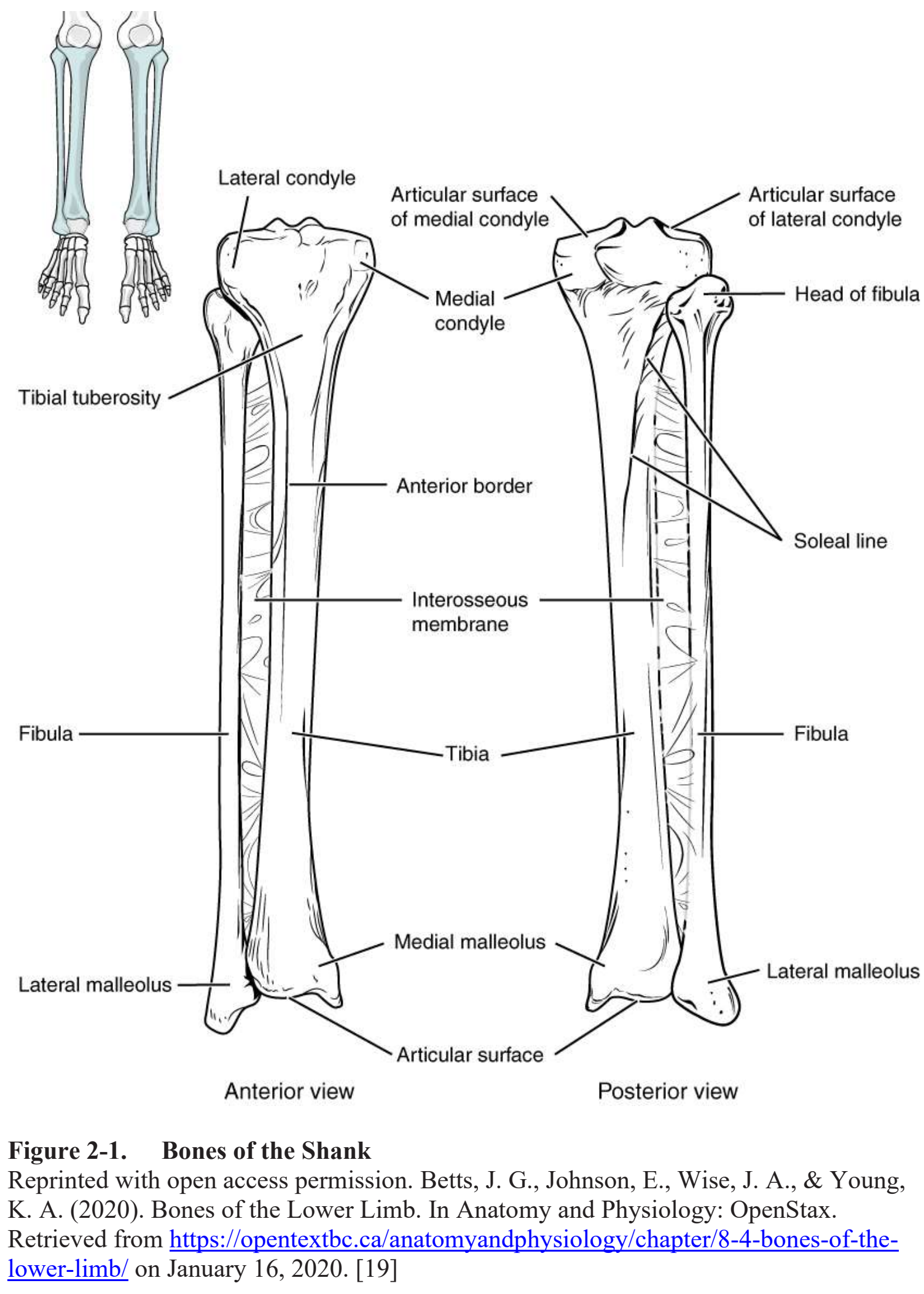



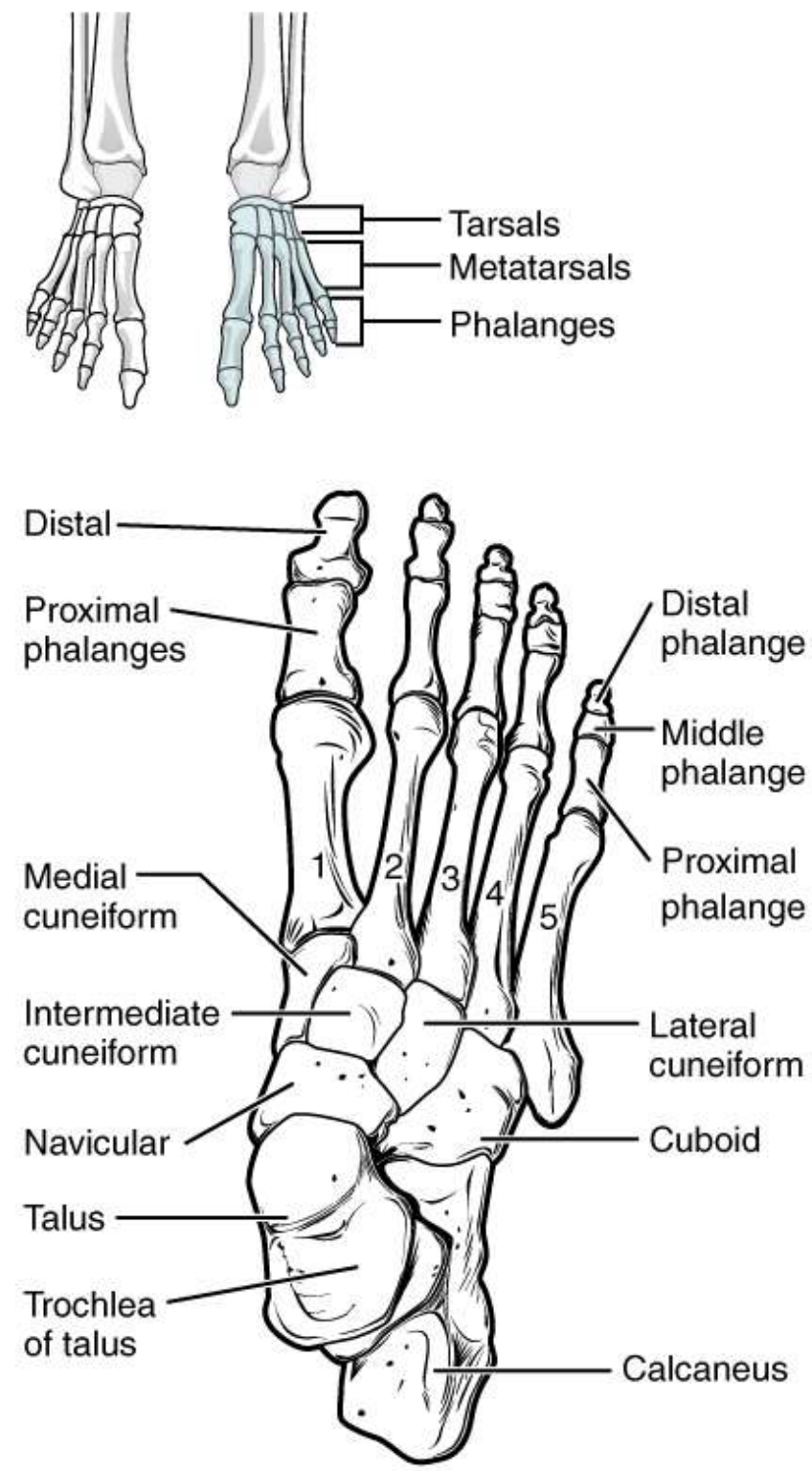

Superior view

Figure 2-2. Bones of the Foot

Reprinted with open access permission. Betts, J. G., Johnson, E., Wise, J. A., \& Young, K. A. (2020). Bones of the Lower Limb. In Anatomy and Physiology: OpenStax.

Retrieved from https://opentextbc.ca/anatomyandphysiology/chapter/8-4-bones-of-thelower-limb/ on January 16, 2020. [19] 


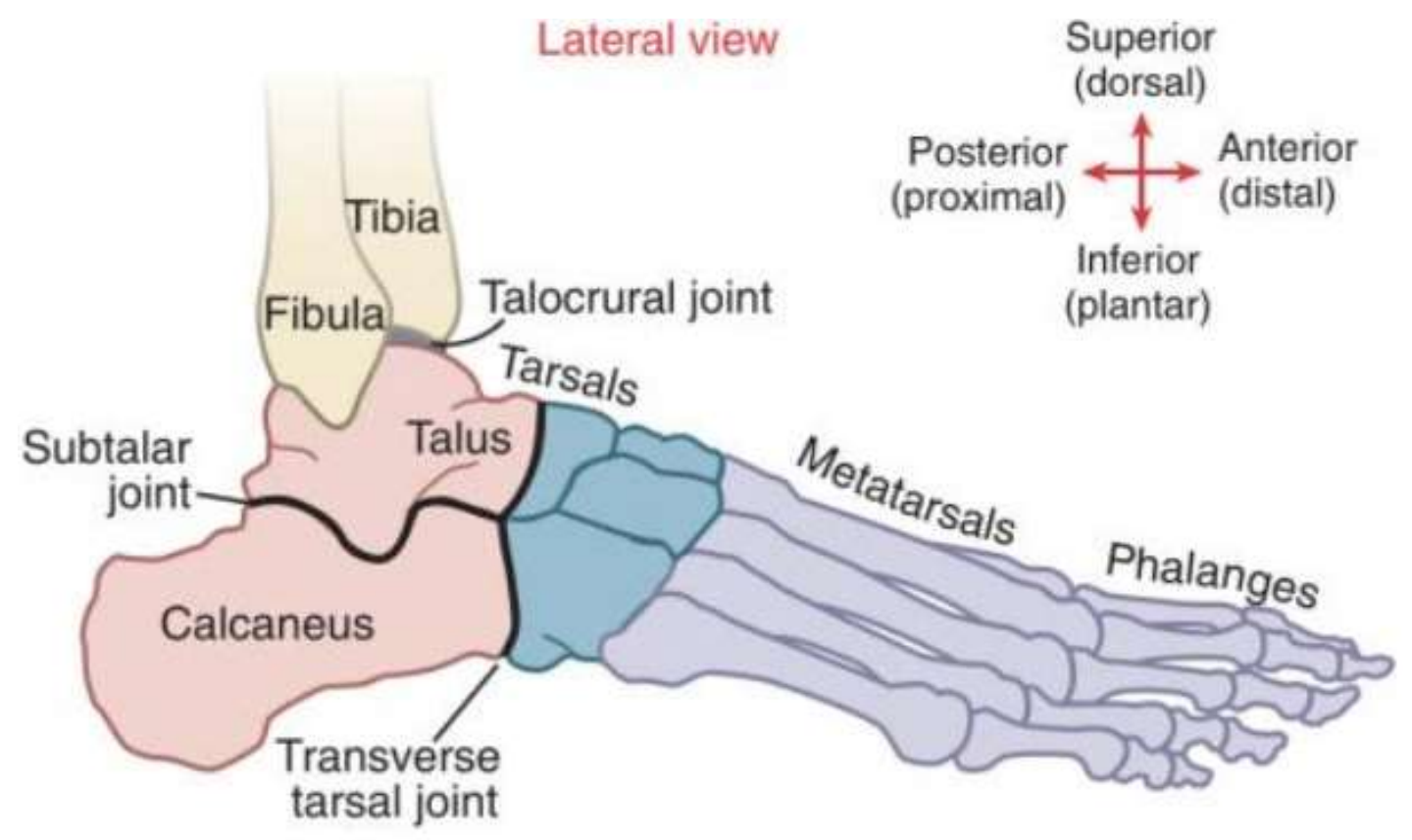

\section{Rearfoot Midfoot Forefoot}

Figure 2-3. Organization of the Bones, Major Joints, and Regions of the Foot and Ankle

Reprinted with permission. Neumann, D. (2010). "Ankle and Foot" and "Kinesiology of Walking”. In Kinesiology of the Musculoskeletal System: Foundations of Physical

Rehabilitation (2nd ed., pp. 574-580, pp.636): Mosby. ISBN-13: 978-0323039895 [20] 
The point where the bones of the shank and foot come together is called the ankle joint (Figure 2-4). The tibia, fibula, and talus come together to make the upper compartment of the ankle joint, known as the talocrural joint. The distal tibia is aligned directly over the talus and extends over the medial side, forming a bony prominence called the medial malleolus. The distal fibula completes the bony arch, or what is called the ankle mortise, adding significant stability to the ankle joint and forming another bony prominence called the lateral malleolus. Together, the medial and lateral malleolus can be used to palpate and locate the talocrural joint axis. This axis is naturally oblique, offset about $10^{\circ}$ in the frontal plane and $13-18^{\circ}$ in the transverse plane.[20]

Acting as a sagittal plane hinge-type joint, the talocrural joint allows the foot to be moved into dorsiflexion (toes pointing upwards) and plantarflexion (toes pointing downwards). The lower compartment of the ankle joint, called the subtalar joint, is formed by the articulation of the inferior surface of the talus on the superior surface of the calcaneus. The subtalar joint works together with the transverse tarsal joint to permit complex tri-planar movement around an oblique axis, chiefly frontal plane inversion (inward turning of the foot) and eversion (outward turning of the foot) with some rotational contributions from the sagittal and transverse planes. The functional range of motion of the ankle joint complex varies significantly between individuals. On average, during walking is about $30^{\circ}$ in the sagittal plane ( $10^{\circ}$ dorsiflexion, $20^{\circ}$ plantarflexion) and $15^{\circ}$ in the frontal plane (inversion and eversion). $[13,21]$

Unlike other joints of the lower extremity, the ankle joint has a high congruency with surface area contact ranging from 1.5 to $9.4 \mathrm{~cm}^{2}$.[22] The ankle joint complex bears some of the highest loads in the body. During stance phase of walking, the ankle supports peak loads exceeding 4-5x bodyweight.[13,22] In overweight populations, these compressive loads at the ankle can be increased by 40-48\% (Figure 2-5).[23] Likewise, obese individuals experience significantly higher peak pressures across the plantar aspect of the foot during standing and walking compared to healthy individuals (Figure 2-6).[24] Long-term, repetitive loading with elevated plantar pressures and compressive joint loads can have serious consequences for the anatomical structures of the lower leg.

The stability of the foot and ankle is sustained during weight-bearing activities by a complex network of anatomical structures. Both the talocrural and subtalar joints, as well as many of the joints of the foot, are diarthrotic synovial joints. This means that a joint cavity filled with lubricating synovial fluid exists in the space between the bones, which are coated in articular cartilage. The joint cavity is encapsulated by a synovial membrane, a dense fibrous joint capsule, and various ligamentous structures. Muscles, tendons, nerves, and vessels wrap around the joint capsule externally. The lower leg has four individual muscular compartments that cross the ankle joint and insert on the bones of the foot to extrinsically move the foot. Tendons from these muscles pass through lubricated tendon sheaths to reduce friction as they slide around the joint as the muscles contract. Tendons and other structures are often secured near joints by connective tissue called a retinaculum to prevent "bow-stringing" as the joint angle closes. Disruption to any of these anatomical or joint structures can result in painful, debilitating conditions and severe motion deficits. 


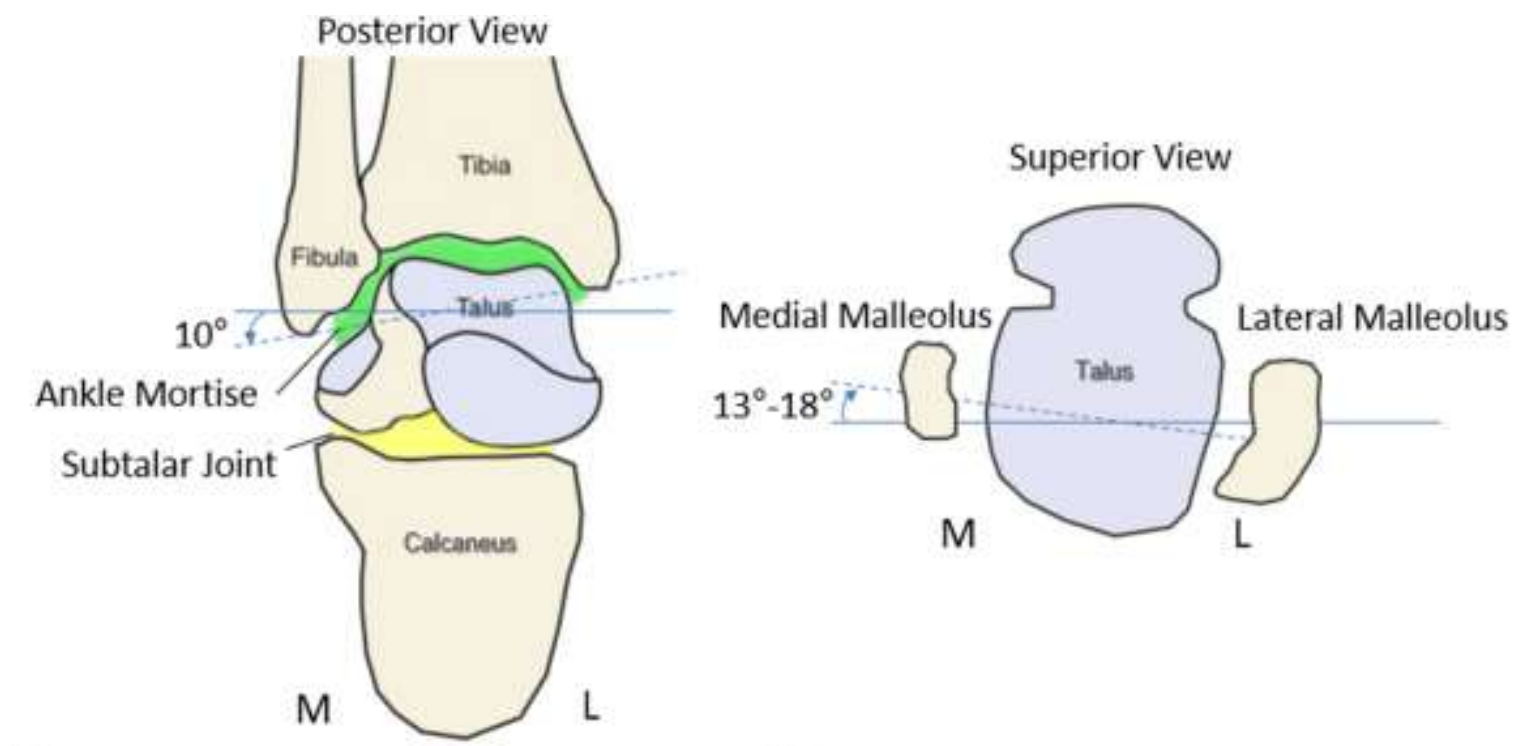

A)

B)

Figure 2-4. Ankle Joint Complex in the Frontal Plane and Transverse Plane A) Frontal Plane and B) Transverse Plane. Reprinted with permission. Neumann, D. (2010). "Ankle and Foot" and "Kinesiology of Walking". In Kinesiology of the Musculoskeletal System: Foundations of Physical Rehabilitation (2nd ed., pp. 574-580, pp.636): Mosby. ISBN-13: 978-0323039895 [20] 


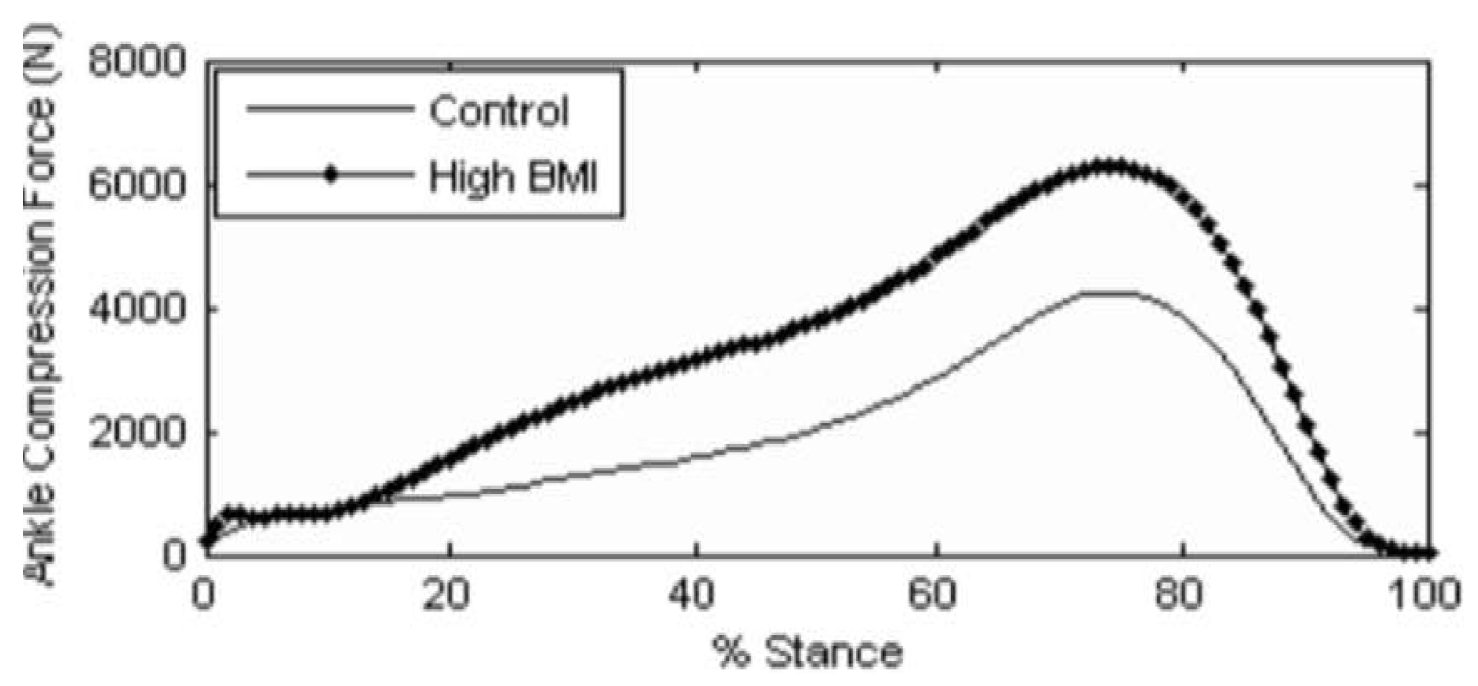

Figure 2-5. Average Ankle Compression Force During the Stance Phase of Gait for Healthy Weight and Overweight Individuals

Healthy Weight (Control) and Overweight (High BMI). Reprinted with permission. Sanford, B., Williams, J., Zucker-Levin, A., \& Mihalko, W. (2014). Hip, Knee, and Ankle Joint Forces in Healthy Weight, Overweight, and Obese Individuals During Walking. In: Computational Biomechanics for Medicine (pp. 101-111). Doyle B., Miller K., Wittek A., Nielsen P. (eds). Springer, New York, NY. https://doi.org/10.1007/978-14939-0745-8 [23] 

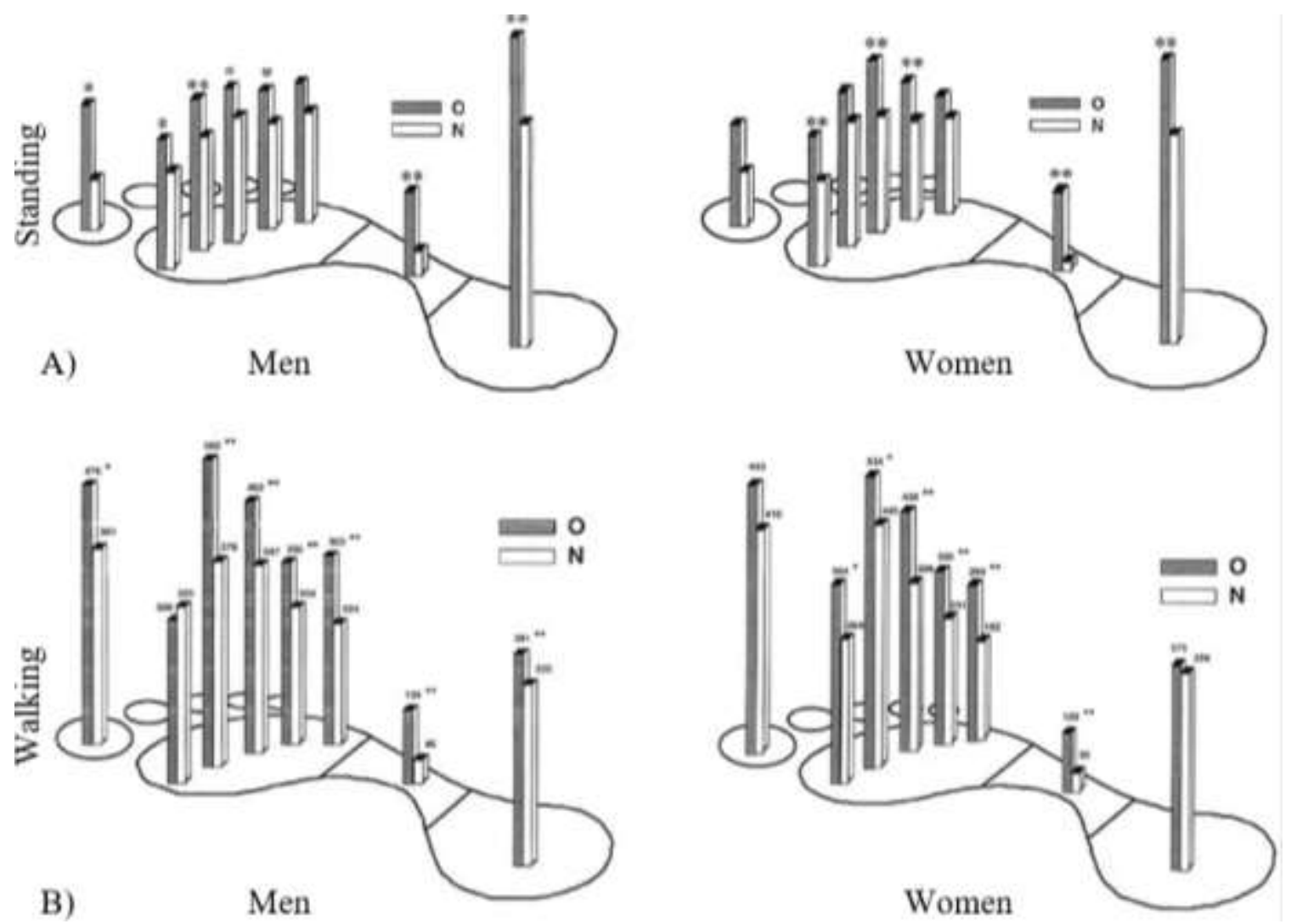

Figure 2-6. Plantar Pressures During Standing and Walking for Obese Versus Non-Obese Men and Women

Plantar Pressures reported in KPa. A) Standing and B) Walking; Obese (O) and NonObese $(\mathrm{N})$; * denotes $\mathrm{p}<0.05$ and $* *$ denotes $\mathrm{p}<0.01$. Reprinted with permission. Hills, A. P., Hennig, E. M., McDonald, M., \& Bar-Or, O. (2001). Plantar pressure differences between obese and non-obese adults: a biomechanical analysis. International Journal of Obesity, 25(11), 1674-1679. https://doi.org/10.1038/sj.ijo.0801785 [24] 


\section{Normal Human Locomotion}

In this dissertation, human walking biomechanics are analyzed and discussed, therefore it is important to understand the phases, subdivisions, and important time points during the gait cycle. Human locomotion is the energy-optimized, bipedal forward propulsion of the body through space.[25] Studying human locomotion requires breaking this complex activity down into the cyclic components of gait. The gait cycle (Figure 2-7) is made up of two primary phases: stance phase (where the foot is in contact with the ground) and swing phase (where the foot is off the ground). Stance phase makes up about $60 \%$ of a single stride and swing phase makes up the other $40 \%$.

Stance phase begins with a period of double limb support, where both feet are in contact with the ground, followed by a period of single limb support, and ending with a second period of double limb support. During the first period of double support, the foot contacts the ground at a point called heel strike or "initial contact." After the heel makes contact, the foot and shank pivot about the heel to a flat or level foot position (also called the heel rocker movement). Next, a period of weight acceptance or the "loading response" occurs where the limb begins to stabilize, shock from the impact is absorbed, and forward progression of the body is preserved. The contralateral limb enters swing phase as the single support phase begins. "Midstance" occurs while the shank continues to rotate forward over the foot, this time pivoting at the ankle (also called the ankle rocker movement). As a result, the center of mass of the body to progresses over the foot like an inverted pendulum.[26]

Following midstance is "terminal stance," where momentum continues to advance the center of mass in front of the support foot and the heel raises off the ground as the foot pivots (also called the forefoot rocker movement). As the contralateral limb returns from swing phase and contacts the ground, the stance limb enters a second, brief double support phase called "pre-swing." The foot continues to rise and eventually the toes leave the ground at a point called toe-off or push off, propelling the center of mass forward. The swing phase is divided into three sub-phases: initial swing (acceleration), mid-swing, and terminal swing (deceleration).

Although it may seem like a simple task, walking is only made possible through the rhythmic coordination of specialized physiological systems.[27] During the gait cycle, the muscles of the lower extremity progress through a sequence of inactivity, eccentric contractions (muscle active and lengthening), and concentric contractions (muscle active and shortening). The effect of this strategic muscular activity is that the leg can absorb shock from impact (i.e., the sudden deceleration of the foot that occurs at initial contact), stabilize, lift the foot, accelerate, control the foot's position, and decelerate the limb with minimal displacement of the body's center of mass and, therefore, maximize energy conservation. This careful synchronization, however, can be disrupted by many external and internal factors, resulting in abnormal gait patterns. 


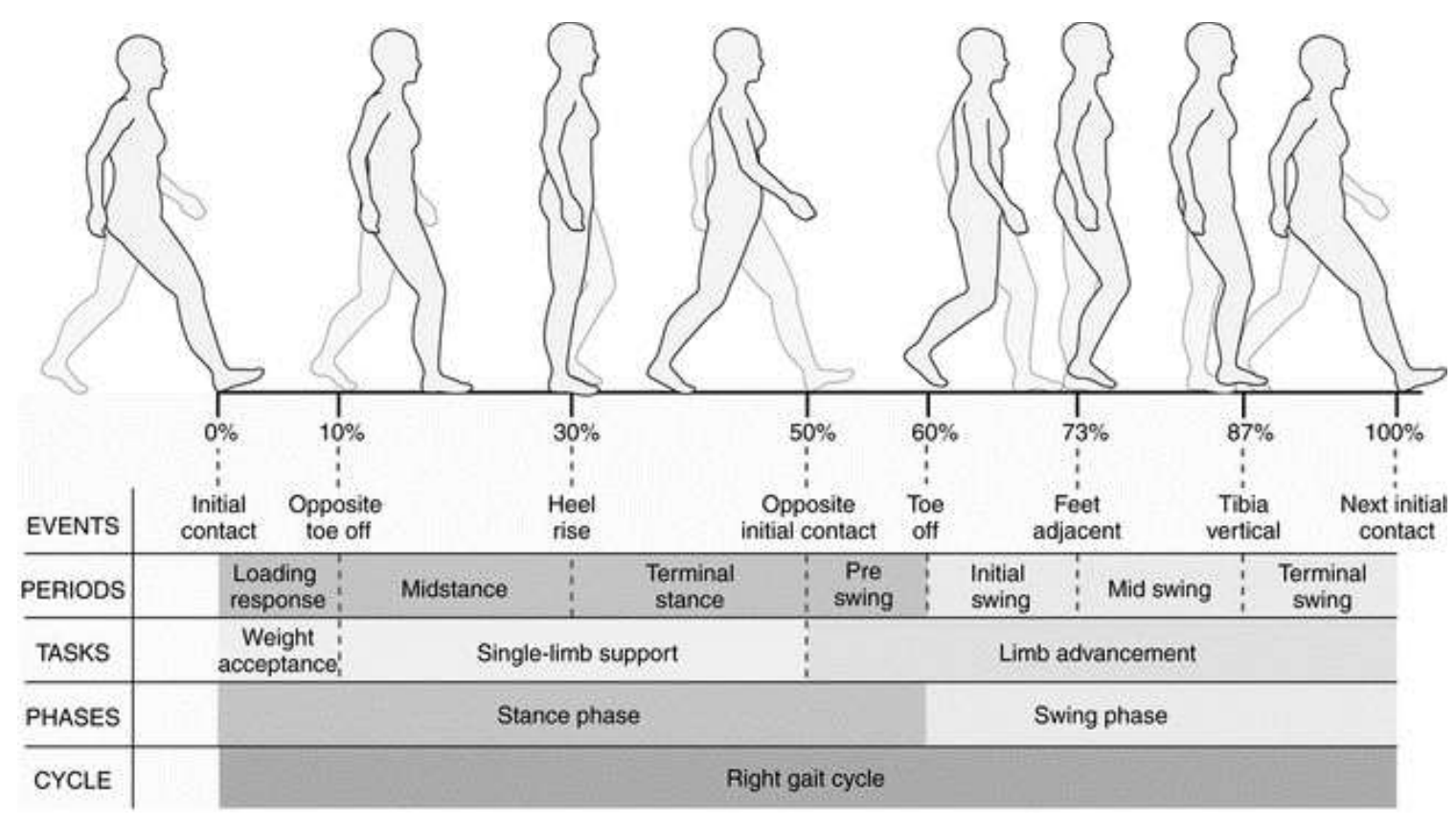

Figure 2-7. Terminology to Describe the Events of the Gait Cycle

Reprinted with permission. Neumann, D. (2010). "Ankle and Foot" and "Kinesiology of Walking”. In Kinesiology of the Musculoskeletal System: Foundations of Physical

Rehabilitation (2nd ed., pp. 574-580, pp.636): Mosby. ISBN-13: 978-0323039895 [20] 


\section{Conditions of the Lower Leg that Present Pain and Impact Function}

The lower leg and foot are responsible for providing support and stability during weight-bearing activities such as standing, walking, running, and jumping. When exceedingly high stresses are absorbed by the anatomical structures of the lower leg, structural failures can occur and develop into life-long debilitating conditions. Examples of relevant conditions are described as follows.

\section{Acute Injuries}

Acute foot and ankle injuries including sprains/strains, contusions, and fractures account for over $20 \%$ of emergency room visits in the United States annually, with teenagers and young adults ( $<45$ years old) presenting the highest incidence rates.[28] An ankle sprain occurs when the foot is planted unevenly and twisted beyond its normal range of motion. Ankle sprains are one of the most common musculoskeletal injuries in athletes, military, and the general population.[29-32] Lateral (inversion) ankle sprains are most common. On the lateral aspect of the ankle is a complex of three ligaments that provides stability by attaching the lateral malleolus to the talus and calcaneus. Medial (eversion) ankle sprains are less common because the medial aspect of the ankle is stabilized by a wide, thick complex ligament known as the deltoid ligament. When a mild ankle sprain (grade I) occurs, a ligament is stretched, and a severe ankle sprain (grade III) is when a ligament is completely torn. Grade III sprains are often treated with a cast, brace, and surgery in some cases, and they can result in permanent instability of the joint.[32]

Ankle fractures represent a significant portion of orthopedic trauma injuries that occur in the United States. In 2014, over 60,000 work-related ankle injuries were reported to the U.S. Department of Labor with average costs up to $\$ 5,900$ per injury.[33,34] Ankle fractures commonly include malleolar, bimalleolar, trimalleolar and pilon fractures. With an ankle fracture, the patient will present symptoms of pain, swelling, and motion deficits. Traumatic injuries such as fractures are the principal cause of developing longterm degenerative orthopedic conditions of the foot and ankle, typically presenting in adults ( $>55$ years old) as joint stiffness, inflammation, swelling, reduced range of motion, and pain exacerbated with increased activity. $[8,9,14]$ One in five middle-aged adults $(\geq 45$ years old) reports general foot and ankle pain, with two-thirds reporting moderate or worsening impairment with daily activities.[7,35] Older adults ( $\geq 65$ years old) are affected by foot and ankle pain the most, with $30.9 \%$ experiencing acute tenderness of the foot and ankle and 14.9\% report reoccurring pain of the foot and ankle.[36]

\section{Degenerative Conditions}

Osteoarthritis. Patients who sustain irreversible cartilage damage or joint instability from ankle injuries are likely to develop ankle arthritis. Affecting approximately $1 \%$ of adults, ankle osteoarthritis (OA) is a degenerative disease that 
occurs when the cartilage layer in the joint wears away over time.[8] Unlike OA of other joints, $70 \%-80 \%$ of ankle OA cases are associated with a previous traumatic injury, a condition known as Post-Traumatic Ankle Osteoarthritis (PTOA).[8,9] Long-term physical impairments, joint pain, and reduced quality of life are all possible outcomes of this debilitating disease. The main indicators of ankle OA are progressive joint pain, inflammation of the joint, and substantial decrease of overall range of motion and stability of the joint. The mechanisms for pain associated with OA are reduced cartilage, narrowed joint space, and the development of painful osteophytes where excess articular surface contact occurs during weight-bearing.

Posterior Tibialis Tendon Dysfunction. The posterior tibialis muscle is located in the deep calf of the lower leg. This muscle is responsible for inversion and plantarflexion of the foot as well as stabilization of the arch of the foot. The tendon for this muscle passes the ankle joint on the medial side, wrapping behind the medial malleolus in what is known as the tarsal tunnel. The posterior tibialis tendon can become inflamed, stretched, or torn due to acute injuries or over-use. The tendon may not be able to provide joint stability and support for the arch of the foot, resulting in flatfoot (fallen arch) and poor ankle alignment. This degenerative disease is known as posterior tibialis tendon dysfunction (PTTD). Pain and swelling in and around the ankle are often present in addition to substantial loss of function in the later stages of this disease.

Chronic Postoperative Pain. General postoperative pain is a common adverse outcome with orthopedic surgeries. In most cases, this acute pain is managed effectively and resolves in a short period. Chronic postoperative pain (CPOP) is persistent postsurgical pain that lasts months or years after the surgical site is healed.[37,38] Incidence of CPOP is especially high following orthopedic procedures: $29 \%$ after fracture repair, $16 \%$ after OA procedure, $32.7 \%$ after joint arthroscopy. [37,39] CPOP can have a variety of mechanisms (i.e., movement-evoked, temperature sensitivity, neuropathic, multimodal, etc.) and is extremely difficult to treat. $[38,40]$

Plantar Fasciitis. Just under the skin of the bottom (plantar aspect) of the foot, a fibrous band of connective tissue called the plantar fascia connects the base of the toes to the heel. This band protects the structures of the sole of the foot from injury, dynamically stabilizes the structures of the foot, and lifts the arch of the foot. The plantar fascia is cyclically loaded and stretched during ambulation. Over-use and poor foot mechanics can cause the fascia to become irritated, inflamed, and begin to fray with micro-tears. Plantar fasciitis presents as a stabbing pain localized near the heel, often worse after a long rest.

Nerve Disorders such as Morton's neuroma involves damaged or thickened nerve tissue on the plantar aspect of the foot due to irritation of the nerve by a mechanical factor such as repetitive compression.[41] Morton's neuroma presents as an enlarged nerve that usually presents in the space between the third and fourth toes, and is symptomatic with localized pain in the interspace. Anything including poor shoes, foot deformities, overuse, and co-morbidities can result in the development of a hypersensitive nerve disorder. 


\section{Current Treatment Options}

Current treatment options aim to reduce pain, maintain function, improve range of motion, accommodate an existing anatomical deformity, and prevent further deformity. Common treatment options include surgical procedures, prescription medication, physical therapy, and conservative bracing treatment (including modified footwear, orthotics, rocker-bottom stabilizing boots, and orthoses). In cases of severe joint pain, surgical treatment such as an ankle replacement (arthroplasty) or ankle fusion (arthrodesis) may be the best option. Over 80,000 ankle fusions and 16,000 ankle replacements are performed annually, creating a significant economic burden of $\$ 11$ billion in the Medicare population.[33,34,42,43] However, surgical procedures are usually only considered after conservative treatment options are unsuccessful. In fact, for cases of mild to moderate joint pain, orthoses have been shown to be quite successful at managing pain symptoms of the foot and ankle.[14-16]

\section{Orthosis Design}

An Ankle-Foot Orthosis (AFO) is an external apparatus that is intended to be worn around the segment, either within the shoe or in place of the regular shoe. Orthoses are available as prefabricated, off-the-shelf products as well as custom fabricated devices. Custom orthoses are available with soft, semi-rigid, and rigid constructs depending on the user's needs, with semi-rigid orthoses being the most common. Semi-rigid orthoses offer a combination of advantages including cushioning, shock absorption, and protection with weight redistribution, support, and control for painful conditions and flexible deformities.[44] Custom orthoses (Figure 2-8) can be fabricated with solid joints and locked ankle position to prevent uncontrolled plantarflexion (i.e., in cases of foot drop due to weak dorsiflexors) or toe drag during walking. Solid orthoses are often comprised of lightweight plastic material lined with a layer of cushioning that has been custom molded to fit the patient and maintain a desired ankle position. Orthoses can also be designed with articulating pin joints to provide either free or controlled sagittal plane ankle motion. By incorporating a feature that provides a physical plantarflexion stop, plantarflexion resistance, or dorsiflexion assistance (posterior leaf spring), the brace can prevent excessive plantarflexion while permitting natural progression of the tibia over the foot and natural dorsiflexion of the foot during the stance phase of walking. Articulating orthoses can be made with plastic materials or using metal and leather components adapted to the user's existing footwear. Ultimately, the biomechanical function and design of the prescribed orthosis must match the needs of the patient.[45]

\section{Orthosis Mechanics}

The goals of treatment with an orthosis are to restore normal function as well as to prevent further progression of the disease process affecting the foot and ankle.[46] To achieve these goals, orthoses are designed to absorb and attenuate shock, dissipate loads by reducing or offloading high-pressure areas, minimize shear forces, provide cushion to 

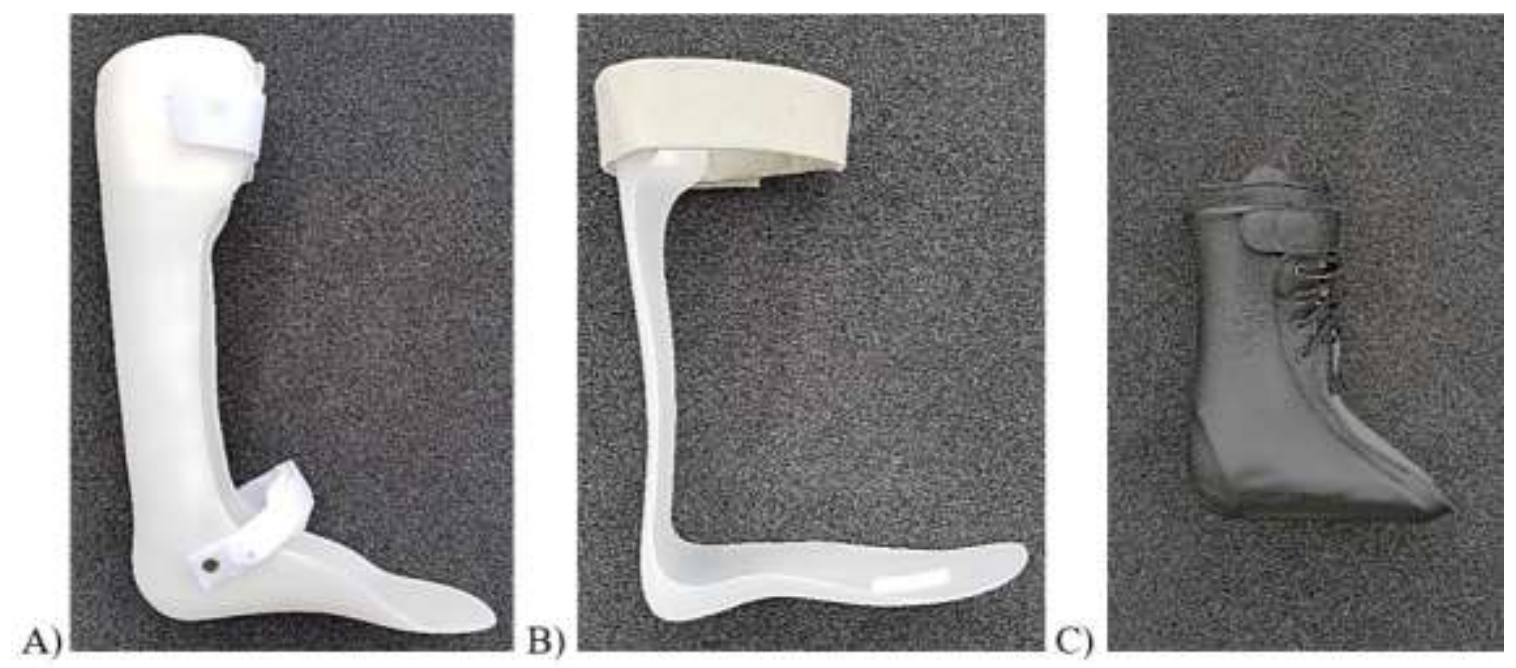

D)
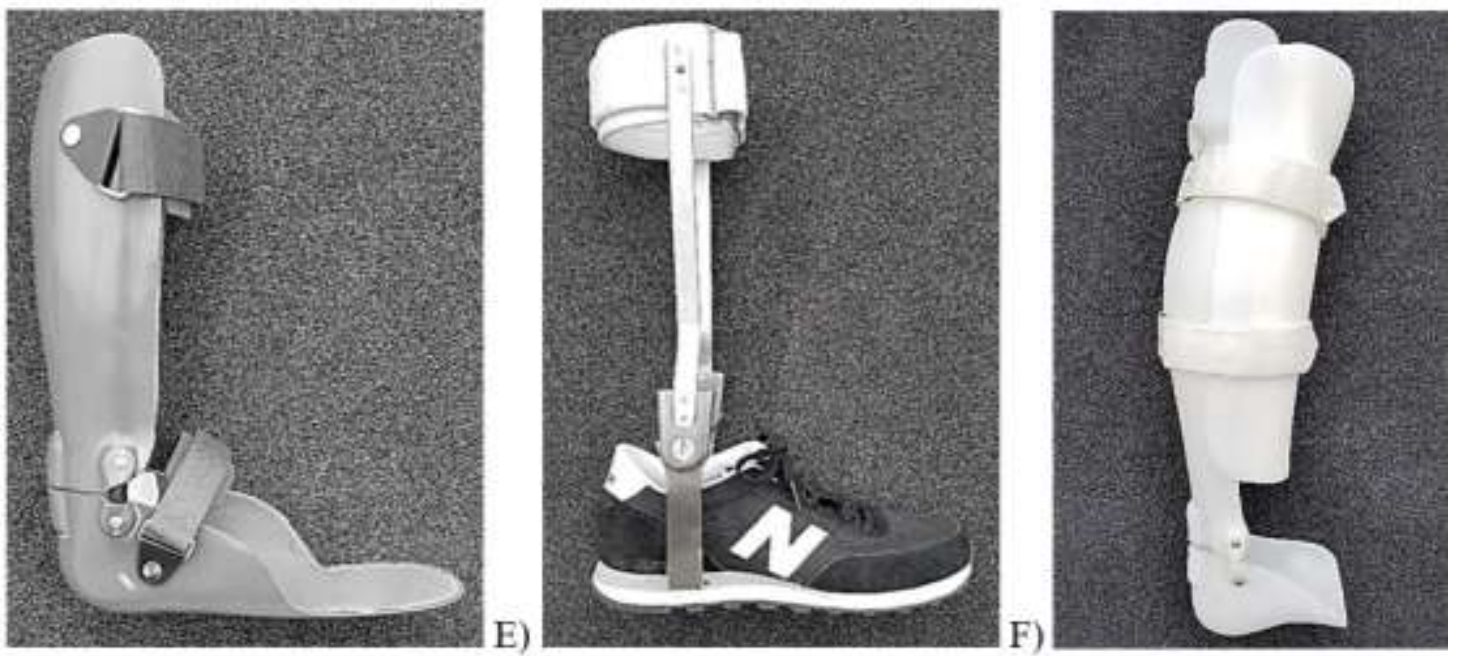

Figure 2-8. Custom Solid and Articulating Ankle-Foot Orthoses

A) Solid AFO, B) Posterior Leaf Spring AFO, C) Leather Lacer AFO, D) Articulating AFO, E) Conventional Leather and Metal Double Upright AFO, and F) Patellar Tendon Bearing AFO. 
tender areas, or correct flexible deformities while improving joint alignment and providing joint control and support.[46] These desired effects are achieved by applying forces in specific places on the segment to redistribute or transfer pressures as well as control motion. A standard AFO offers joint stability as the primary mechanism for pain relief. Specifically, ankle-foot orthoses reduce pain of degenerative hindfoot conditions by maintaining subtalar alignment and limiting ankle motion during gait (Figures 2-9 and 2-10).[17,46]

We believe that simply bracing the foot and ankle with a traditional orthosis falls short of fully addressing the issue at hand, which is that the individual has lost function of the foot and ankle due to pain and is impaired as a result. By limiting motion at the ankle with a traditional AFO, the patient exchanges their ability to walk normally for the benefit of pain relief.[17,18] This altered gait due to the restrictive nature of existing braces may lead to disease progression and the need for surgery. As such, an alternative treatment strategy is needed for the treatment of painful conditions of the foot and ankle, specifically one that can provide pain relief without compromising joint motion.

\section{Alternative Treatment Strategy}

It is well-known that general weight loss has many health, lifestyle, and social benefits. The NIH guidelines recommend that overweight and obese individuals strive for at least $10 \%$ weight loss as the first step towards managing their condition. [47] Under the NIH Osteoarthritis Initiative, researchers observed that 5-10\% weight loss slows the progression of cartilage degeneration in cases of knee OA with lower rates of progression associated with greater weight loss.[48] Not only does weight loss have a clear doseresponse effect, but a $10-20 \%$ reduction in bodyweight has also been shown to have substantial clinical and mechanistic benefits in terms of pain, function, and quality of life for individuals with degenerative conditions.[49-52]

Applying this "weight loss" mechanism at the joint level for reduction of acute and chronic pain can be achieved by altering the longitudinal loads passing through the joint ("offload" or "unload" the joint) with an external device. Existing rehabilitation techniques such as hydrotherapy and unloading treadmills have been used to reduce lower extremity loading and improve function, but these options can be difficult to access.[53-58] The "Knee Unloader" orthosis is an existing conservative treatment option for individuals with varus knee OA that improves both pain and functional ability by using a counter-moment (valgus) to improve knee alignment and reduce unicompartmental knee joint contact forces.[59-61] Another knee orthosis utilizes an extension assistance mechanism to reduce tricompartmental knee joint contact forces.[62]

For the foot and ankle, however, the treatment options that utilize an offloading strategy are very uncommon. One such option is a surgical technique called ankle arthrodiastasis.[63] In this procedure, an external fixator device applies traction to reduce the natural compression of the foot and ankle joints.[14,63-66] This technique has been used by physicians to treat degenerative ankle conditions such as ankle arthritis for over 


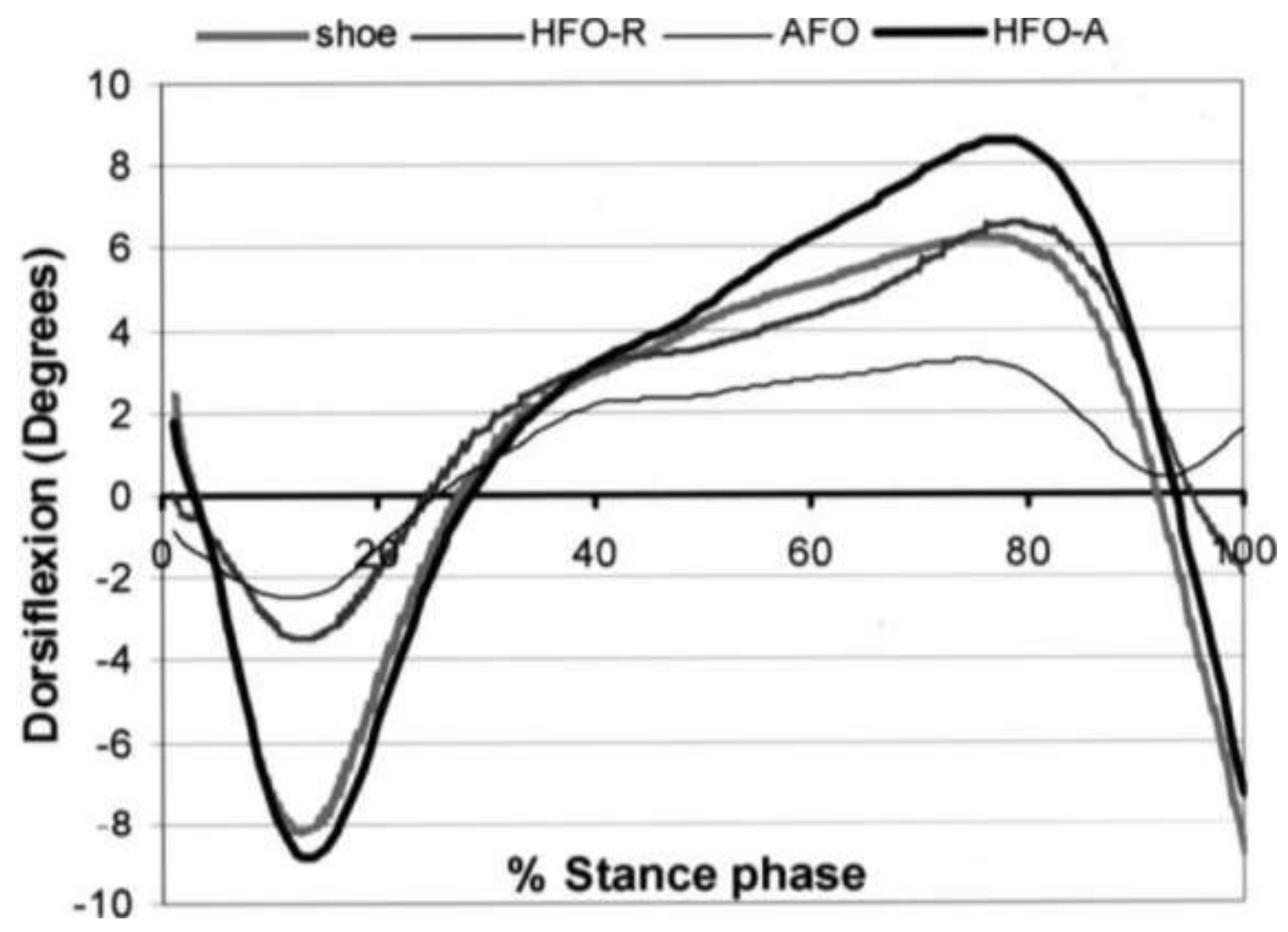

Figure 2-9. Ankle Motion in the Sagittal Plane with Various Orthoses

The Solid AFO and Rigid Hindfoot Orthosis (HFO-R) reduced sagittal plane motion compared to the Articulating Hindfoot Orthosis (HFO-A) and unbraced shod conditions. Reprinted with permission. Kitaoka, H. B., Crevoisier, X. M., Harbst, K., Hansen, D., Kotajarvi, B., \& Kaufman, K. (2006). The effect of custom-made braces for the ankle and hindfoot on ankle and foot kinematics and ground reaction forces. Arch Phys Med Rehabil, 87(1), 130-135. https://doi.org/10.1016/j.apmr.2005.08.120 [17] 


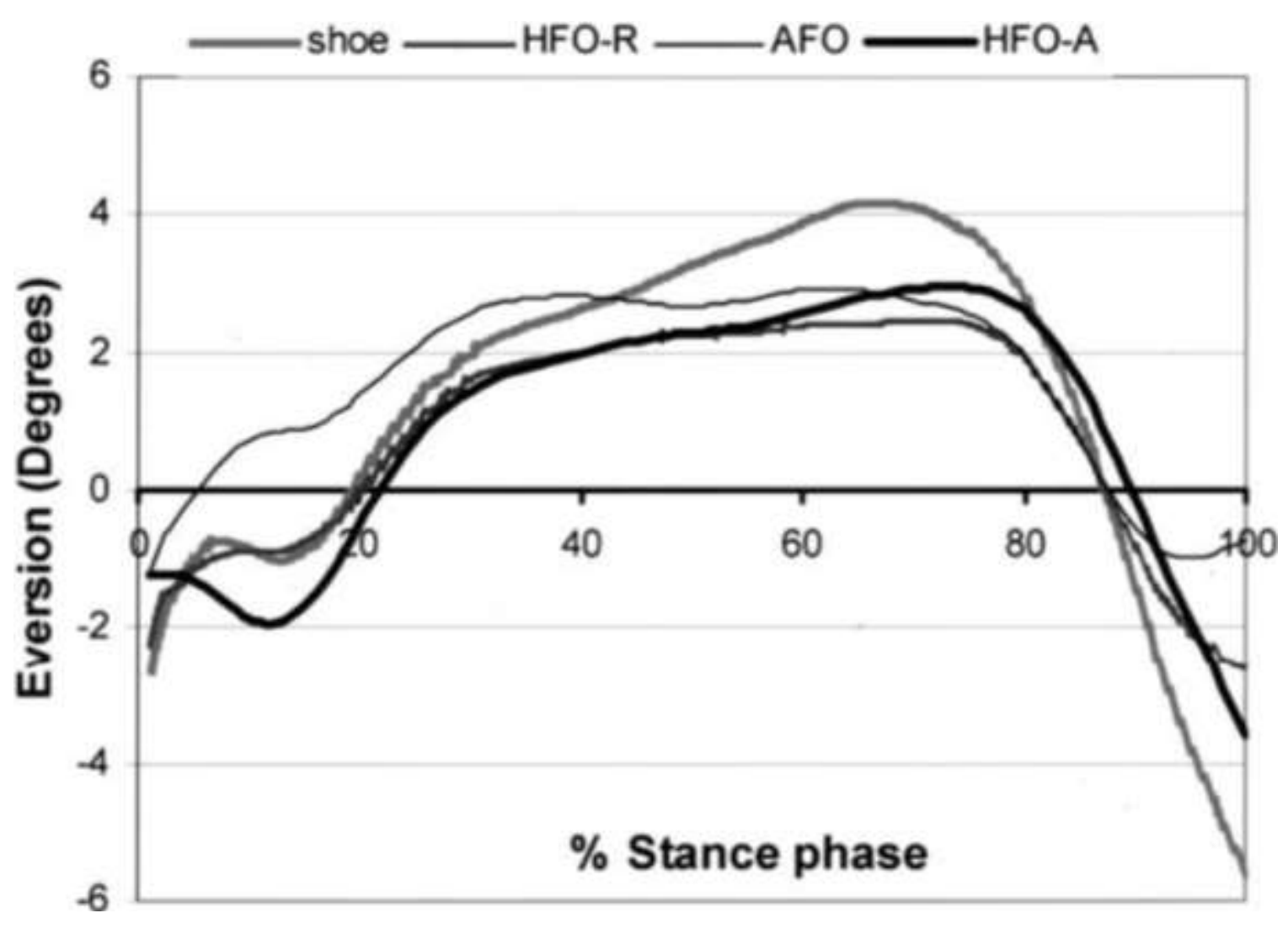

Figure 2-10. Ankle Motion in the Frontal Plane with Various Orthoses

All 3 orthoses reduced the maximal ankle inversion and total frontal plane motion compared with the unbraced shod condition. Reprinted with permission. Kitaoka, H. B., Crevoisier, X. M., Harbst, K., Hansen, D., Kotajarvi, B., \& Kaufman, K. (2006). The effect of custom-made braces for the ankle and hindfoot on ankle and foot kinematics and ground reaction forces. Arch Phys Med Rehabil, 87(1), 130-135.

https://doi.org/10.1016/j.apmr.2005.08.120 [17] 
two decades.[67] During the procedure, surgeons remove any bone spurs and install an external frame to physically distract the damaged joint surfaces about $5 \mathrm{~mm}$ apart, which is then worn for about 3 months. This immobilization distraction procedure is typically performed for the younger, more active individuals as a means for delaying the need for arthrodesis or arthroplasty.[68]

Experts agree that distraction and motion of damaged human ankles can promote joint remodeling, decrease pain, and improve joint function.[63-65,69] For the foot and ankle, however, there are almost no conservative "offloading" devices available on the market. The only known weight-bearing device is the Patellar Tendon Bearing cast or orthosis (PTB), which is reported to reduce pain and discomfort associated with belowknee fractures and degenerative foot and ankle conditions like osteoarthritis.[70,71] The PTB can significantly offload axial loads passing through the lower leg using a combination of design features. Specifically, the PTB is designed to engage with anatomical landmarks (i.e., weight-bearing through the patellar tendon) while providing under-foot gapping (heel clearance) and high levels of cushioning (Figure 2-11). The amount of offloading provided by the PTB can vary greatly with design alterations (Figure 2-12).[71-75] Like other AFOs, the PTB limits ankle motion in all planes either partially or completely. In recent years, this device has fallen out of common practice due to improvements in bracing technology and more affordable alternative treatment options.

\section{Dynamic Ankle Orthosis}

A conservative treatment option that preserves joint mobility while offloading the lower limb to alleviate pain is not currently available on the market. The DAO (Figure 2-13) was designed to address this unmet need and provide the coupled benefits of foot and ankle offloading while supporting the functional range of motion of the natural ankle joint.[76] The DAO was constructed with a custom-molded calf sleeve and a modified shoe, which grip the anatomy of the lower limb above and below the ankle. These sections were connected by pneumatic (air) cylinders, which apply a controlled distractive force across the ankle joint when pressurized, and anatomically positioned ball joints, which allow a functional range of ankle motion. When activated, the DAO offloads longitudinal forces passing through the foot and ankle by up to $30.5 \%$ in quiet stance without changing the resistance to moving the ankle through a functional range of motion (see Chapter 3).[77] During level-walking, the DAO reduces loads under the braced foot by up to $24 \%$ while allowing more natural frontal and sagittal plane ankle motion in healthy young adults than the standard of care AFO (see Chapter 4).[78] Finally, the DAO improves symptoms of pain and function in cases of osteoarthritis, posterior tibialis tendon dysfunction, postoperative pain, ankle sprains, and nerve disorders (see Chapter 5). 


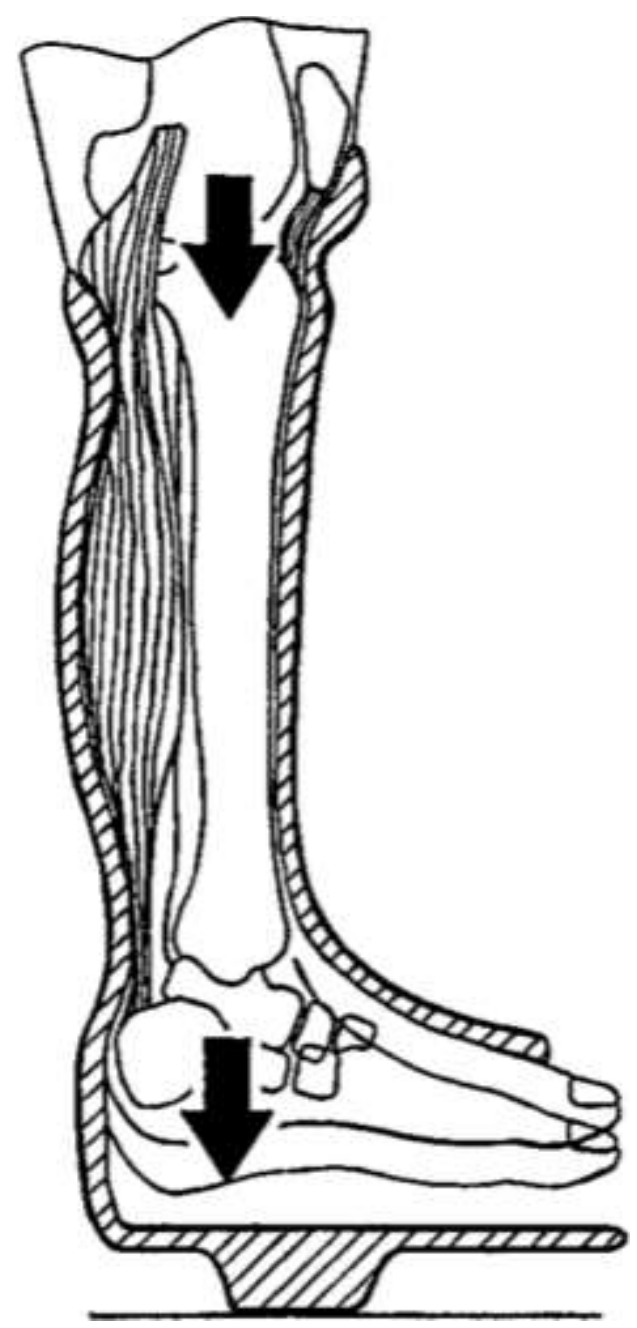

Figure 2-11. Diagram of the Offloading Effect in the PTB Cast and AFO Sliding of the leg occurs within the PTB upon loading (arrow) until suspension occurs. Reprinted with permission. Tanaka, H., Nagata, K., Goto, T., Hoshiko, H., \& Inoue, A. (2000). The effect of the patellar tendon-bearing cast on loading. J Bone Joint Surg Br, 82(2), 228-232. https://doi.org/10.1302/0301-620x.82b2 .9443 [75] 


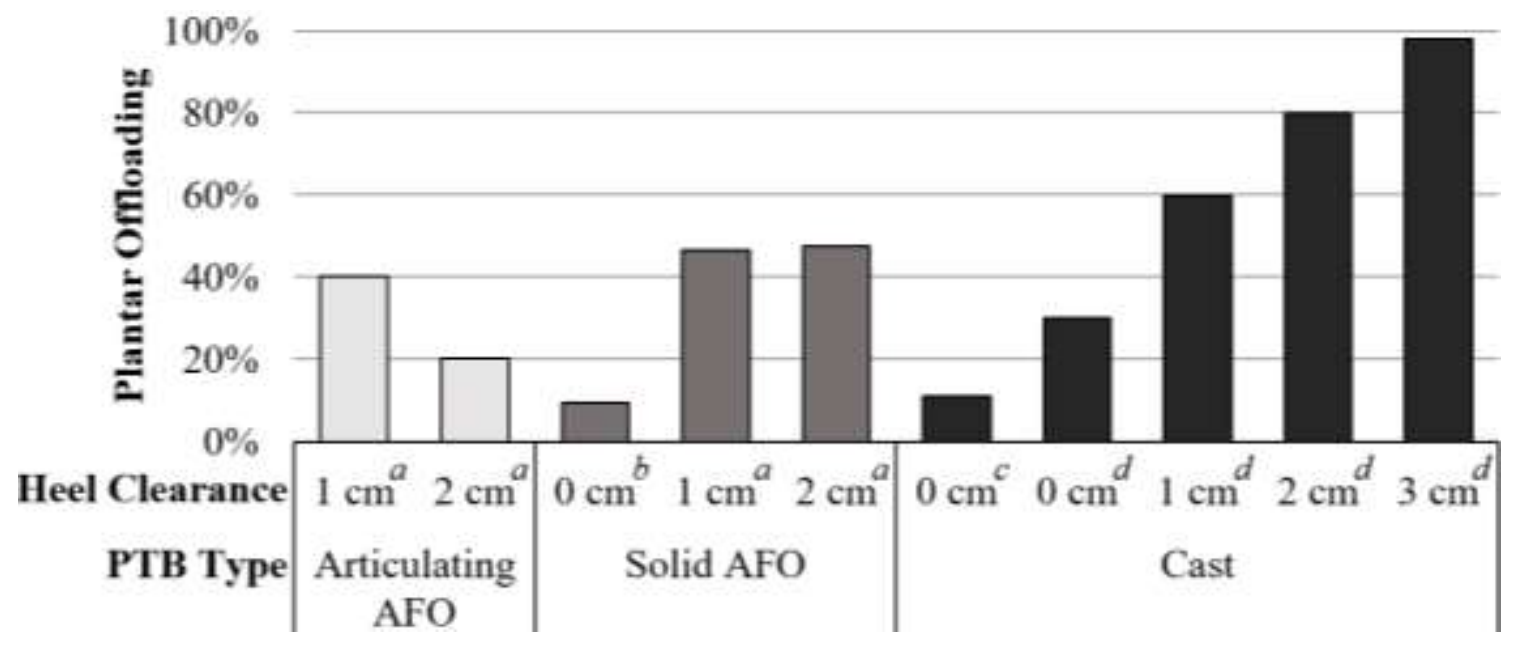

Figure 2-12. Combined Effect of PTB Design and Heel Clearance on Plantar Offloading

Source Data: "a” Alimerzaloo, F., Kashani, R. V., Saeedi, H., Farzi, M., \& Fallahian, N. (2014). Patellar tendon bearing brace: combined effect of heel clearance and ankle status on foot plantar pressure. Prosthet Orthot Int, 38(1), 34-38.

https://doi.org/10.1177/0309364613486916 [71] "b” Wanamaker, A. B. (2013).

Biomechanical comparison of lower limb unloading between common modalities of ankle foot orthoses. Retrieved from https://digitalcommons.du.edu/etd/685/ on December 5, 2016. [73] "c" Aita, D., Bhave, A., Herzenberg, J. E., Paley, D., \& Cannada, L. (1998). The load applied to the foot in a patellar ligament-bearing cast. J Bone Joint Surg Am, 80(11), 1597-1602. https://doi.org/10.2106/00004623-199811000-00005 [74] "d" Tanaka, H., Nagata, K., Goto, T., Hoshiko, H., \& Inoue, A. (2000). The effect of the patellar tendon-bearing cast on loading. J Bone Joint Surg Br, 82(2), 228-232.

https://doi.org/10.1302/0301-620x.82b2 .9443 [75] 

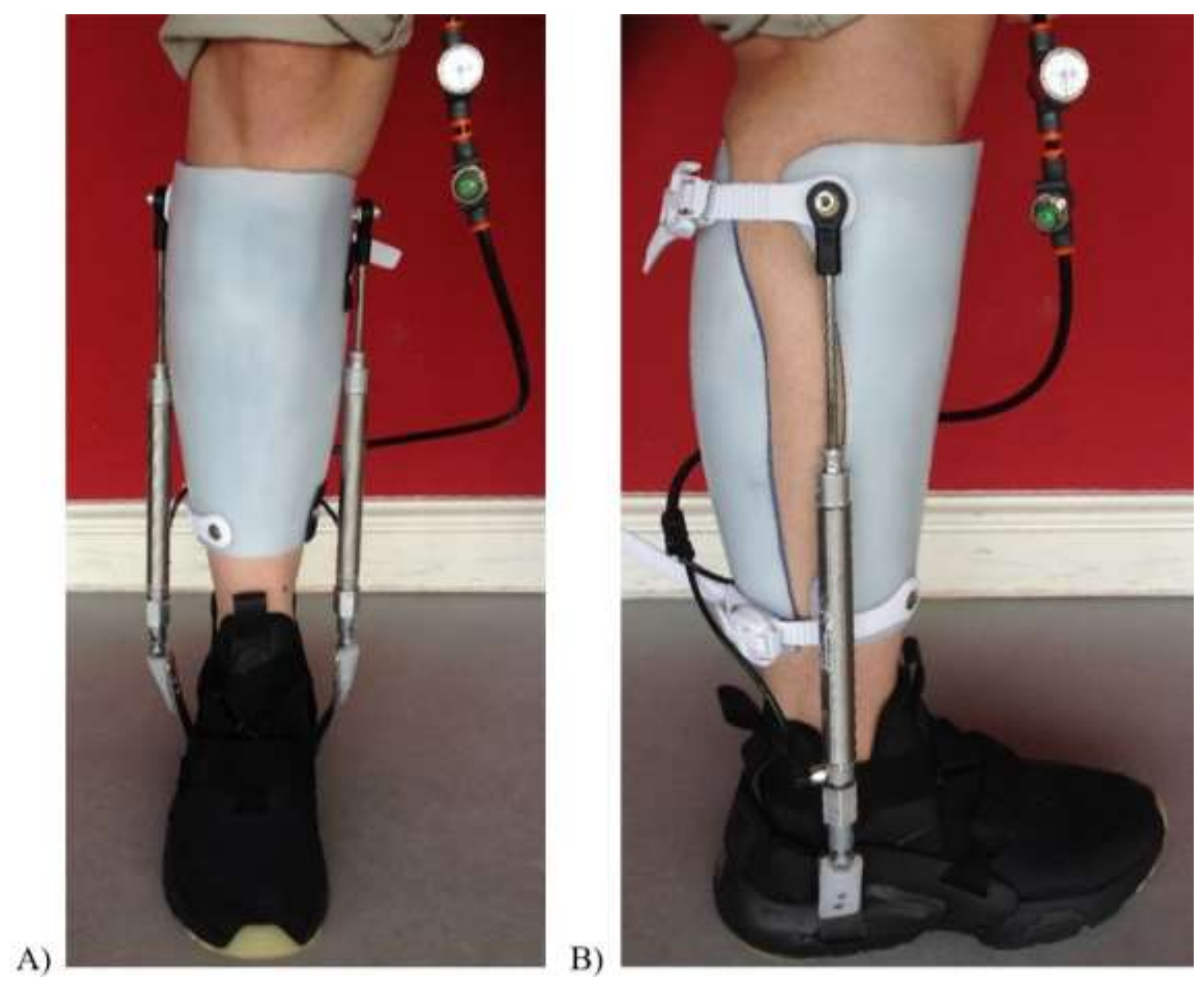

Figure 2-13. Dynamic Ankle Orthosis

A) Front View and B) Side View. 


\section{CHAPTER 3. DESIGN AND VALIDATION TESTING OF A DYNAMIC ANKLE ORTHOSIS ${ }^{1}$}

This chapter presents the design, development, and benchtop validation testing of the Dynamic Ankle Orthosis. This chapter was published in the Journal of Applied Bionics and Biomechanics.

\section{Introduction}

Ankle fractures represent a significant portion of orthopedic trauma injuries that occur in the United States. In 2014, over 60,000 work-related ankle injuries were reported to the U.S. Department of Labor with average costs up to $\$ 5,900$ per injury. $[33,34]$ Patients who sustain irreversible cartilage damage or joint instability from ankle injuries are likely to develop ankle arthritis. Affecting approximately $1 \%$ of adults, ankle osteoarthritis $(\mathrm{OA})$ is a degenerative disease that occurs when the cartilage layer in the joint wears away over time.[8] Unlike OA of other joints, $70 \%-80 \%$ of ankle OA cases are associated with a previous traumatic injury, a condition known as the Post-Traumatic Ankle Osteoarthritis (PTOA).[8,9] Long-term physical impairments, joint pain, and reduced quality of life are all possible outcomes of this debilitating disease. Ankle OA creates progressive pain within the ankle joint, inflammation of the joint, and substantial decrease in overall range of motion and stability of the joint due to joint articular cartilage erosion. Invasive treatment options include ankle replacement (arthroplasty) and ankle fusion (arthrodesis). There are approximately 80,000 ankle fusions and 16,000 ankle replacements performed annually, and the estimated economic burden of foot and ankle surgery in the Medicare population was \$11 billion in 2011.[42,43] However, surgical procedures are joint sacrificing and typically done as a last resort. The pain and inflammation associated with PTOA can be managed noninvasively using modified footwear and orthotics such as an Ankle-Foot Orthosis (AFO) (i.e., brace).

Traditional AFOs can have solid joints with locked ankle position to prevent uncontrolled plantarflexion (i.e., foot drop due to weak dorsiflexors) or toe drag during walking. These solid AFOs are often comprised of lightweight plastic material that has been custom molded to fit the patient and maintain a desired ankle position. AFOs can also be designed with articulating pin joints to provide either free or controlled sagittal plane ankle motion. By incorporating a feature that provides a physical plantarflexion stop, plantarflexion resistance, or dorsiflexion assistance, the brace can prevent excessive plantarflexion while permitting natural progression of the tibia over the foot and natural dorsiflexion of the foot during the stance phase of walking. Articulating AFOs can be made with plastic materials or using metal and leather components adapted to the user's existing footwear. Ultimately, the biomechanical function and design of the prescribed

\footnotetext{
${ }^{1}$ Reprinted from final submission with open access permission. Chung, C. L. \& DiAngelo, D. J. (2018). Design and validation testing of a dynamic ankle orthosis. MOJ App Bio Biomech, 2(3), 210-215. https://doi.org/10.15406/mojabb.2018.02.00069 [77].
} 
AFO must match the needs of the patient.[45] A standard double upright AFO (DUAFO) is commonly prescribed to treat PTOA symptoms. These braces provide stabilization of the ankle by limiting ankle walking motion (i.e., sagittal plane).[17] However a standard DUAFO (Figure 3-1) has important limitations in that it functions primarily to stabilize the ankle joint with restricted motion and provides minimal to no off-loading. Reduced axial loads through the ankle have been shown to be effective in reducing the pain and discomfort of ankle arthritis.[70] A device that preserves ankle mobility while offloading the ankle joint for alleviating the pain of PTOA is not currently available on the market.

A new Dynamic Ankle Orthosis (DAO) was designed to provide the coupled benefits of ankle offloading while supporting the functional range of motion (ROM) of the natural ankle joint to noninvasively alleviate pain due to ankle osteoarthritis without increasing the muscle effort to move the ankle. The objectives of this study were to confirm the brace offloading effect during static double stance and quantify the muscle effort needed to move the ankle through a functional range of motion in the sagittal plane (i.e., dorsiflexion and plantarflexion).

\section{Methods}

The design goals of the DAO device were two-fold: 1) offload the ankle joint by applying a distractive force between the foot and proximal tibia and 2) allow for a functional range of motion at the natural ankle joint without increasing the muscle effort required to move the ankle. The DAO (Figure 3-2) consisted of three main components: a calf sleeve, a foot plate, and two pneumatic cylinders that attached to the calf sleeve and foot plate via ball joints and tie rod ends and served as a distractive force mechanism (DFM) (See Appendix A for specifications; Table A-1 for components, Table A-2 for equipment). The DAO applied a distractive force along the axis of the lower leg and through the rotational axis of the device to create an offloading effect. The two cylinders were connected with tubing so that air could move between cylinders as needed (i.e., when one cylinder rod collapsed, the other cylinder rod extended). This action allowed the actuator force to remain constant during weight-bearing as the foot moved into inversion and eversion. The ball joints and tie rod ends, coupled with the way the actuators were configured, allowed for functional ranges of inversion, eversion, dorsiflexion, and plantarflexion of the ankle joint. Based on recommendations of local Orthotists (Center of Prosthetics and Orthotics Inc.) the brace was designed to offload at least $10 \%$ of the user's bodyweight (BW). Finally, the arrangement of the device's rotational axis with the anatomical axis of the ankle joint allowed for a functional walking range of dorsiflexion $\left(\sim 10^{\circ}\right)$ and plantarflexion $\left(\sim 20^{\circ}\right)$ (i.e., rotating toes up and down) (Figure 3-3), and some amount of inversion and eversion (i.e., side-to-side rotation) (Figure 3-4). To test the effectiveness of the DAO to meet the design goals, two experiments were performed: a first experiment was conducted to confirm how much offloading the DAO provided to the lower limb, and a second experiment was conducted to test the changes in resistance to ankle plantarflexion and dorsiflexion compared to the native ankle condition. The methodology of these experiments is provided in the following sections. 


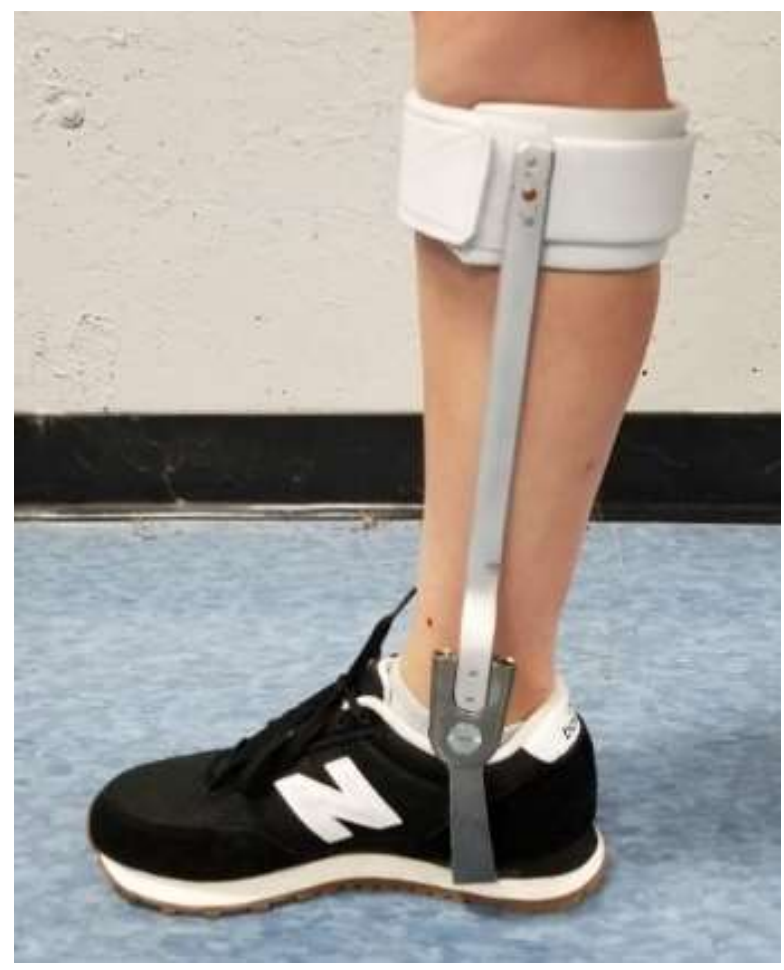

Figure 3-1. Standard of Care Leather and Metal Double Upright Ankle-Foot Orthosis

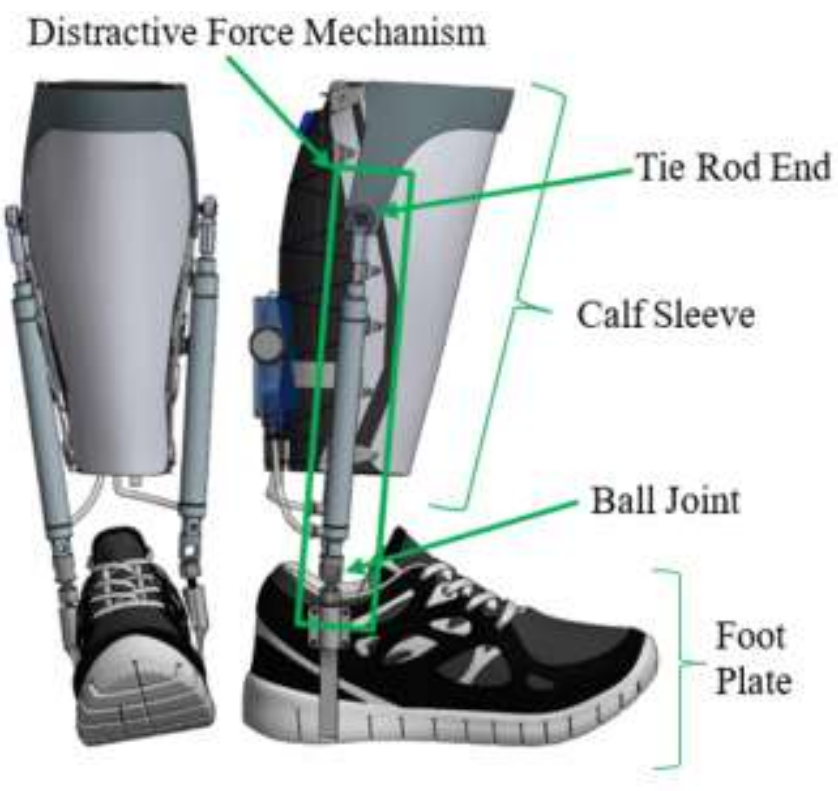

B)

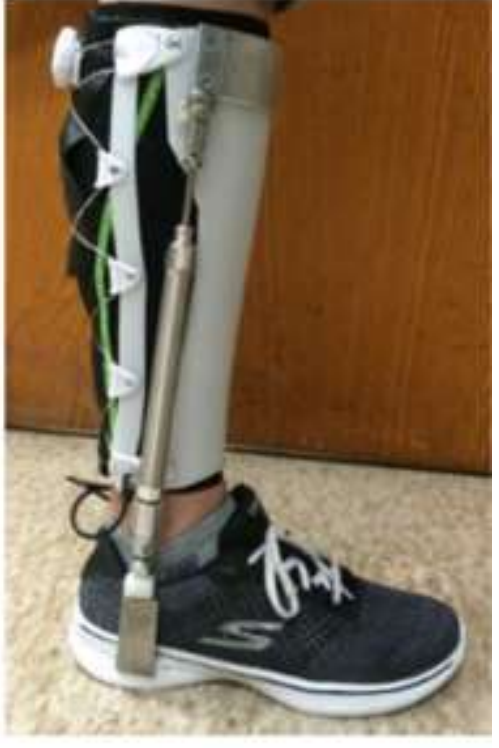

Figure 3-2. Dynamic Ankle Orthosis

A) Concept Model and B) Prototype. 

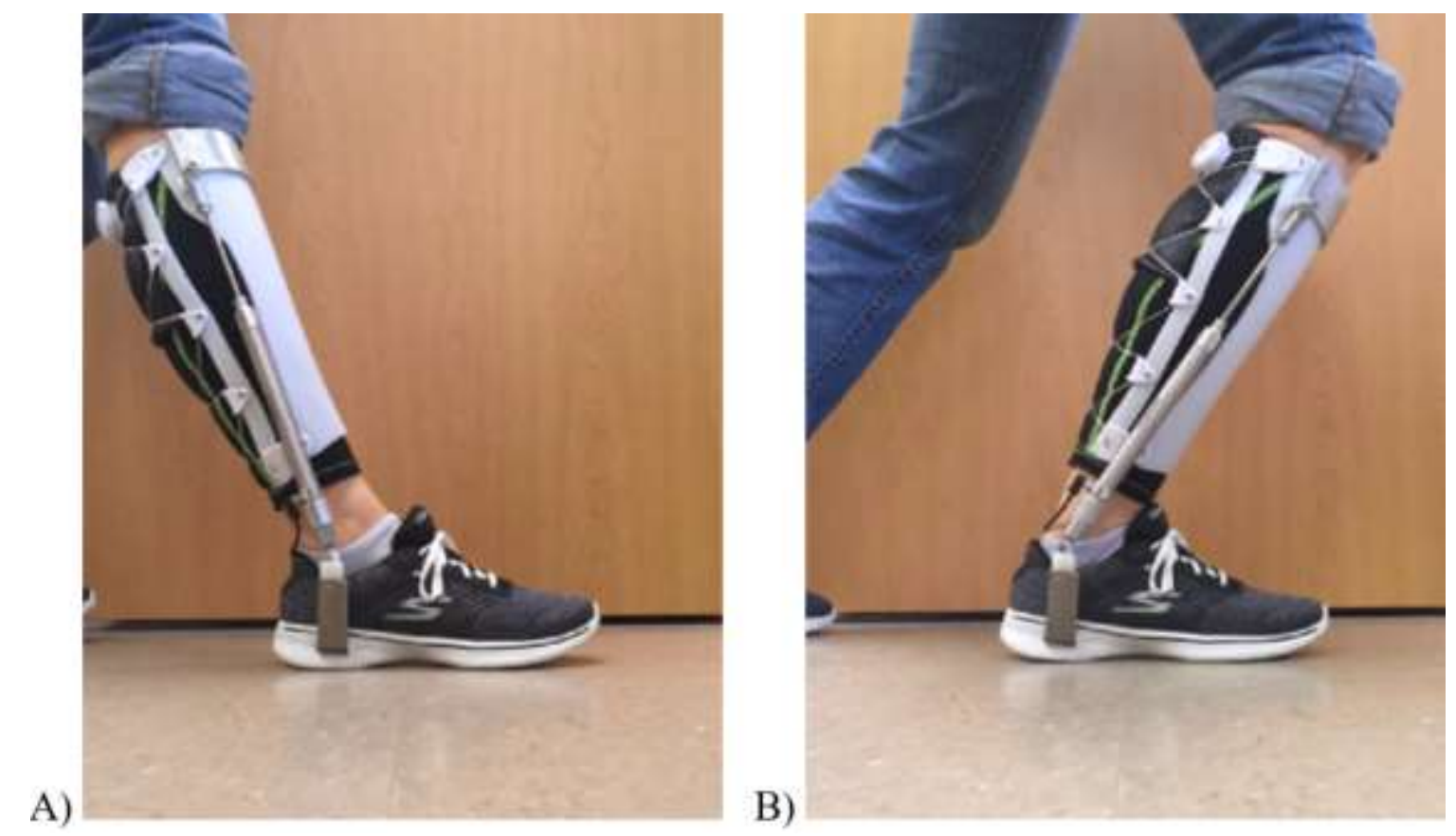

Figure 3-3. Sagittal Plane Range of Motion of the DAO During Weight-Bearing A) Maximum Plantarflexion and B) Maximum Dorsiflexion.
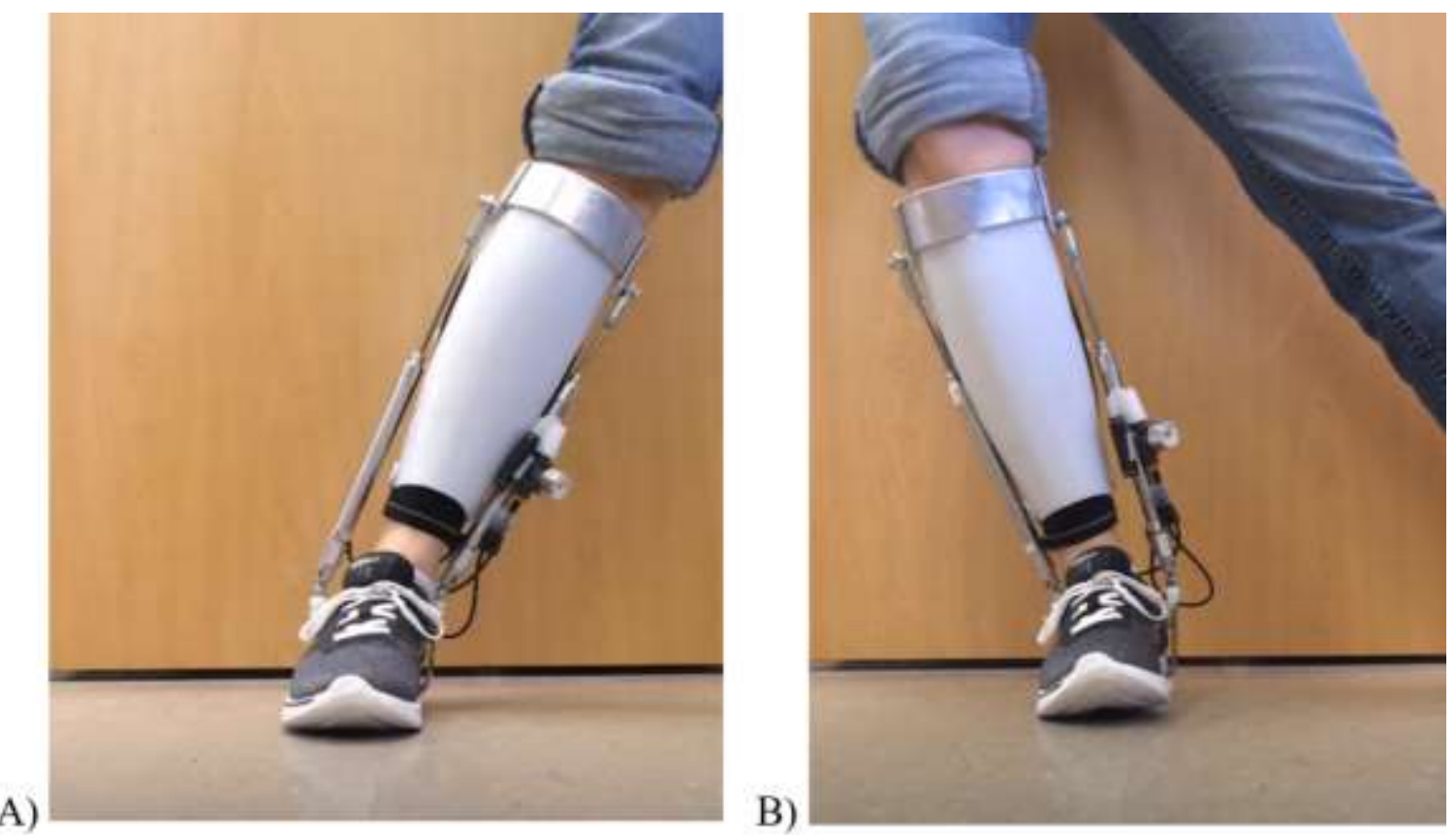

Figure 3-4. Frontal Plane Range of Motion of the DAO During Weight-Bearing A) Maximum Inversion and B) Maximum Eversion. 


\section{Offloading Validation}

This experiment was performed in two parts: 1) define cylinder pressure-output force relationship of the two cylinders alone, and 2) define the cylinder pressureoffloading relationship of the brace. The pressure-output force relationship of the standalone pneumatic cylinders was first characterized (Figure 3-5). A plate was created with two clearance holes to place the 7/16-20 threaded ends of the cylinders through. A corresponding nut was then used to secure pneumatic cylinders to the plate. They were each attached to a vertical fixture for positioning, which was securely attached to the platform of a robotic test frame and placed beneath a load cell (Model 100M40 six-axis load cell; JR3 Inc., Woodland, CA). The cylinders were connected in series in the same manner as when attached to the DAO. By connecting pneumatic cylinders in series, equivalent pressures were delivered to the two cylinders, corresponding to the pressure value shown on the pressure gauge (i.e., when the pressure gauge showed 40 PSI, both cylinders were inflated to a pressure of 40 PSI). Both cylinders acted on the load cell, so the reported values show the total amount of force delivered by both cylinders. The compressive force on the load cell was acquired at various cylinder pressure values: 40, 50, 60, 70, and 80 PSI. Each targeted pressure value was tested four times, and the load cell readings were exported and processed in Microsoft Excel (2013). The pneumatic cylinders were emptied of their pressures between all runs and inflated to the targeted pressure to begin each new test.

To quantify the biomechanical offloading capabilities of the DAO as a function of pneumatic pressure relative to bodyweight, a testing fixture was created to measure the amount of load relief that the DAO provided (Figure 3-6). Two vertical fixture plates were mounted to a base plate and positioned on either side of the braced leg. A small mounting plate was attached to the vertical fixture plates, and the lower ends of the DFM pneumatic cylinders were attached to the mounting plate via tie rod ball joint connectors. The mounting plates were adjusted so that the height and location of the connectors were comparable to that of the two ankle malleoli. Two load cells (Model 100M40 and Model 67M25S3; JR3 Inc., Woodland, CA) were bolted to the top of the base plate, and wooden planks were then attached to the top of each of the load cells to give the user somewhere to stand. The user stood with their feet approximately shoulder width apart with one foot on one load cell and the other foot on the other load cell. With the lower end of the cylinders attached to the ball joint connectors and side mounting plates, the subject donned the calf sleeve of the DAO around their right leg. In this configuration, any brace force introduced by pneumatic cylinders bypassed the load cell under the braced limb and transferred to the base plate.

Testing was performed in static double stance with each foot positioned on top of a single load cell. A custom program recorded load cell readings over a specified period. At the start of each test, approximately five seconds of load data were collected without brace activation. The pneumatic cylinders were activated to a specific pressure to create a brace force, and testing was continued for twenty seconds. The pneumatic cylinders were inflated to five different pressures: 40, 50, 60, 70, and 80 PSI and tested ten times at each

level. Brace force $\left(\mathrm{F}_{\mathrm{b}}\right)$ was calculated for each run by taking the user's bodyweight, or the 


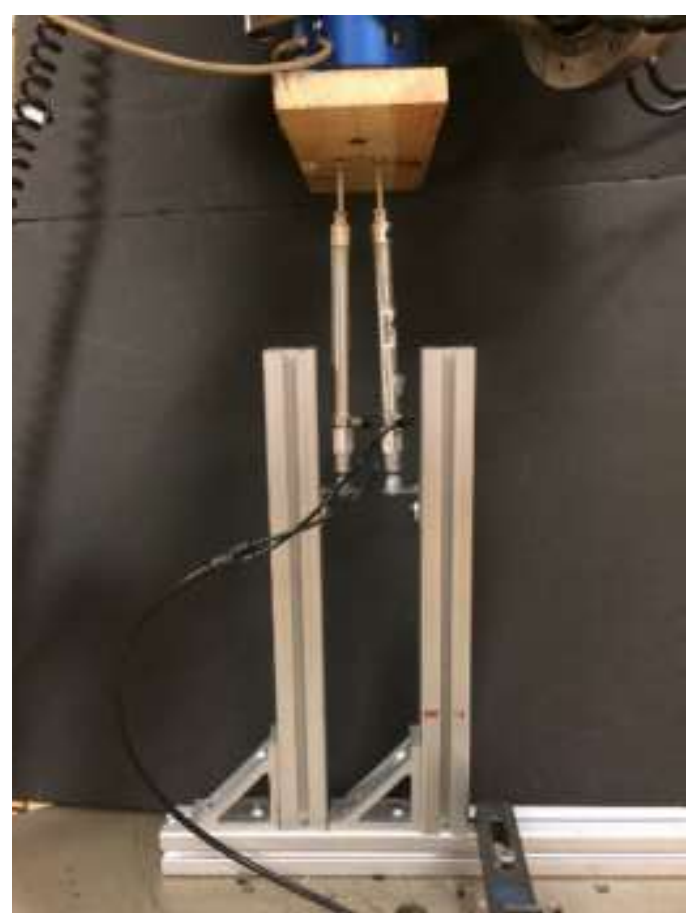

Figure 3-5. Setup for Characterizing Cylinder Pressure-Output Force Relationship
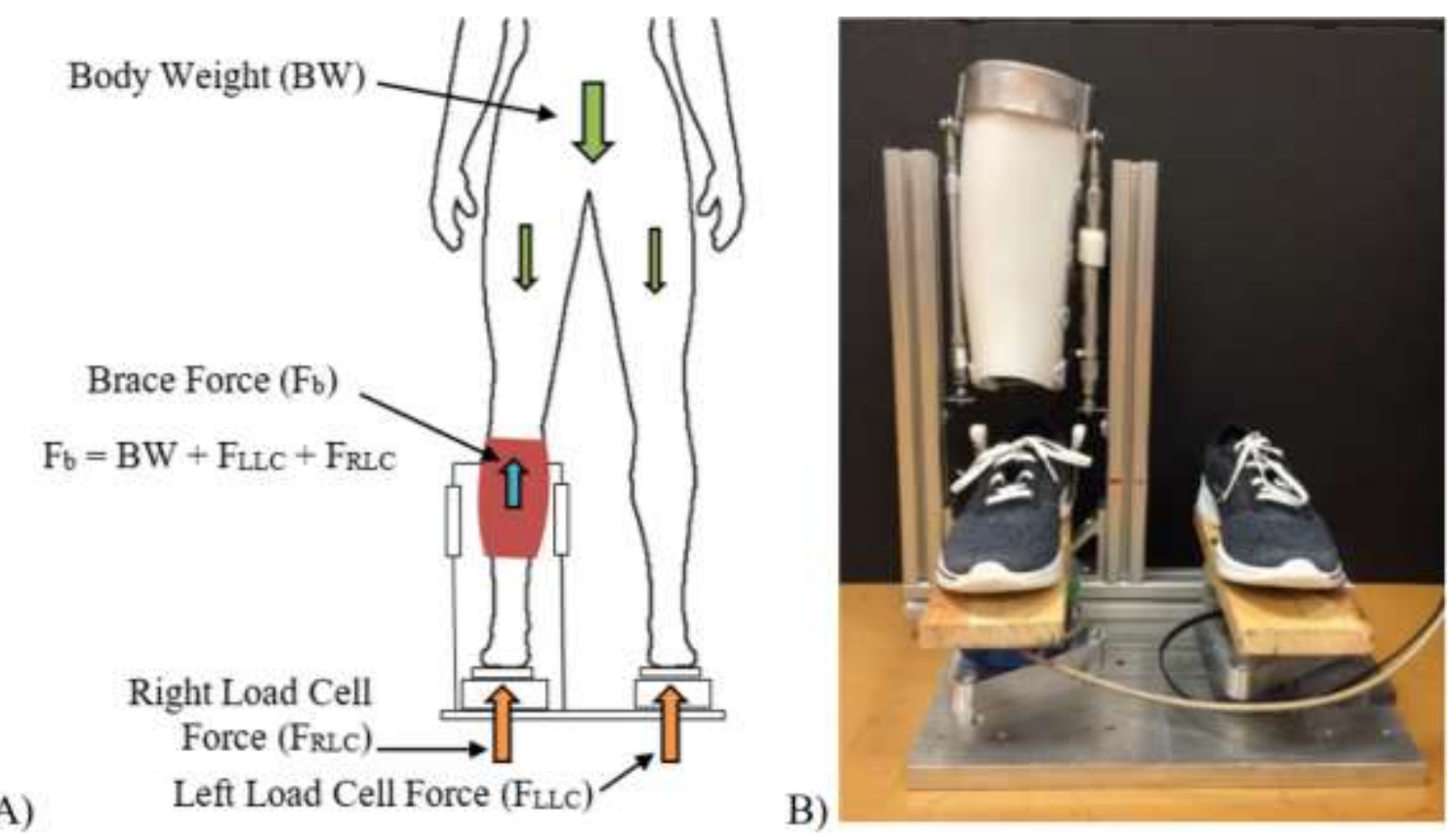

Figure 3-6. Setup for Offloading Validation A) Free Body Diagram and B) Test Fixture. 
sum of the readings of the left load cell force $\left(\mathrm{F}_{\mathrm{LLC}}\right)$ and of the right load cell force $\left(\mathrm{F}_{\mathrm{RLC}}\right)$ before brace activation and subtracting out the sum of the two load cell readings after brace activation. Brace Efficiency, or how well DAO transferred offloading forces to the limb, was calculated using the brace force $\left(\mathrm{F}_{\mathrm{b}}\right)$ of the DAO and total cylinder force provided by pneumatic cylinders in the first part of this experiment.

\section{Mobility Validation}

Validation of the second design goal of the DAO, i.e., to provide a functional range of ankle motion without increasing the muscle effort required to move the ankle joint, was done by analyzing the effect of the DAO on the ankle's resistance to passive sagittal plane motion. A Biodex Dynamometer (System 4 Pro Model: 850-000, Biodex Medical Systems, NY, USA) was set to passively drive the ankle in plantarflexion and dorsiflexion with and without the DAO worn. The user sat in a chair, and a limb support pad was placed under the thigh so that the lower leg approached the machine parallel to the floor (Figure 3-7). The right foot was placed on a foot plate and securely attached to limit motion in the foot. The foot plate was then adjusted so that the dynamometer of the Biodex unit was aligned with the lateral malleolus of the right ankle. This was done so that the Biodex unit rotated about the rotational axis (RA) of the user's ankle. The Biodex unit was set to rotate the ankle between two set points at a set speed. The two points were set before each run by the user.

The foot was initially positioned at a neutral angle, perpendicular to the long axis of the leg, and then the user sets maximum plantarflexion and dorsiflexion angles. The machine rotated back and forth between these maximum angles for a set number of cycles and measured the moments experienced by the machine while moving the foot to these points. The data sets attained from the tests were the angular positions and the corresponding moment values recorded by the dynamometer. All tests were run at an angular velocity of 30 degrees/sec. For all tests, the ankle was rotated to at least 10 degrees of dorsiflexion and 20 degrees of plantarflexion. The polarity of the measured moment values depends on the direction of the resistive moment. Dorsiflexor moments (directed towards the top of the foot) were measured as negative resistive moments, and plantarflexor moments (directed towards the bottom of the foot) were measured as positive resistive moments). So as the foot passively moved into dorsiflexion, a resistive plantarflexor moment was generated by the soft tissue of the calf and ankle.

The foot was placed in the Biodex machine without the DAO donned. The straps were securely tightened at the foot to ensure that rotation occurred at the ankle joint. Three tests were run, each for thirty seconds with the system angular velocity set to 30 degrees/sec. The mean moments and standard deviations were then calculated at 5 degree increments from - 10 degrees to 20 degrees. Moment values for the plate by itself were subtracted out so the reported moment values accurately represent what was added to the Biodex system. These values provided a baseline to compare the results of the different bracing conditions to that of the foot alone. Next, six different bracing conditions were tested. The user sat in the chair as described above, with the DAO donned and the foot 


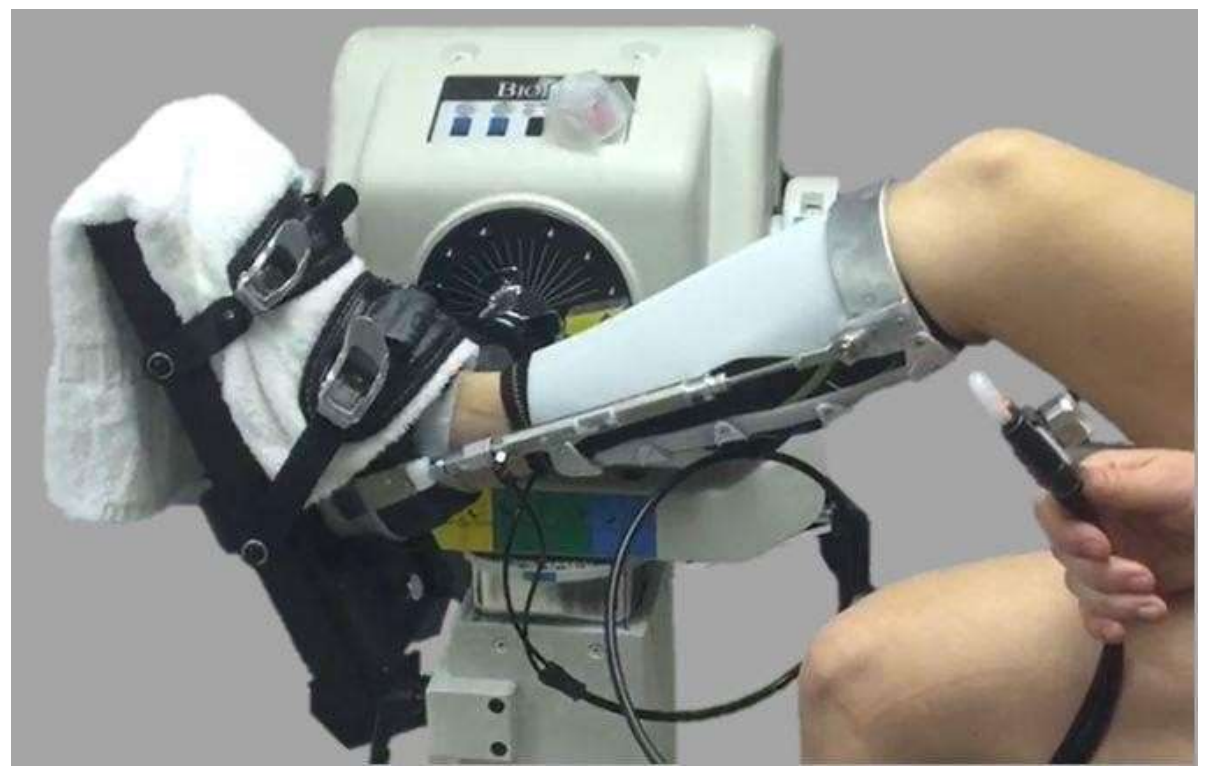

Figure 3-7. Setup for Mobility Validation of DAO Using an Isokinetic Dynamometer 
secured to the foot plate in the neutral position. Each test was run for 30 seconds and contained between 7 and 10 full revolutions of the foot, moving from maximum dorsiflexion to maximum plantarflexion and back. Tests were run with the pneumatic cylinders filled to $0,40,50,60,70$, and 80 PSI values. Each pressure setting was run three times and the recorded output values were the moments and the corresponding angular position values.

Moment values at $-10,-5,0,5,10$, and 15 degrees were used for analysis. The mean resistive ankle moments were compared amongst all bracing conditions using a Shapiro-Wilk Normality Test, Equal Variance Test, One-Way ANOVA, and Holm-Sidak Post-Hoc Analysis (Sigma Plot Version 13.0; Systat Software Inc., CA). Significance was set at an alpha level of 0.05 . The null and alternative hypotheses were defined as follows: $\mathrm{H}_{\mathrm{o}}$ : There was no difference in mean resistive ankle moment between bracing conditions; $\mathrm{H}_{\mathrm{A}}$ : There was a difference in mean resistive ankle moment between bracing conditions.

\section{Results}

\section{Offloading Results}

The mean output force of the pneumatic cylinders at varying levels of cylinder pressure are shown in Table 3-1 along with the mean transferred brace force, $\mathrm{F}_{\mathrm{b}}$, that was computed from the load cell data per the free body diagram of Figure 3-6. Table 3-1 also shows the brace efficacy expressed as a percentage of the cylinder force that the brace was able to transfer in the form of a bracing force, $\mathrm{F}_{\mathrm{b}}$. The percent bodyweight offloaded by the brace force $\mathrm{F}_{\mathrm{b}}$, using the mean bodyweight of $490 \mathrm{~N}$ is shown in Figure 3-8.

\section{Mobility Results}

Following in Table 3-2 are the mean moment values for the native ankle and braced conditions. At each five degree increment in both directions, the mean moment was compared amongst all different bracing conditions. An angular ankle position of 0 degrees corresponds to the neutral ankle position where the foot makes a 90-degree angle with the long axis of the lower leg. Negative angle values indicate dorsiflexion and positive values plantarflexion. A box plot representation of the resistive ankle moments measured at each angular ankle position for each of the bracing conditions is shown in

Figure 3-9. The mean resistive ankle moment for all DAO bracing conditions were significantly different from "Native Ankle" except for "DAO 0 PSI" condition. 
Table 3-1. Activated Cylinder Pressures with Corresponding Output Force for Two Pneumatic Cylinders, Computed Transferred Brace Force, and Brace Efficacy

\begin{tabular}{cccc}
\hline Pressure [PSI] & Cylinders Output Force [N] & $\mathbf{F}_{\mathbf{b}}[\mathbf{N}]$ & Brace Efficiency \\
\hline 40 & $65.5 \pm 2.5$ & $54.7 \pm 7.3$ & $83.4 \%$ \\
50 & $87.2 \pm 0.7$ & $75.1 \pm 3.7$ & $86.1 \%$ \\
60 & $111.9 \pm 2.2$ & $106.9 \pm 5.9$ & $95.4 \%$ \\
70 & $135.5 \pm 2.6$ & $123.5 \pm 5.9$ & $91.4 \%$ \\
80 & $155.3 \pm 1.3$ & $148.0 \pm 7.9$ & $95.5 \%$ \\
\hline
\end{tabular}

Notes: Mean $\pm \mathrm{SD} ; \mathrm{F}_{\mathrm{b}}$ is brace force computed from known bodyweight and load cell readings.

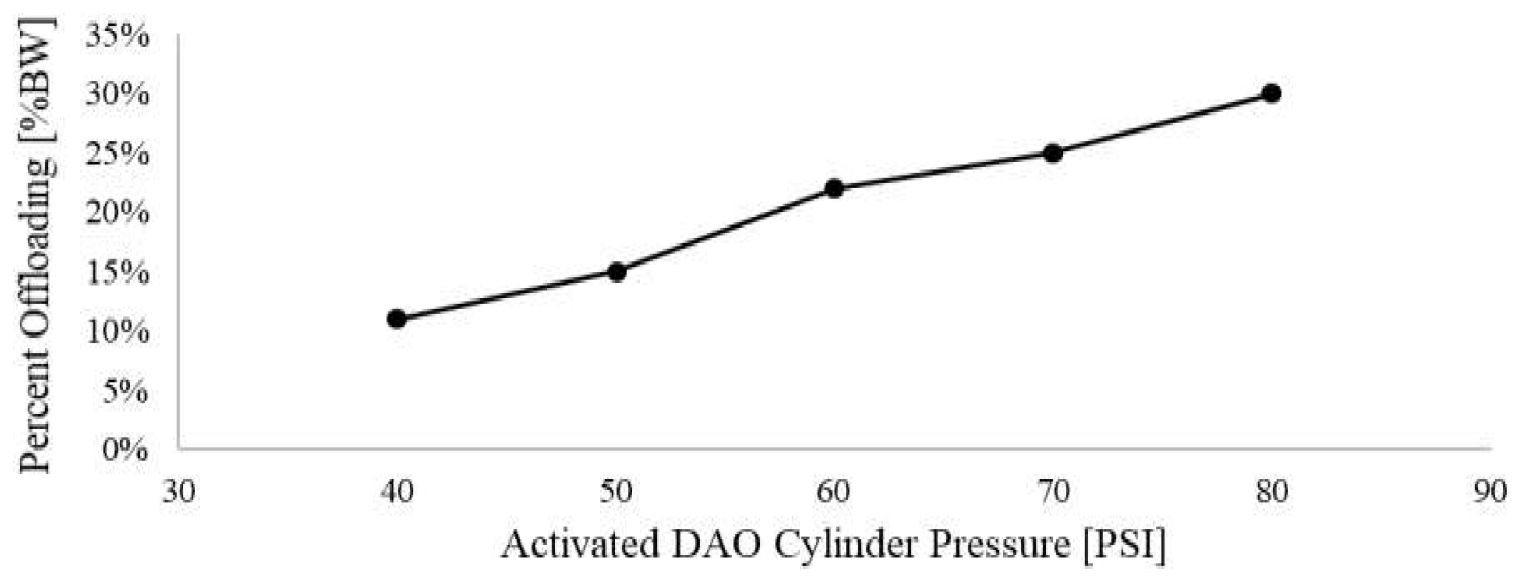

Figure 3-8. Percent of Bodyweight Offloaded by the DAO at Varying Levels of Cylinder Pressure 
Table 3-2. Native Ankle and Braced Ankle Moment Values at $5^{\circ}$ Increments of Angular Ankle Position

\begin{tabular}{|c|c|c|c|c|c|c|c|}
\hline \multirow[b]{2}{*}{ Condition } & \multicolumn{2}{|c|}{ Dorsiflexion (-) } & \multirow{2}{*}{$\begin{array}{c}\text { Neutral } \\
0^{\circ}\end{array}$} & \multicolumn{4}{|c|}{ Plantarflexion (+) } \\
\hline & $-10^{\circ}$ & $-5^{\circ}$ & & $5^{\circ}$ & $10^{\circ}$ & $15^{\circ}$ & $20^{\circ}$ \\
\hline Native Ankle & 6.0 & 4.5 & 3.7 & 3.1 & 2.4 & 1.9 & 1.5 \\
\hline DAO 0 PSI & 4.2 & 3.3 & 2.6 & 1.8 & 1.2 & 0.7 & 0.1 \\
\hline DAO 40 PSI & 2.6 & 1.8 & 0.9 & 0.4 & 0.0 & -0.5 & -1.1 \\
\hline DAO 50 PSI & 2.6 & 1.8 & 1.1 & 0.4 & 0.0 & -0.7 & -1.8 \\
\hline DAO 60 PSI & 2.6 & 1.6 & 0.8 & 0.3 & -0.3 & -0.9 & -1.9 \\
\hline DAO 70 PSI & 2.6 & 1.6 & 0.7 & 0.0 & -0.5 & -1.2 & -2.3 \\
\hline DAO 80 PSI & 1.8 & 0.8 & 0.0 & -0.7 & -1.4 & -2.2 & -4.2 \\
\hline
\end{tabular}

Note: Moment values expressed in Nm.

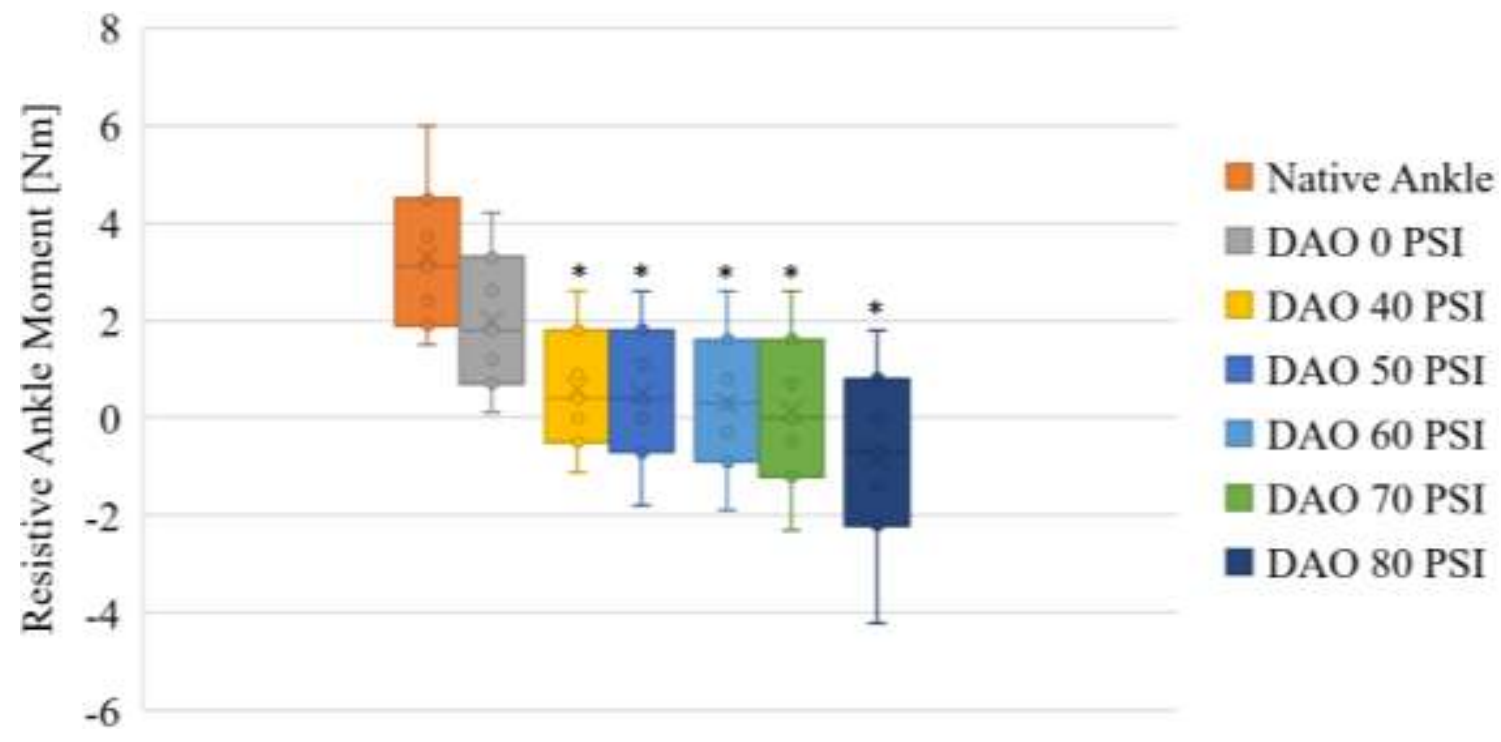

Figure 3-9. Box Plot of Resistive Ankle Moment Measured at $5^{\circ}$ Increments of Ankle Angular Position for Each Bracing Condition

*denotes a significant difference from Native Ankle mean resistive ankle moment $(\mathrm{p}<0.05)$ 


\section{Discussion}

\section{Offloading Capacity of the DAO}

The amount of force that the DAO relieved expressed as a percentage of bodyweight, ranged from $11.3 \%$ at 40 PSI to $30.5 \%$ at 80 PSI. It is currently unknown what amount of force relief at the ankle is clinically relevant, but through conversation with two orthotists, two general goals were set for the DAO. First, the DAO should provide axial unloading without compromising circulation or soft tissue integrity. The medical literature shows that during noninvasive ankle distraction no nerve damage was seen when tested up to $135 \mathrm{~N}$ for 1 hour. [79] This is not directly applicable as this experiment was performed on an unloaded ankle (i.e., non-weight-bearing) and the ankle would be under load with the DAO, but it still gave a reference of what was safe without causing nerve damage in an unloaded ankle. Second, a goal of at least $10 \%$ of bodyweight of force relief at the braced ankle was set as a mark of potential clinical efficacy. This test shows the ability of the DAO to provide offloading of the ankle. The DAO provided up to $148 \mathrm{~N}$ of brace force $F_{b}$ to the user which amounted to $30.5 \%$ of bodyweight. The DAO was also able to transfer cylinder force output to brace force $\mathrm{F}_{\mathrm{b}}$ at an $83-95 \%$ effectiveness rate. The DAO, therefore, accomplished both design goals set by the Orthotists.

\section{Mobility}

The DAO allowed for full functional ROM of ankle of 10 degrees of dorsiflexion and 20 degrees of plantarflexion.[22] The Biodex testing showed that there was a reduction in resistive torque at the ankle with increasing DAO cylinder pressure. The measured passive moment values for the baseline native ankle condition are supported by literature.[79] Furthermore, in agreement with our findings, Kay et al. observed that moderate-duration static stretching reduces the active and passive plantarflexor moment at the ankle.[79] Normality of the data was confirmed using a Shapiro-Wilk Normality Test $(p=0.469)$ and an Equal Variance Test $(p=0.953)$. A One-Way ANOVA showed that there is a difference between the mean resistive ankle moments of the bracing conditions $(p=0.001)$ with a power of 0.926. A Holm-Sidak Post- Hoc Analysis showed that the mean ankle moment for all DAO bracing conditions except "DAO 0 PSI" were significantly different from "Native Ankle." In other words, there was a reduction in baseline resistive moment at the ankle with increasing cylinder pressure, but no difference in the resistive moment of the ankle with the deactivated (unpressurized) brace.

Across the simulated range of motion (10 degrees dorsiflexion to 20 degrees plantarflexion), the native ankle experienced between 1.5 and $6.0 \mathrm{Nm}$ (4.5 $\mathrm{Nm}$ difference) of resistive moment. With the DAO donned and cylinders depressurized, between 4.2 and $0.1 \mathrm{Nm}$ (4.1 Nm moment difference) of resistive moment was measured during the motion. With the DAO donned and inflated to 50 PSI, between 2.6 and $-1.8 \mathrm{Nm}(4.4 \mathrm{Nm}$ 
moment difference) of resistive moment was measured during the motion. With the DAO donned and inflated to $80 \mathrm{PSI}$, between 1.8 and $-4.2 \mathrm{Nm}$ ( $6 \mathrm{Nm}$ moment difference) of resistive moment was measured during the motion. The increased moment at 20 degrees plantarflexion was likely due to the ball joints of DAO hitting their limit (i.e., the ball joints were constructed to permit a maximum of 20 degrees of motion). In all pressurized bracing conditions, the mean resistive moment was reduced compared to the native ankle. This finding suggests that less torque was required to move the ankle when the activated brace was worn. There are two possible explanations for this. First, this could be because the natural compressive loads of the ankle are reduced by the distractive force of the brace, which reduces the congruency of the joint and in-turn allows for the joint surfaces to rotate and translate on each other more freely. Alternatively, this shift in values towards the negative could have been due to the brace force vector creating off-axis loads relative to the point of rotation of the Biodex foot plate. In other words, the DAO may have been applying an external dorsiflexor moment due to alignment of the cylinders compared to the Biodex rotational axis, which manifested as a shift in the measured moment values towards the negative. Nevertheless, the resistive moment difference taken at the two extreme ends of motion for each condition was not significantly affected by the brace wear in a clinically relevant manner.

Sagittal plane muscle moments for the ankle during gait were quantified by Sadeghi et al.[80] The peak ankle moment measured during gait was a plantarflexor moment of $1.5 \mathrm{Nm} / \mathrm{kg} \pm 1.20 \mathrm{Nm} / \mathrm{kg}$ at $48 \%$ of the gait cycle. For the individual tested in the present study, the maximum gait ankle moment could be estimated to be approximately $75 \mathrm{Nm}(50 \mathrm{~kg} \mathrm{x} 1.5 \mathrm{Nm} / \mathrm{kg})$. The greatest moment differences were found in the 70 PSI and 80 PSI bracing conditions, where the moment difference was $5.2 \mathrm{Nm}$ and $6 \mathrm{Nm}$, respectively. Compared to the native ankle at $4.5 \mathrm{Nm}$ moment difference, the $1.5 \mathrm{Nm}$ increase in resistive ankle moment only represents $2 \%$ of the maximal moment experienced by the ankle during gait, and it can be concluded that the presence of the DAO does not introduce additional resistance to natural ankle motion.

\section{Conclusion}

Benchtop validation of the DAO provided evidence of the efficacy of the DAO to provide clinically relevant offloading to the user while maintaining a functional range of motion without increasing the effort it takes to move the ankle. The next phase of device evaluation is to assess the functionality of the DAO in a healthy population and in a clinical population with acute and chronic pain of the foot and ankle. 


\section{CHAPTER 4. BIOMECHANICAL COMPARISON OF A NEW DYNAMIC ANKLE ORTHOSIS TO A STANDARD ANKLE-FOOT ORTHOSIS DURING WALKING ${ }^{2}$}

The work in Chapter 4 was preceded by a pilot study comparing the DAO and PTB during over-ground walking (Appendix B). The work in Chapter 4 compared ankle joint kinematics and plantar pressures among the DAO, standard double upright $\mathrm{AFO}$ (DUAFO), and a non-orthosis control (CON) condition in healthy adults during walking. This chapter was published by the ASME Journal of Biomechanical Engineering.

\section{Introduction}

The medical industry has begun delivering non-surgical and pharmaceutical-free treatment options as a means to curtail the recently appreciated elevated use and tragic outcomes of therapeutic opioid abuse.[11,12] Accordingly, research initiatives have emerged to advance the understanding and technology of non-surgical (conservative) treatment options for individuals suffering from pain due to acute and chronic orthopedic conditions. A recent product of this line of research is the Dynamic Ankle Orthosis,[76] which was designed to manage pain of ankle osteoarthritis (OA).

Ankle OA affects over $1 \%$ of the world's population, and between $70-80 \%$ of ankle OA cases result from traumatic injuries (i.e., post-traumatic ankle osteoarthritis).[810] Long-term physical impairments, joint pain, and reduced quality of life are potential outcomes of this condition.[13] Treatment options include invasive surgical procedures such as total ankle replacement and ankle fusion, which are typically performed as a last resort when conservative treatments have been unsuccessful.[13] Currently, the pain associated with OA can be managed using conservative treatment options such as modified footwear and orthotics such as the Ankle-Foot Orthosis (AFO).

The current standard of care AFO for treating OA symptoms is a freelyarticulating leather and metal double upright AFO (DUAFO). This orthosis stabilizes the ankle during gait by limiting ankle motion to the sagittal plane by eliminating frontal plane ankle motion. $[17,18]$. The DUAFO uses a single degree of freedom pinned joint on each side of the ankle to control ankle motion, but the fixed horizontal alignment of the DUAFO joint does not align with the natural, oblique ankle axis.[13] This stabilizing mechanism reduces OA pain, but also prevents natural ankle motion which could worsen OA severity with extended wear.[15,16]

An alternative approach to managing OA pain is to reduce the mechanical loads passing through the joint ("offload" the joint). Reduction of mechanical stress at the ankle

\footnotetext{
${ }^{2}$ Reprinted from final submission with permission. Chung, C. L., DiAngelo, D. J., Powell, D. W., \& Paquette, M. R. (2020). Biomechanical Comparison of a New Dynamic Ankle Orthosis to a Standard Ankle-Foot Orthosis During Walking. J Biomech Eng, 142(5). https://doi.org/10.1115/1.4045549 [78].
} 
during gait is reported to significantly improve OA pain and functional ability.[63-66] For the ankle, however, there are almost no "offloading" orthoses available on the market. The only known weight-bearing orthosis, known as a Patellar Tendon Bearing orthosis (PTB), is designed to reduce axial loads through the ankle and is reported to reduce pain and discomfort associated with ankle OA.[70] The PTB provides offloading of axial forces through the lower leg by $10-50 \%$ bodyweight, but with large variation due to design and fit.[71-75]. The PTB also limits ankle motion in all planes either partially or completely. Ultimately, this orthosis has fallen out of practice for the treatment of ankle OA. Thus, the need remains for a conservative treatment option that preserves ankle motion while offloading the ankle joint to alleviate pain of ankle OA.

The Dynamic Ankle Orthosis (DAO) was recently designed with the intentions of offloading the foot and ankle using a distractive force (i.e., tensile or traction force) and allowing sagittal and frontal plane ankle motion similar to ankle motion in healthy adults.[21,77] The DAO is constructed with three main components: a calf sleeve (for gripping the calf) and a modified shoe (for gripping the foot) connected by a set of two pneumatic cylinders. The pneumatic cylinders attach to the shoe component using ball joints positioned over the oblique anatomical axis of the ankle. The pneumatic cylinders are connected with tubing so that one side can lengthen and the other side can shorten without losing air pressure between the two cylinders. During dynamic tasks such as walking, the pneumatic cylinders work in tandem with the ball joints to enable a functional range of dorsiflexion, plantarflexion, inversion, and eversion of the ankle. When the cylinders are pressurized, a distractive force is generated by the DAO along the axis of the lower leg and is applied through the rotational axis of the ankle. This action creates an offloading effect across the foot and ankle without influencing torque contributions during gait.[77]

The offloading effect is defined as a reduction of the longitudinal loads that pass through the anatomical structures of the foot and ankle complex during stance. The offloaded forces are carried externally through the DAO components to the ground, effectively bypassing the foot and ankle joint. The remaining internal forces (i.e., the bodyweight forces that are not offloaded) are passed through the anatomical structures of the foot and ankle as normal. These internal forces are eventually passed through the plantar aspect of the foot to the shoe and are ultimately absorbed by the ground as well. A benchtop validation of the brace has shown that during quiet stance (i.e., static double legged stance), the DAO offloaded the foot and ankle by up to $30 \%$ of bodyweight and provided minimal resistance to passive ankle motion.[77]

To evaluate the impact of the DAO during gait, the current study compared ankle joint kinematics and peak plantar pressures (PP) among the DAO, standard DUAFO, and a non-orthosis control $(\mathrm{CON})$ condition in healthy adults during treadmill walking. It was hypothesized that DAO would produce sagittal and frontal plane ankle motion comparable to $\mathrm{CON}$ at a set gait speed, and that the DUAFO would restrict frontal plane ankle motion compared to CON. In addition, it was hypothesized that DAO would reduce PP compared to DUAFO and CON, and that DUAFO would not reduce PP compared to CON. 


\section{Methods}

\section{Participants}

A convenience sample of ten healthy young adults (4 male and 6 female; $26.0 \pm$ $3.8 \mathrm{yrs} ; 69.6 \pm 12.7 \mathrm{~kg} ; 1.69 \pm 0.07 \mathrm{~m})$ participated in this study. Participants were free from any chronic disease or orthopedic conditions that could influence walking biomechanics, were free from any lower extremity injury for at least 12 months and were not pregnant. All participants were informed of all procedures and potential risks, and completed written informed consent approved by the institutional review board before testing.

\section{Orthosis Conditions}

At the start of the study, a custom DUAFO and a custom DAO were fabricated for each participant's right limb. All three orthosis conditions (Figure 4-1) used the same shoe model (Model 501, New Balance, USA). The shoes for DAO and DUAFO were each modified identically with a metal stirrup plate embedded in the sole of the shoe for attachment of upper orthosis components. The DAO assembly weighed $1.00 \mathrm{~kg}$, the DUAFO assembly weighed $1.04 \mathrm{~kg}$, and the CON shoe weighed $0.34 \mathrm{~kg}$. The design and alignment of the DAO's rotational axis with the anatomical axis of the ankle joint allowed for at least $10^{\circ}$ of dorsiflexion and $15^{\circ}$ of plantarflexion, and at least $10^{\circ}$ of inversion and eversion.[77]

To determine a "targeted offloading" level for this study, a group of orthotists were consulted regarding level of offloading necessary to achieve pain reduction in OA patients. A targeted offloading of up to $10 \%$ bodyweight was recommended. Using this recommendation along with the precedent offloading provided by the PTB (reportedly offloaded $10-50 \%$ of the user's bodyweight),[71-75] the DAO was tuned to offload a targeted value of $10 \%$ bodyweight for each participant using the static protocol described as follows.

\section{Experimental Protocol}

Three randomized orthosis conditions were compared: 1) non-orthosis (CON), 2) DAO, and 3) DUAFO (Figure 4-1). Pressure insoles (100 Hz Pedar, Novel Electronics, MN, USA) were used to collect PP under the foot during treadmill walking. The pressure insoles were located in between the foot and the interior padding of the shoe for all three orthosis conditions. The pressure insoles were zeroed using a standard procedure for all three orthosis conditions. For the DAO condition, static offloading (with targeted value of $10 \%$ bodyweight) was calculated in quiet stance by taking the percent difference in the insole PP readings before and after cylinder inflation. This static procedure was performed five times per participant. Each orthosis was worn for approximately 10 
A)

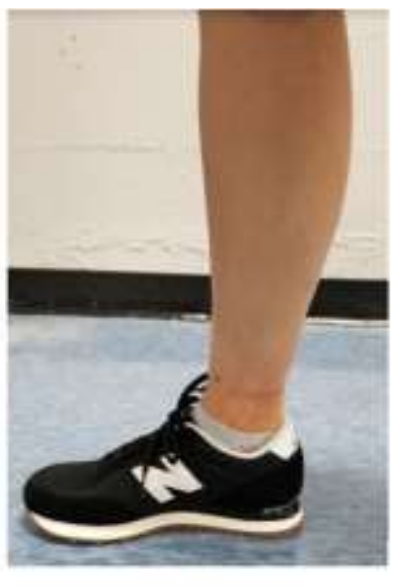

B)
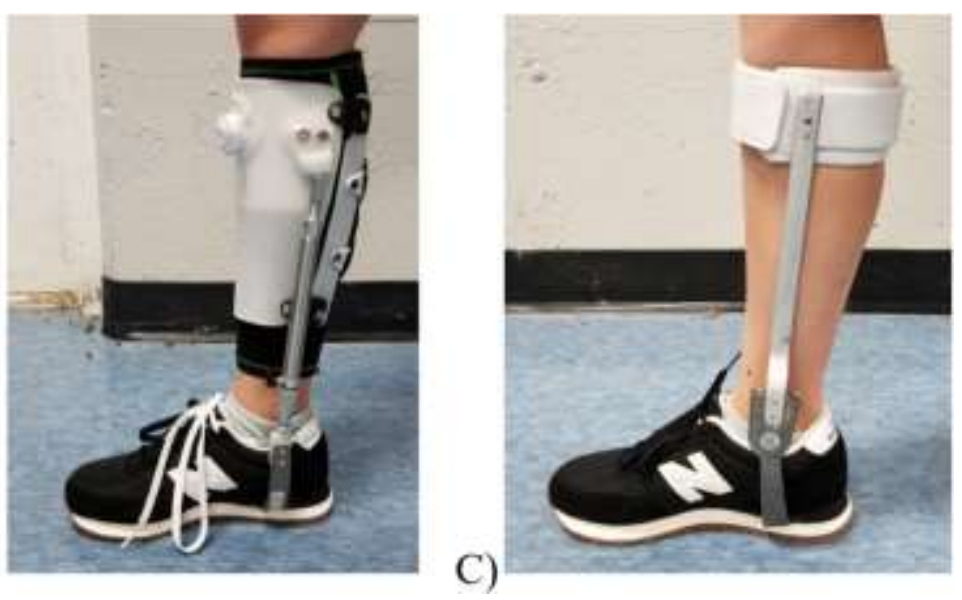

Figure 4-1. Orthosis Configurations

A) Non-Orthosis Control (CON), B) Dynamic Ankle Orthosis (DAO), and (C) Double Upright Ankle-Foot Orthosis (DUAFO). 
minutes (standing and walking) before testing to provide a period of familiarization. Participants walked over a $25 \mathrm{~m}$ tiled laboratory walkway at a self-selected speed. Preferred walking speed was measured and controlled during over-ground walking using an electronic timer (54035A, Lafayette Instruments Inc., USA) and two infrared photocells (63501 IR Lafayette Instruments Inc., USA), spaced 3 meters apart. The two photocells were placed facing the walkway. As the subject walked along the walkway, they crossed the infrared beam of one photo-cell, signaling the start of the timer, and then they crossed the infrared beam of the second photo-cell, signaling the stop of the timer. The time elapsed and the known distance between the photo-cells were used to compute walking velocity

A 9-camera 3D motion capture system (240 Hz, Qualisys AB, Sweden) was used to obtain three-dimensional (3D) position data during treadmill walking. Individual spherical markers were secured over the iliac crests, greater trochanters, medial and lateral femoral epicondyles, medial and lateral malleoli, and first and fifth metatarsal heads. Marker clusters of three or four tracking non-collinear markers rigidly attached to thermoplastic plates were secured to the pelvis and to the right thigh, shank, and foot. The marker placement on the shank and foot was modified for the DAO condition due to placement restrictions. The modified marker placement involved shifting the shank markers onto the rigid anterior portion of the DAO and the foot markers onto the dorsal aspect of the shoe. Following a one-second static trial, anatomical markers were removed, and treadmill walking began. Participants walked on a treadmill (Excite+ Run Now 900, TechnoGym, USA) [81-86] at $1.4 \mathrm{~m} / \mathrm{s}$ [87,88] for at least two minutes prior to data collection. 3D position data and PP were captured for ten sequential steps. Participants took a forceful step on the treadmill at the start of each collection period to synchronize the motion capture and PP systems. Participants rested for 5-6 min between orthosis condition testing procedures to avoid fatigue. Participants rated the levels of discomfort using custom visual analog scale (VAS) survey after walking in each orthosis. Discomfort was rated with 0 : "very comfortable," 2: "somewhat comfortable,"4: "uncomfortable," 6: "moderately uncomfortable," and 10: "extremely uncomfortable." See Appendix C for extended methodology (Figures C-1 - C-5).

\section{Data Analysis}

Three-dimensional kinematic data were processed and analyzed using Visual3D (C-Motion, USA). The raw kinematic data were interpolated to fill gaps no longer than 10 frames and filtered using a Butterworth low-pass filter with a $6 \mathrm{~Hz}$ cutoff frequency. A right-hand rule with a Cardan rotational sequence (X-y-z) was used for 3D angular computations where $\mathrm{X}$ represents rotations about the mediolateral axis of the proximal segment, and $y$ and $\mathrm{z}$ represent rotations about the anteroposterior and vertical axes of the distal segment, respectively. Sagittal and frontal plane ankle angular kinematics were resolved in the shank coordinate system and all variables were analyzed during the stance phase of gait. A velocity-based algorithm was used to define heel strike and toe-off.[89] Early stance was defined as the first $25 \%$ of stance phase (i.e. Load Response).[25] During treadmill walking at a set speed, stance time, step length, and cadence were all 
measured during walking. Stance time was calculated as the time between heel strike and toe-off, step length was calculated as the antero-posterior distance between two consecutive steps, and cadence represented the number of steps per minute.

Peak PP captured during treadmill walking under seven specific areas were analyzed using pedar/S (Standard) software (Novel Electronics, MN, USA). Peak PP represented the highest pressure recorded by pressure insole sensors of each area of interest during the stance phase of gait. The plantar areas included the medial heel, lateral heel, midfoot, medial forefoot, lateral forefoot, hallux, and toes 2-5. The amount of offloading provided by each orthosis during walking was calculated for each plantar region using Equation 4-1.

$$
\% \text { Offloading }=\left(1-\frac{P P_{\text {Orthosis }}}{P P_{\text {CON }}}\right) \times 100 \%
$$

\section{Statistical Analysis}

A Shapiro-Wilk Test was used to determine normality of the data per condition for each dependent variable. Repeated measures ANOVA were used to determine the within-subject orthosis effect for all dependent variables (SPSS 24.0, IBM). Mauchly's Test was used to confirm the ANOVA assumption of sphericity. If the test failed $(p<0.05)$, the Greenhouse-Geisser correction was applied to alter degrees of freedom of the ANOVA and produce an F-ratio where the Type I error rate was reduced. When a significant F-test was observed, paired t-tests were used to compare means among orthosis conditions. When the assumption of normality was violated (i.e., nonparametric), an independent samples Kruskal Wallis Test with pairwise comparisons was performed to determine the within-subject orthosis effect. Post hoc pairwise analyses were performed with the Least Significant Difference (LSD) method for multiple comparisons. A significance level of $\mathrm{p}<0.05$ was set a priori for all tests. Cohen's $d$ effect sizes were also calculated to assess the magnitude of dependent variable mean differences (i.e., small: $d<0.2$, moderate: $0.2 \leq d<0.8$; large: $d \geq 0.8$ )

\section{Results}

Orthosis effects were observed for peak PP under hallux and toes 2-5 (Table 4-1). Peak PP under hallux in the DAO were smaller than the CON $(d=0.98)$ and the DUAFO $(d=1.26)$. Peak PP under toes $2-5$ were moderately smaller in the DAO than the DUAFO $(d=0.65)$, but greater in the DUAFO compared to CON $(d=0.35)$. Peak PP under other plantar areas were not statistically different among orthosis conditions.

The ensemble curves for sagittal and frontal plane ankle kinematics are shown in Figures 4-2 and 4-3, respectively. Orthosis effects were observed for early stance peak plantarflexion angular velocity, eversion ROM, and early stance peak eversion angular 
Table 4-1. Peak Plantar Pressures for Each Plantar Area in Each Orthosis Condition During Set Speed Treadmill Walking

\begin{tabular}{lcccc}
\hline Plantar Area & CON & DAO & DUAFO & p-Value \\
\hline Medial Heel $(\mathrm{kPa})$ & $195.5 \pm 48.1$ & $167.0 \pm 25.4$ & $198.2 \pm 54.3$ & 0.377 \\
Lateral Heel $(\mathrm{kPa})$ & $198.5 \pm 57.9$ & $173.4 \pm 45.3$ & $190.2 \pm 50.2$ & 0.334 \\
Midfoot $(\mathrm{kPa})$ & $106.5 \pm 27.5$ & $110.7 \pm 43.6$ & $100.6 \pm 38.3$ & 0.703 \\
Medial Forefoot $(\mathrm{kPa})$ & $222.2 \pm 44.9$ & $222.9 \pm 74.7$ & $204.6 \pm 45.6$ & 0.338 \\
Lateral Forefoot $(\mathrm{kPa})^{192.4 \pm 37.2}$ & $189.0 \pm 34.9$ & $191.5 \pm 39.2$ & 0.513 \\
Hallux $(\mathrm{kPa})^{*}$ & $235.2 \pm 61.9$ & $179.6 \pm 51.5^{\mathrm{a}, \mathrm{c}}$ & $249.6 \pm 59.4$ & $<0.001$ \\
$2^{\text {nd }}-5^{\text {th }}$ Toes $(\mathrm{kPa})$ & $148.1 \pm 51.9$ & $131.0 \pm 48.6^{\mathrm{c}}$ & $168.5 \pm 65.3^{\text {a }}$ & 0.018 \\
\hline
\end{tabular}

Notes: Mean $\pm \mathrm{SD} ;{ }^{*}$ : orthosis effect. ${ }^{\mathrm{a}}$ : significant difference from $\mathrm{CON} ;{ }^{\mathrm{b}}$ : significant difference from DAO; ${ }^{c}$ : significant difference from DUAFO. Significance: $p<0.05$.

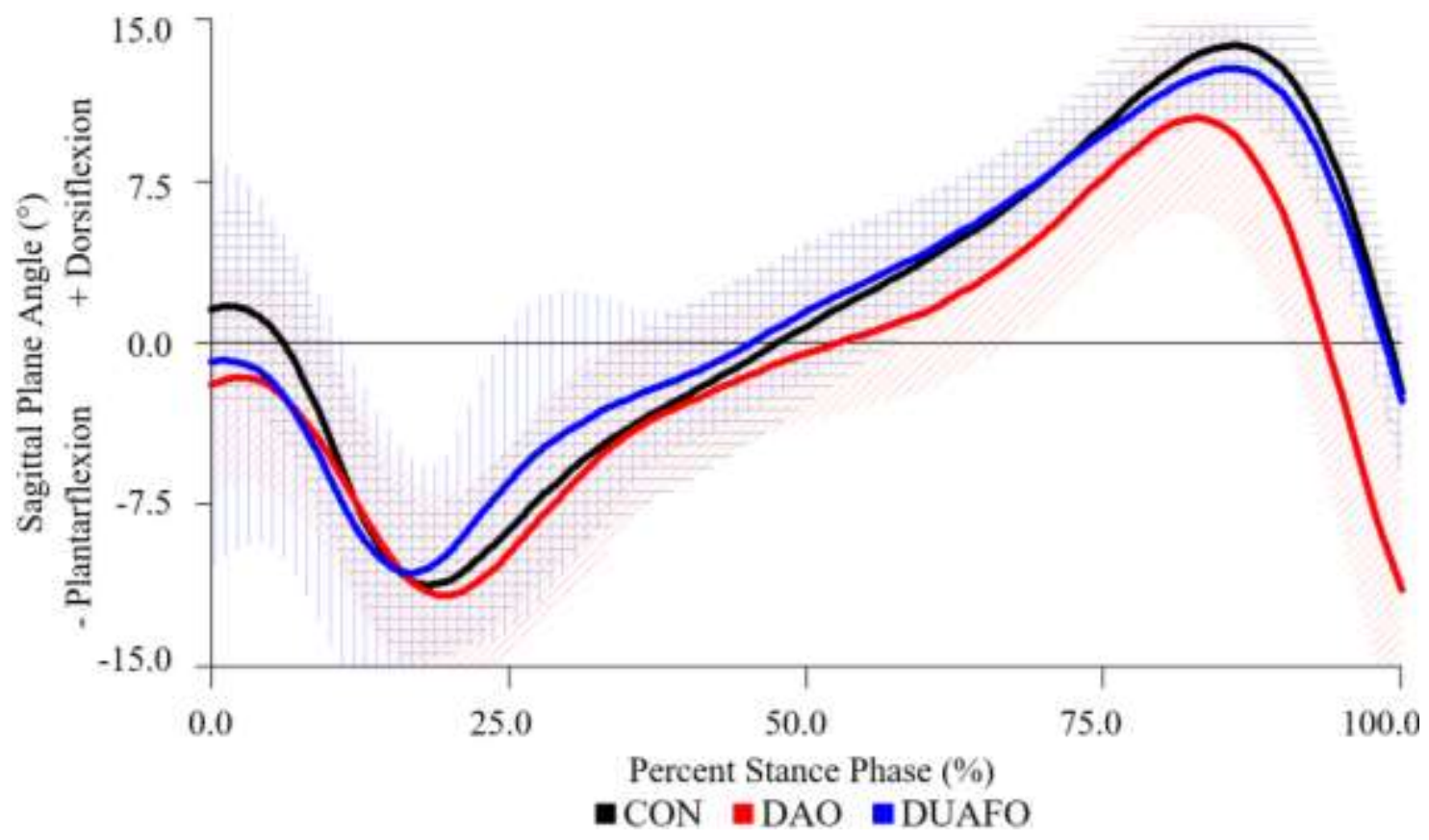

Figure 4-2. Sagittal Plane Ankle Joint Angle Ensemble Curves for the Right Limb of All Three Orthosis Conditions

Mean $\pm \mathrm{SD}$; Control (CON), Dynamic Ankle Orthosis (DAO), and Double Upright Ankle-Foot Orthosis (DUAFO). 


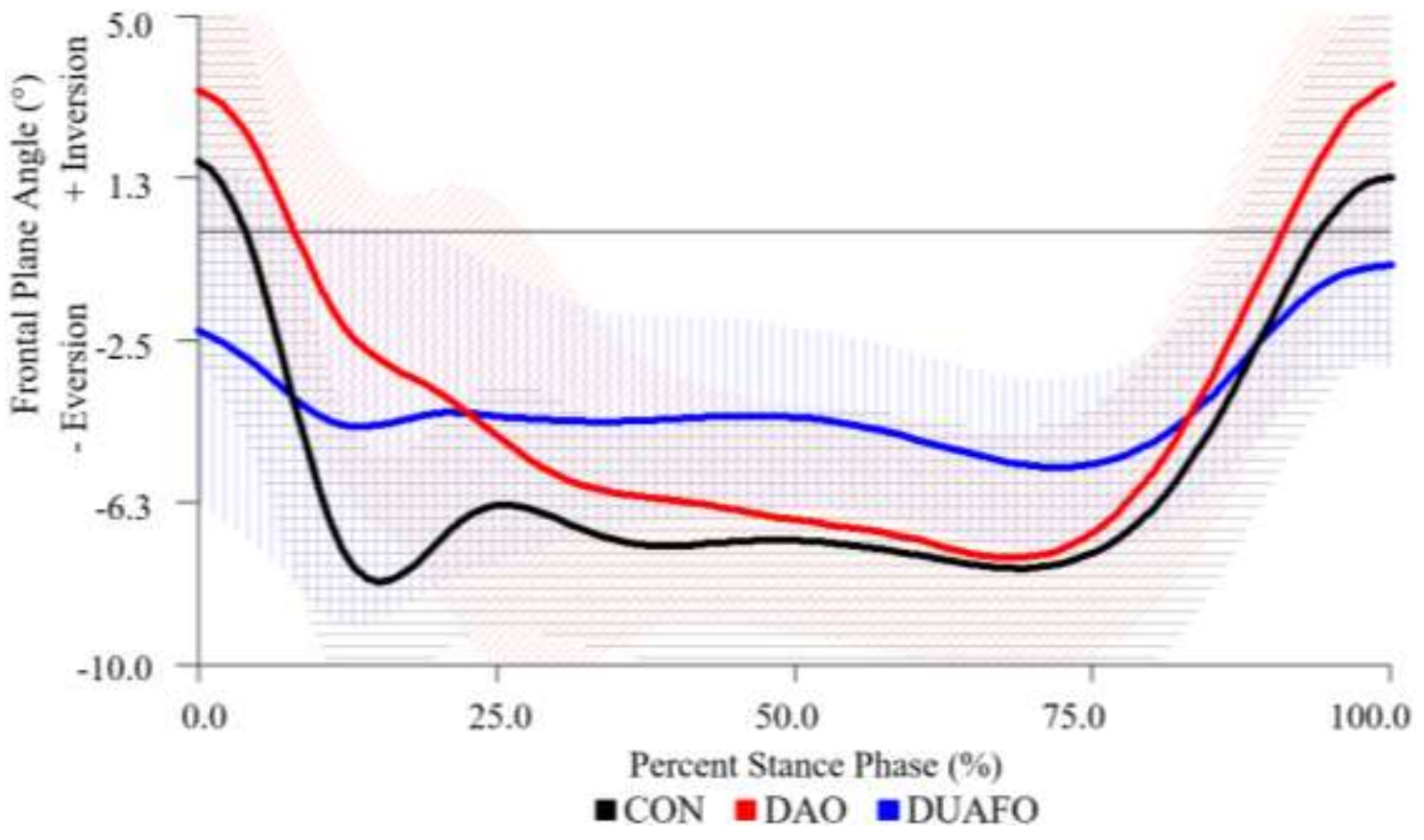

Figure 4-3. Frontal Plane Ankle Joint Angle Ensemble Curves for the Right Limb of All Three Orthosis Conditions

Mean $\pm \mathrm{SD}$; Control $(\mathrm{CON})$, Dynamic Ankle Orthosis (DAO), and Double Upright Ankle-Foot Orthosis (DUAFO). 
velocity (Table 4-2). Early stance peak plantarflexion angular velocity was smaller in the DAO compared to both CON $(d=1.51)$ and DUAFO $(d=1.13)$. The DAO showed similar peak eversion $(d=0.02)$ and eversion ROM $(d=0.35)$ compared to CON. Eversion ROM was much smaller in the DUAFO compared to both CON $(d=2.64)$ and DAO $(d=2.93)$. Finally, early stance peak eversion angular velocity was smaller in the DAO $(d=1.21)$ and much smaller in the DUAFO $(d=2.79)$ compared to CON.

An orthosis effect was observed for stance time during treadmill walking with a longer stance time on the right limb in the DAO compared to both CON $(d=1.96)$ and DUAFO $(d=1.57)$ (Table 4-3). No orthosis effects were observed for preferred speed during over-ground level walking (Table 4-3). No orthosis effects were observed for step length and cadence during treadmill walking (Table 4-3).

The DAO was found to be more uncomfortable than the CON during standing ( 3 \pm 2 vs $<1 \pm 2 ; \mathrm{p}=0.003 ; d=1.17)$ and walking $(5 \pm 2 \mathrm{vs}<1 \pm 1 ; \mathrm{p}<0.001 ; d=2.87)$. The discomfort scores for DUAFO were not statistically different than CON during standing $(1 \pm 1 ; \mathrm{p}=0.276 ; d=0.48)$ or walking $(3 \pm 2 ; \mathrm{p}=0.101)$ although a large effect size $(d=1.43)$ suggests that DUAFO was more uncomfortable than CON during walking. See Appendix D for extended results (Figures D-1 - D-3 and Tables D-1 - D-3).

\section{Discussion}

The present study compared ankle kinematics and PP among the DAO, standard DUAFO, and a non-orthosis control $(\mathrm{CON})$ in healthy adults during treadmill walking. Our hypothesis that the DAO would produce ankle kinematics similar to CON was partially supported. The DAO reduced plantarflexion angular velocity in early stance compared to CON and DUAFO. In addition to a longer stance time, although not statistically different, the DAO produced a more plantarflexed ankle position and a slightly smaller early stance plantarflexion ROM especially compared to CON $(d=0.77$ and $d=1.00$, respectively) (Table 4-2). These differences in the DAO contributed to the slower peak plantarflexion velocity compared to CON. Articulating AFO generally do not affect sagittal plane motion [17] and therefore, the similar sagittal plane angles at foot strike and in early stance are unsurprising. In the frontal plane, our hypothesis was supported. The DAO permitted similar peak eversion angles and eversion ROM compared to CON while eversion ROM was greatly reduced in the DUAFO compared to CON and DAO. By limiting frontal plane ankle motion, the primary function of the DUAFO is to manage symptoms in clinical populations. However, the more natural frontal plane ankle motion in the DAO could help slow progression of degenerative foot and ankle diseases like ankle OA while the limited frontal plane motion of the DUAFO could exacerbate OA severity with extended wear.[15,16]. Further, the DAO produced slower peak ankle eversion velocity compared to CON which could be beneficial for some conditions that lead to compromised ankle stability. This finding was not unique to the DAO as the DUAFO also produced slower peak ankle eversion velocity compared to CON. Therefore, this ankle eversion velocity difference between DAO and CON does not 
Table 4-2. $\quad$ Ankle Joint Kinematics in Each Orthosis Condition During Set Speed Treadmill Walking

\begin{tabular}{lcccc}
\hline Variables & CON & DAO & DUAFO & p-Value \\
\hline Sagittal Angle at FS $\left(^{\circ}\right)$ & $1.5 \pm 4.3$ & $-2.2 \pm 5.7$ & $-0.5 \pm 8.0$ & 0.217 \\
Early Stance PF ROM $\left(^{\circ}\right)$ & $12.9 \pm 2.1$ & $9.7 \pm 4.3$ & $11.5 \pm 2.7$ & 0.066 \\
Early Stance Peak PF $\left(^{\circ}\right)$ & $-11.3 \pm 3.6$ & $-11.9 \pm 4.1$ & $-12.0 \pm 6.5$ & 0.951 \\
DF ROM $\left(^{\circ}\right)$ & $24.8 \pm 4.0$ & $23.0 \pm 3.3$ & $24.8 \pm 6.8$ & 0.414 \\
Early Stance Peak PF Angular Velocity $\left({ }^{\circ} \cdot \mathrm{s}^{-1}\right)^{*}$ & $-201.1 \pm 31.8$ & $-138.1 \pm 49.8^{\text {a,c }}$ & $-188.5 \pm 38.6$ & $<0.001$ \\
Peak EV $\left(^{\circ}\right)$ & $-9.4 \pm 2.8$ & $-9.3 \pm 3.4$ & $-7.0 \pm 1.9$ & 0.103 \\
EV ROM $\left(^{\circ}\right) *$ & $11.0 \pm 2.8$ & $12.1 \pm 3.0$ & $4.6 \pm 2.0$ & $<0.001$ \\
Early Stance Peak EV Angular Velocity $\left({ }^{\circ} \cdot \mathrm{s}^{-1}\right)^{*}$ & $-171.5 \pm 49.9$ & $-112.2 \pm 48.3{ }^{\mathrm{a}}$ & $-59.5 \pm 27.1$ & $<$ a,b $<0.001$ \\
\hline
\end{tabular}

Notes: Mean $\pm \mathrm{SD} ;{ }^{*}$ : orthosis effect; ${ }^{\text {a: }}$ significant difference from $\mathrm{CON} ;{ }^{\mathrm{b}}$ : significant difference from DAO; ${ }^{\mathrm{c}}$ : significant difference from DUAFO. Significance: p<0.05. PF: plantarflexion; DF: dorsiflexion; EV: eversion; FS: foot strike; ROM: range of motion. 
Table 4-3. $\quad$ Preferred Speed During Over-Ground Walking and Spatio-Temporal Variables in Each Orthosis Condition During Set Speed Treadmill Walking

\begin{tabular}{lcccc}
\hline Variables & CON & DAO & DUAFO & p-Value \\
\hline Preferred Speed $\left(\mathrm{m} \cdot \mathrm{s}^{-1}\right)$ & $1.20 \pm 0.14$ & $1.12 \pm 0.14$ & $1.17 \pm 0.13$ & 0.288 \\
Stance Time (s) ${ }^{*}$ & $0.66 \pm 0.02$ & $0.71 \pm 0.03^{\mathrm{a}, \mathrm{c}}$ & $0.67 \pm 0.02$ & $<0.001$ \\
Step Length (m) & $0.75 \pm 0.03$ & $0.75 \pm 0.03$ & $0.76 \pm 0.03$ & 0.196 \\
Cadence (steps $\left.\cdot \mathrm{min}^{-1}\right)$ & $114 \pm 5$ & $112 \pm 5$ & $111 \pm 3$ & 0.134 \\
\hline
\end{tabular}

Notes: Mean $\pm \mathrm{SD} ;{ }^{*}$ : orthosis effect; ${ }^{\mathrm{a}}$ : significant difference from $\mathrm{CON} ;{ }^{\text {b}}$ : significant difference from DAO; ${ }^{c}$ : significant difference from DUAFO. Significance: $p<0.05$. 
fully address this limitation of the currently available DUAFO orthosis. However, the current findings generally support the notion of "more natural" ankle motion in the DAO compared to the current standard of care DUAFO.

Our second hypothesis was partially supported as peak PP were significantly different among orthosis conditions under the hallux and toes 2-5. The DAO led to large reductions in PP under the hallux compared to CON and DUAFO, and large reductions in PP under toes 2-5 compared to DUAFO only. Additionally, the DUAFO increased PP under toes 2-5 compared to CON. Specifically, the DAO offloaded the toes $2-5$ by $12 \%$ and the hallux by $24 \%$ compared to CON while the DUAFO created a $14 \%$ and $6 \%$ increase in the peak PP of toes 2-5 and hallux, respectively. Though a statistical orthosis effect was not found, a moderate reduction of peak PP was observed at the medial heel $(d$ $=0.74,15 \%)$ and lateral heel $(d=0.48,13 \%)$ with the DAO compared to the CON, and the midfoot and forefoot PP remained the same. Since orthotists recommended a targeted offloading level of $10 \%$ for pain relief of OA, it is reasonable to believe that offloading levels of $13-15 \%$ in the heel represent a "clinically relevant" benefit of the DAO.

The reduction in peak PP under hallux and toes in the DAO occurred in parallel with a longer stance time. Lower peak PP with a longer contact time has also been observed while wearing a pneumatic walking orthosis designed to reduce occurrence of neuropathic plantar foot ulcers compared to a regular shoe.[90] Reduced PP under the forefoot with increases in heel PP have been observed while using pneumatic walking orthosis during treadmill walking.[91] Contrarily, PTB orthoses, designed with varying levels of heel clearance to completely unweigh the heel, have been shown to reduce hindfoot peak PP and increase forefoot PP compared to a regular shoe.[71] These findings suggest that there exists a trade-off in orthosis offloading of the foot: forefoot offloading can be achieved at the expense of the heel. Following this load shift principle, the DAO might be restricted to only significantly offloading one area of the foot (i.e., heel or toes), which might explain why a significant orthosis effect was not found for peak heel PP as expected.

Preferred over-ground walking speed and discomfort were also compared while wearing each orthosis. Preferred over-ground walking speed was not different among orthoses, but participants rated the DAO to be more uncomfortable than CON and DUAFO during both standing and walking while the DUAFO was only more uncomfortable than CON during walking. Rigid AFO have been shown to reduce walking velocity, step length, and step time in healthy individuals, but similar to our current findings, dynamic AFOs do not generally affect these walking parameters.[92] In addition to the greater discomfort rating, participants also commonly reported tingling sensations in the lower leg and foot towards the end of testing when wearing the DAO. It is possible that the DAO was too tight around the calf increasing pressure and restricting blood flow. The set testing speed of the present study $\left(1.4 \mathrm{~m} \cdot \mathrm{s}^{-1}\right)$ was greater than the measured preferred speed for all three orthosis conditions and likely required greater effort while walking at the set speed in both orthoses. This may have contributed to the increased discomfort while wearing the DAO as well as the increased stance time in the DAO compared to CON and DUAFO. The addition of a mass (1.8 to $4.6 \mathrm{~kg}$ ) to the distal 
end of the leg of an able-bodied individual has been shown to cause altered walking posture in treadmill walking.[93,94]. The added mass of the orthoses may have also contributed to greater discomfort in the DAO and DUAFO (walking only) but the added mass was relatively small (i.e., $0.66-0.7 \mathrm{~kg}$ ), and was the same for both orthoses. Further, during the swing phase, DAO activation (cylinders inflated to desired pressure) pushed the shoe down which would lead the shoe to slip off if participants did not actively dorsiflex their ankle and flex their toes. This "gripping" action may have also contributed to the discomfort perceived while wearing the DAO. Finally, although the DAO allows for more natural ankle motion and offloads the forefoot during walking, high rated discomfort while wearing the DAO will require design alterations of future versions to improve user comfort.

This study had certain limitations. The small sample size makes the results difficult to generalize to larger population and the orthosis effects may be different in other populations. However, the decision not to expand the sample size was made after the research team concluded that the current findings were sufficient to justify a larger study on DAO wear in a clinical population following some design improvements. The primary purpose of the present study was to demonstrate that the DAO did not greatly alter the kinematic range of ankle motion of the user during walking. The data from the first 10 participants verified that the DAO did not interfere with walking kinematics in healthy individuals and supported a shift to a clinical population. The secondary purpose of the present study was to demonstrate that the DAO could maintain offloading during a dynamic task such as walking. The convenience sample of 10 subjects caused some PP results to be underpowered, which helps explain why statistical orthosis effects in the PP variables at the heel were not found as expected. A power analysis showed that over 45 subjects would be necessary to show statistical significance in the peak plantar pressure variables at the heel. Since extensive benchtop testing has already proven the capacity of the DAO to offload the lower limb in quiet stance,[77] the research team did not think it necessary to expand the study sample size beyond the initial 10 participants to prove this secondary point in a population of healthy individuals. The next phase of this research will include combined measures of offloading and pain scores during walking and other functional tasks in a clinical population with mechanical foot and ankle pain. In addition to these limitations due to sample size, our current findings only addressed acute difference since participants had not worn these orthoses regularly. Thus, the chronic use effects of these orthoses may alter our current findings. Testing was performed during treadmill walking which may have created an artificial walking environment compared to over ground walking, although gait kinematics should not have been affected.[81-87] Treadmill walking was chosen as the walking modality to validly control walking speed during testing. The 10 minutes of acclimation time was also a limitation. Although 10 minutes was enough time for some individuals to get used to the orthosis, it may have not been enough time for all of the study participants. In a clinical setting, the orthosis fitting procedures permit plenty of time to acclimate to the orthosis and to make modifications for comfort. Due to the laboratory setting, adjustments for comfort were limited to what could be provided without altering the construct of the orthosis itself. 
Finally, using only the plantar pressure variable to evaluate offloading of the foot and ankle could be considered a limitation of the study. The DAO was developed as a novel conservative treatment option designed to "offload the ankle joint" specifically for cases of ankle osteoarthritis. However, the DAO serves to offload the entire braced segment (i.e., the anatomical structures between the calf sleeve and the shoe including the foot and ankle). It was assumed that the loads transmitted through the ankle joint must also pass through the structures of the foot and eventually pass through the plantar surface of the foot to the inner shoe sole material. Therefore, the influence of the DAO on the bodyweight loads passing through the ankle were measured under the foot, specifically between plantar surface of the foot and the inside of the shoe. The peak plantar pressure variable has been used widely to demonstrate the effect of AFO and foot orthotic interventions during dynamic tasks. To measure the offloading effect of the DAO during gait, instrumented pressure insoles were used to capture changes in peak pressures between the foot and the shoe. By comparing peak plantar pressure changes between each of the bracing conditions, it was reasoned that the ankle was also being offloaded to some degree. For a stronger evaluation of the impact of this device on plantar loading during gait, future studies should include other loading variables such as the mean pressure. At the time of this study, the only validated tools available for this measurement were the pressure insoles. Recent advancements in this area of technology now enable "in-shoe" measurements of plantar forces, loading rate, and impulse variables, and will be included in future research assessing the DAO.

\section{Conclusion}

This study demonstrates the capacity of the DAO to provide a clinically relevant level of offloading of mechanical loads passing through the ankle and foot during ambulation without greatly affecting sagittal plane and frontal plane ankle motion and gait parameters compared to CON. The present study also quantified the shortcomings of the standard DUAFO and current DAO design. The findings and feedback received during this study will be used to improve DAO fit for better comfort and load attenuation. Future work will assess the efficacy of the DAO in clinical populations with mechanical foot and ankle pain. The findings of this future work will yield novel insight into the association between plantar loading, foot and ankle pain relief, and improved joint function. With extended DAO wear, patients may regain lost foot and ankle function. Not only will this have long term positive effects on their overall health and quality of life, it could eliminate the need for pharmaceutical interventions and delay the need for joint fusion or replacement surgery. 


\section{CHAPTER 5. IMPACT OF THE DYNAMIC ANKLE ORTHOSIS ON MECHANICAL PAIN AND FUNCTION IN PATIENTS WITH FOOT AND ANKLE DISEASES}

This chapter presents a clinical evaluation of the DAO. The purpose of this study was to evaluate the acute effects of the DAO on pain levels, under-foot loading, and function in individuals with mechanical foot and ankle pain.

\section{Introduction}

Over two million Americans visit the doctor each year for foot and ankle pain due to a degenerative condition or injury.[1] Prevalence estimates for foot and ankle pain vary from $9 \%$ to $36 \%$ due to inconsistency in how "foot and ankle pain" is defined.[2] Mechanical pain (i.e., pain caused by weight-bearing activities and joint motion) of the foot and ankle often stems from structural damage related to conditions such as a contusion, sprain, fracture, osteoarthritis (OA), tendinopathy, synovitis, and deformities. $[8,95,96]$ In general, pain of the foot and ankle is associated with obesity and increased age, [2,7] but it is also known to impact individuals of all ages for a variety of reasons. Traumatic injuries are the principal cause of developing degenerative orthopedic conditions of the foot and ankle like OA (70-80\% post-traumatic, PTOA),[96] which typically presents in adults ( $>55$ years old) as joint stiffness, inflammation, swelling, reduced range of motion, and pain exacerbated with increased activity.[8,9,14] One in five middle-aged adults ( $\geq 45$ years old) reports general foot and ankle pain, with twothirds reporting moderate or worsening impairment with daily activities.[7,35] Older adults ( $\geq 65$ years old) are affected by foot and ankle pain the most, with $30.9 \%$ experiencing acute tenderness of the foot and ankle and $14.9 \%$ report reoccurring pain of the foot and ankle.[36]

Lower limb joint pain can have a significant detrimental impact on health-related quality of life due to reduced functional abilities (i.e., walking, being active, etc.).[6,8-13] Treatments for severe ankle pain include surgical interventions such as ankle replacement (arthroplasty) and ankle fusion (arthrodesis). However, these surgical procedures create a significant economic burden with over 80,000 ankle fusions and 16,000 ankle replacements performed annually.[33,34,42,43] Surgical procedures are usually only considered after conservative treatment options fail. Common conservative treatment options for mild to moderate cases of mechanical foot and ankle pain include the prescription of custom shoe orthotics, bracing, physical therapy, and medication.[11] An Ankle-Foot Orthosis (AFO) and Ankle Stabilizing Orthosis (ASO) reduce joint motion to effectively manage pain symptoms.[14-16] Simply bracing the foot and ankle with traditional orthoses, however, falls short of fully addressing the foot and ankle functional impairments. Since a standard brace limits motion at the ankle, joint function is sacrificed for the benefit of pain relief.[17,18] Altered gait patterns due to the restrictive nature of current practice braces may lead to disease progression and eventually, require surgery. Another limitation with current bracing practice is the inability of the brace to reduce the 
mechanical loads of a joint. A $10-20 \%$ reduction in bodyweight can reduce pain and improve function and quality of life in individuals with OA.[49-52] Rehabilitation techniques such as hydrotherapy and unloading treadmills have been used to reduce lower extremity loading and improve function, but options can be difficult to access.[5358] For the foot and ankle, however, the only existing treatment option that utilizes an offloading strategy is a surgical technique called ankle arthrodiastasis,[63] which reduces the natural compression of the foot and ankle joints using an external fixator device to apply traction across the segments.[14,63-66] Reducing mechanical loads at the foot and ankle by diverting the longitudinal loads passing through the joint ("offload") with an external device (i.e., orthosis) could have positive outcomes on symptoms and function in those with foot and ankle pain.

The Dynamic Ankle Orthosis (DAO) was designed to manage mechanical pain and restore function to individuals suffering from orthopedic foot and ankle conditions. The DAO is the first AFO to provide the coupled benefits of foot and ankle offloading while allowing natural talocrural and subtalar joint motion during level-walking. [77,78] The DAO consists of a calf sleeve and a modified shoe (Figure 5-1) connected by pneumatic (air) cylinders that apply a controlled distractive force across the ankle when inflated. Anatomically positioned tie rod ball joints located in line with the center of the malleoli permit functional ankle motion. When activated (i.e., pressurized), the DAO offloads longitudinal forces passing through the foot and ankle by up to $30.5 \%$ in quiet stance without changing the rotational effort required to move the ankle through a functional range of motion.[77] Finally, the DAO reduces peak plantar pressures under the braced foot by up to $24 \%$ while permitting frontal and sagittal plane ankle motions similar to those during unbraced walking compared to a standard of care AFO in healthy young adults.[78] Although the DAO can reduce plantar loads and allow natural ankle motion in healthy adults, its effectiveness in providing the offloading benefits and improving symptoms and function in patients with foot and ankle pain has not yet been assessed. Therefore, the purpose of this study was to assess the effects of acute DAO wear on perceived function and underfoot force offloading in patients with foot and ankle pain who experienced different changes in mechanical foot and ankle pain while wearing the DAO. We hypothesized that acute DAO wear would reduce underfoot mechanical loads and improve perceived pain symptoms and function during activities of daily living compared to an unbraced control condition. We also hypothesized that patients would notice improvement in their overall pain symptoms and ability to perform activities ("function") with DAO wear.

\section{Methods}

\section{Participants}

Twenty-five patients were enrolled in the study ( 8 male / 17 female, $42.6 \pm 14.1$ years old, $94.6 \pm 21 \mathrm{~kg}, 170.1 \pm 8.6 \mathrm{~cm}$ ) according to inclusion and exclusion criteria as per recommendation by their foot and ankle surgeon. To be included in the study, the 


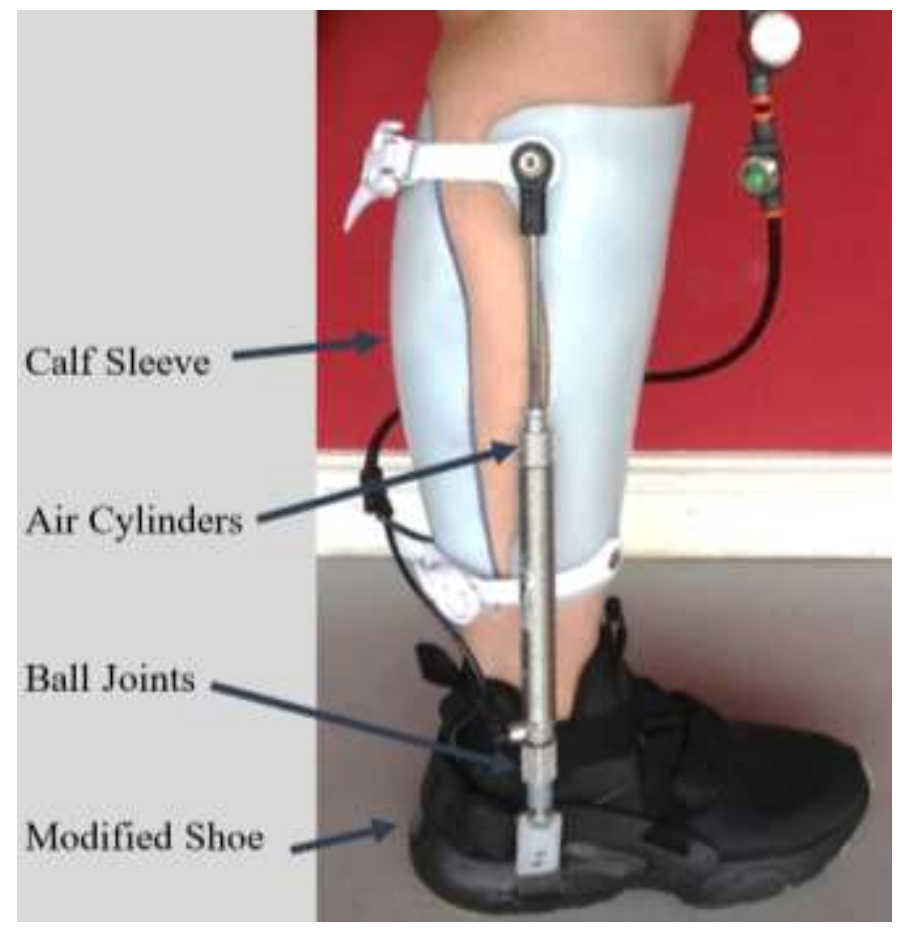

Figure 5-1. DAO Components

The upper and lower DAO components (calf sleeve and modified shoe) grip the anatomy of the lower limb. These two sections are connected by pneumatic (air) cylinders, which apply a controlled distractive force across the ankle joint when inflated, and anatomically positioned tie rod ball joints, which permit functional ankle motion. 
patients had to have been between 18 and 65 years old with a bodyweight not exceeding $300 \mathrm{lbs}$. The targeted population had a right foot or ankle diagnosis and were symptomatic with mechanical pain. Participants were ambulatory, although most claimed to be functionally impaired to some degree by their condition (i.e., can only walk for a limited time or distance). Patients with a history of prior ankle fusion or peripheral vascular disease were excluded from the study. During the enrollment process, patients were informed of all study procedures and consented using a document approved by the Institutional Review Board for human participant research.

\section{Study Procedures}

All study procedures were performed in a clinic setting under the supervision of orthopedic surgeons and/or physical therapists. Before collecting data, information was obtained from the patient's medical record including their age, height, weight, diagnosis, and prescribed treatment plan. A Foot \& Ankle Disability Index (FADI) survey was administered to quantify the participant's level of disability.[97,98] Participants then performed a series of functional activities (Table 5-1). These functional activities were defined in consultation with physical therapists and foot and ankle orthopedic surgeons because they are common tasks used by clinicians during traditional functional assessments. [99-102] The activities were performed sequentially in the order listed for all participants with short rest periods between activities (typically $<1$ minute). Participants completed the functional activities without and with the brace. The order of the conditions tested (DAO, CON) was randomized to reduce treatment bias, and as a result, 14 individuals wore CON first and 11 wore DAO first. Participants could rest for up to 10 minutes between the first and second series of activities while the bracing condition was changed.

A standard Visual Analog Scale (VAS) was used to quantify foot or ankle pain immediately following each functional activity. It was explained to each participant that a score of 0 represented "no pain" and a score of 10 represented "emergency room visit." Further, validated [103-105] instrumented force insoles (force range: $0-2550 \mathrm{~N}$, frequency: $200 \mathrm{~Hz}$, resolution: $10 \mathrm{~N}$, calibrated accuracy: $\pm 5 \%$, loadsol ${ }^{\mathrm{TM}}$, Novel, Inc., St. Paul, MN, USA) were used to measure peak vertical force between the foot and the insole of the shoe during each walking activity (level-walking and stairs). The insoles were controlled by the iOS iPad app (loadsolT ${ }^{\mathrm{TM}}$, version 1.4.88, novel $\mathrm{GmbH}$, München, Germany). Before the start of the functional activities, the standard insole calibration procedure was performed after the participant donned the shoes and fastened the laces. Prior to testing in the DAO condition, the DAO was activated to reduce the loads under the braced foot by approximately $10 \%$ bodyweight as measured using the insoles in static double stance. A preferred walking speed was measured in $\mathrm{CON}$ before starting the series of functional activities in the first condition and after completing the series of functional activities in the second condition (i.e., pre- and post-test). This preferred walking speed was calculated from the measured time to complete two laps around two cones $25 \mathrm{ft}$ apart and used to determine if any experimental testing fatigue effects occurred. 
Table 5-1. Functional Activities

\begin{tabular}{ll}
\hline Functional Activity & \multicolumn{1}{c}{ Instructions for Each Activity } \\
\hline Standing & Hold double-legged static, quiet stance for 30 seconds. \\
Over-ground Walking & $\begin{array}{l}\text { Complete two laps around cones spaced } 25 \mathrm{ft} \text { apart at a self- } \\
\text { selected walking speed. }\end{array}$ \\
Treadmill Walking & $\begin{array}{l}\text { Walk on a treadmill for } 60 \text { seconds at a set speed (matching } \\
\text { the self-selected walking speed during over-ground walking). }\end{array}$ \\
& $\begin{array}{l}\text { If the handrail is used for balance, it should be used } \\
\text { consistently for both testing conditions. }\end{array}$ \\
& $\begin{array}{l}\text { Walk up } 20 \text { stairs. If the handrail is used for balance, it should } \\
\text { be used consistently for both testing conditions. }\end{array}$ \\
Stair Ascent & $\begin{array}{l}\text { Walk down } 20 \text { stairs. If the handrail is used for balance, it } \\
\text { should be used consistently for both testing conditions. If you } \\
\text { need to lead with a particular leg for stability, it should be } \\
\text { done consistently for both testing conditions. }\end{array}$ \\
Stair Descent & $\begin{array}{l}\text { Hold a single-legged static stance on the right side for } 30 \\
\text { seconds. If a hand on a wall is used for balance, it should be } \\
\text { used consistently for both testing conditions. }\end{array}$ \\
Single-leg Hold & $\begin{array}{l}\text { Hold a squat position for } 30 \text { seconds (as much bend at the } \\
\text { knees and ankles as possible while keeping the torso upright). } \\
\text { Note: The level of squat achieved varied between participants } \\
\text { due to differing levels of disability, but it was relatively the } \\
\text { same between testing conditions. }\end{array}$ \\
& \\
&
\end{tabular}


After completing the series of functional activities in both the unbraced and braced conditions to assess participant perception of DAO effectiveness, participants were administered a post-study questionnaire to gauge their experience in the DAO during testing. The post-study questionnaire included two questions: 1) "Did the brace (i.e., DAO) affect your overall symptoms during the activities performed?" and 2) "Did the brace (i.e., DAO) impact your ability to perform the activities?" The participant could answer from the following responses: "improved symptoms or function", "no change", or "worsened symptoms or function". The answer to the first question was used to classify participants into three groups based on the self-perceived symptoms due to acute DAO wear: "Improved Symptoms", "No Change", and "Worsened Symptoms." Participants were then asked to rate the magnitude of the change in function on a scale from -10 to +10 , with "+ 10" corresponding to "significantly improved function", 0 representing "no change in function", and "- 10" corresponding to "significantly worsened function." This value was then defined as participant Function Score. See Appendix E for extended methodology (Figures E-1 - E-4).

\section{Data Analyses}

The vertical force data were filtered using a Butterworth low-pass filter with a 50 $\mathrm{Hz}$ cutoff frequency using Visual3D software (C-Motion, Germantown, MD, USA) and used to compute right foot stance phase spatio-temporal and vertical force variables. A threshold of $20 \mathrm{~N}$ was used to define heel strike and toe-off. Stance time (for levelwalking data and stair data) was calculated from the force-time series as the time between heel strike and toe-off. For level-walking data, early stance was defined as the first half of stance phase (0-50\%), and late stance was defined as the second half of stance phase (50$100 \%$ ), and impact and push-off peak forces, instantaneous vertical loading rate, early stance impulse, and late stance impulse were computed. For the stair data, impulse and peak force were computed across the entire step (0-100\%).

\section{Statistical Analyses}

Shapiro-Wilk tests were used to test for normality of the preferred walking speed data and the vertical force data (SPSS, 25.0, IBM). For normally distributed data, paired t-tests were used to assess differences between conditions for each spatio-temporal and loading parameter. When the data were not normally distributed, Mann-Whitney U tests were used instead. Statistical significance was set a priori at $p<0.05$. Cohen's $d$ effect sizes were computed to assess mean difference magnitudes (small: $d<0.2$, moderate: 0.2 $\leq d \leq 0.8$; large: $d>0.8$ ). Pain Score data were treated as ordinal data and were analyzed using non-parametric Mann-Whitney $U$ tests to test for individual differences between conditions for each activity within symptoms groups. Eta-squared values $\left(\eta^{2}\right)$ were used to quantify the size of the effect for each difference (small: $\eta^{2} \leq 0.039$, moderate: $0.04<$ $\eta^{2}<0.139$, large: $\left.\eta^{2} \geq 0.14\right)$. 


\section{Results}

Patient demographics and characteristics are shown for all participants and for each symptom group in Table 5-2. According to the feedback questionnaire, the majority of participants reported that the brace improved their symptoms $(n=19)$, while a smaller group reported that the brace did not affect their symptoms one way or the other $(n=5)$. The remaining individual reported worsened symptoms with brace wear $(n=1)$. The function score was improved on average for all participants $(3.7 \pm 4.7)$, with greater improvement for the "Improved Symptoms" group ( $4.5 \pm 4.1)$, and still some improvement for the "No Change" group $(2.4 \pm 5.7)$. The "Worsened Symptoms" individual, however, had a negatively impacted function score of -6 . The distribution of diagnoses in each symptom group is described in Figure 5-2. Preferred walking speed during over-ground walking was not affected by the DAO (Table 5-3). Preferred walking speeds in the CON condition before and after testing were not different indicating that participants did not become fatigued by the activities performed.

The DAO reduced peak vertical forces during impact and push-off for both overground walking and level treadmill walking with small-to-moderate effect (Table 5-4). Loading rate was reduced with small effect size by the DAO during over-ground walking, but not during treadmill walking. Vertical impulse in early stance and late stance, and stance time were not affected by brace wear for either level-walking modality (Table 54). Peak vertical forces were reduced by the DAO during stair ascent and descent with small-to-moderate effect sizes (Table 5-4). Vertical impulse and stance time were not different between conditions during stair ascent and descent.

On average, the VAS responses showed that $46 \%$ of participants had improvement in perceived pain with DAO wear relative to CON, $40 \%$ had no changes, and 15\% had worsened pain (Table 5-5). For the "Improved Symptoms" group, 53\% had improvement in perceived pain on average with DAO wear relative to $\mathrm{CON}, 38 \%$ had no changes, and 9\% had worsened pain. For the "No Change" group, 25\% had improvement in pain on average, $53 \%$ had no changes, and $23 \%$ had worsened pain. For the more challenging functional activities (all excluding standing and sitting), the average DAO perceived pain was reduced relative to $\mathrm{CON}$ by 1.2 points, 1.6 points, and 0.1 points respectively for all participants, the "Improved Symptoms" group, and the "No Change" group. For the "Worsened Symptoms" individual, we observed an increase in average perceived pain with DAO by 1.3 points.

For all participants, the brace effect on perceived pain was not significant for any individual functional activity. However, the average perceived pain across all activities was significantly reduced by the DAO with large effect. The effect of the DAO on pain scores during treadmill walking and stair descent were approaching statistical significance. For the "Improved Symptoms" group, the DAO significantly improved the perceived pain of treadmill walking with moderate effect. The improvement in the perceived pain due to the DAO during stair ascent, stair descent, single-leg hold, and squat were approaching statistical significance with moderate effect. Again, the average perceived pain across all activities was significantly reduced by the DAO with large 
Table 5-2. Patient Demographics and Group Characteristics

\begin{tabular}{lcccc}
\hline Variable & $\begin{array}{c}\text { All } \\
\text { Participants } \\
(\mathbf{n}=\mathbf{2 5})\end{array}$ & $\begin{array}{c}\text { Improved } \\
\text { Symptoms } \\
(\mathbf{n}=\mathbf{1 9})\end{array}$ & $\begin{array}{c}\text { No Change in } \\
\text { Symptoms } \\
(\mathbf{n}=\mathbf{5})\end{array}$ & $\begin{array}{c}\text { Worsened } \\
\text { Symptoms } \\
(\mathbf{n}=\mathbf{1})\end{array}$ \\
\hline Men/Women & $8 / 17$ & $6 / 13$ & $2 / 3$ & $0 / 1$ \\
Age [years] & $42.6 \pm 14.1$ & $40.3 \pm 14.1$ & $54.6 \pm 5.9$ & 27 \\
Height $[\mathrm{cm}]$ & $170.1 \pm 8.6$ & $169.5 \pm 8.6$ & $172.2 \pm 10.4$ & 170.2 \\
Weight $[\mathrm{kg}]$ & $94.6 \pm 21.0$ & $98.5 \pm 20.4$ & $87.5 \pm 16.3$ & 56.2 \\
BMI $\left[\mathrm{kg} \cdot \mathrm{m}^{-2} \mathrm{~s}^{-2}\right]$ & $32.9 \pm 7.6$ & $34.4 \pm 7.3$ & $29.8 \pm 6.2$ & 19.4 \\
FADI & $55.6 \pm 18.2$ & $54.7 \pm 17.5$ & $62.3 \pm 22.3$ & 39.4 \\
Function Score & $3.7 \pm 4.7$ & $4.5 \pm 4.1$ & $2.4 \pm 5.7$ & -6 \\
\hline
\end{tabular}

Notes: Mean \pm SD; BMI: body mass index; FADI: Foot \& Ankle Disability Index. Function Score: the self-reported impact of the brace on the ability to complete tasks, reported on a scale from -10 to +10 , with "+ 10 " corresponding to "significantly improved function", 0 representing "no change in function", and "- 10" corresponding to "significantly worsened function." 
Improved Symptoms Group

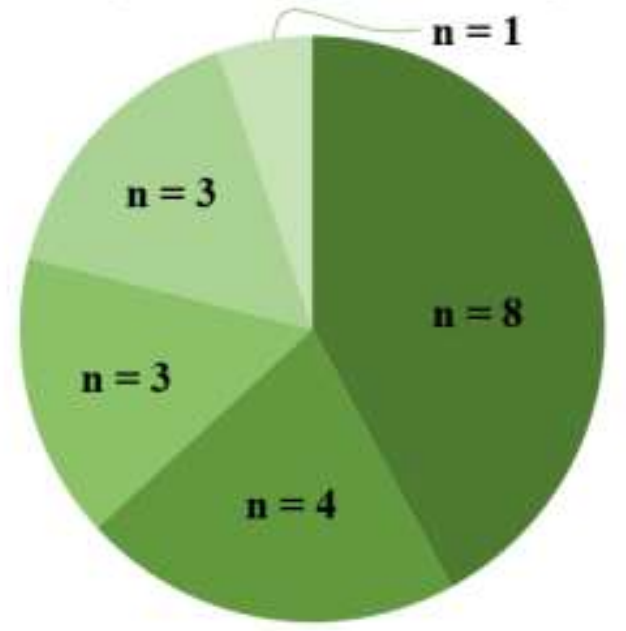

m Osteoarthritis

m Chronic Postoperative Pain

w Posterior Tibialis Tendon Dysfunction

Nerve Disorder

A) Severe Ankle Sprain
No Change in Symptoms Group

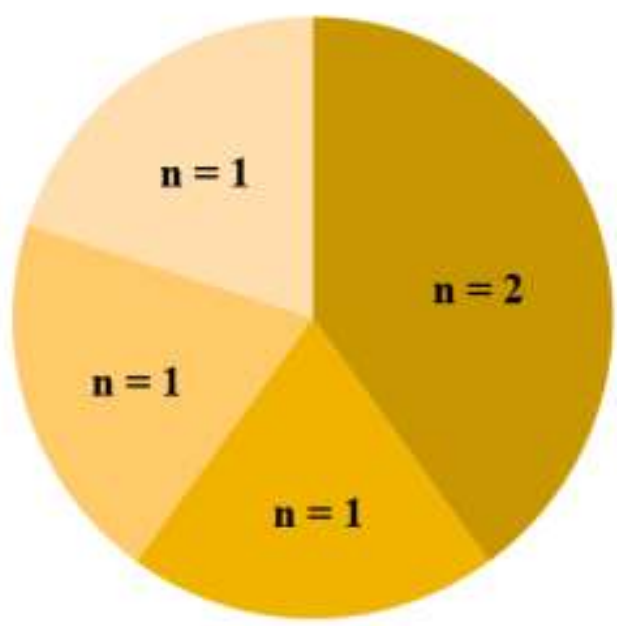

m Chronic Postoperative Pain

= Osteoarthritis

Nerve Disorder

Plantar Fasciitis

B)

Figure 5-2. Distribution of Diagnoses in the "Improved Symptoms" and "No Change in Symptoms" Groups

A) "Improved Symptoms" Group and B) "No Change in Symptoms" Group. The "Worsened Symptoms" group had only one participant, and that individual had a nerve disorder (tarsal tunnel). Individuals in the "Osteoarthritis" category had a diagnosis of osteoarthritis and, in some cases, a history of prior lower extremity fractures, midfoot osteotomy correction of Lisfranc deformity, pilon fracture, calcaneal stress fracture, syndesmotic injury, or osteochondral lesion. Individuals in the "Chronic Postoperative Pain" category included patients with histories of prior club foot procedure, medial malleolar osteotomy and osteochondral allograft, arthroscopically-assisted debridement and correction of micro-fracture of right medial osteochondral lesion, strayer procedure, closed reduction and medullary nailing of tibia, or cuboid fracture with the deltoid ligament disruption. Individuals in the "Nerve Disorder" category had diagnoses of tarsal tunnel, Morton's neuroma (3rd web space neuroma), or dorsal foot intermediate cutaneous neuritis. 
Table 5-3. Spatio-Temporal Variables of Walking Speed Before, During, and After Testing

\begin{tabular}{|c|c|c|c|c|}
\hline \multirow[b]{2}{*}{ Variable } & \multicolumn{2}{|c|}{$\begin{array}{c}\text { "Fatigue" Effects } \\
\text { (Standardized Footwear) }\end{array}$} & \multicolumn{2}{|c|}{ Over-ground Testing } \\
\hline & Before & After & CON & DAO \\
\hline $\begin{array}{l}\text { Preferred } \\
\text { Speed }\left[\mathrm{ms}^{-1}\right]\end{array}$ & $0.81 \pm 0.18$ & $0.84 \pm 0.24$ & $0.81 \pm 0.26$ & $0.76 \pm 0.24$ \\
\hline p-Value & \multicolumn{2}{|c|}{0.202} & \multicolumn{2}{|c|}{0.294} \\
\hline
\end{tabular}

Notes: Mean \pm SD; "Fatigue" effects were included to understand potential changes in preferred speed before and after the entire testing session to assess potential "fatigue" effects; CON: unbraced control condition; DAO: dynamic ankle orthosis condition. 
Table 5-4. Vertical In-Shoe Force and Spatio-Temporal Variables During Level-Walking and Stair Walking for Control and Dynamic Ankle-Foot Orthosis Conditions

\begin{tabular}{|c|c|c|c|c|c|c|}
\hline Activity & Loading Parameters & $\mathrm{CON}$ & DAO & p-Value & Effect $(d)$ & Interpretation \\
\hline \multirow[t]{6}{*}{ OGWALK } & Impact Peak Force [N] & $952.2 \pm 242.1$ & $887.7 \pm 228.5$ & $<0.001$ & 0.27 & Moderate \\
\hline & Push-off Peak Force [N] & $952.6 \pm 231.5$ & $902.3 \pm 219.0$ & $<0.001$ & 0.22 & Moderate \\
\hline & Early Stance Impulse $[\mathrm{N} \cdot \mathrm{s}]$ & $282.3 \pm 82.2$ & $274.2 \pm 85.7$ & 0.381 & 0.10 & Small \\
\hline & Late Stance Impulse $[\mathrm{N} \cdot \mathrm{s}]$ & $288.2 \pm 86.8$ & $276.5 \pm 83.4$ & 0.386 & 0.14 & Small \\
\hline & Loading Rate $\left[\mathrm{N} \cdot \mathrm{s}^{-1}\right]$ & $7080.1 \pm 3020.3$ & $6621.4 \pm 2909.3$ & 0.015 & 0.15 & Small \\
\hline & Stance Time $[\mathrm{s}]$ & $0.835 \pm 0.141$ & $0.859 \pm 0.163$ & 0.642 & 0.16 & Small \\
\hline \multirow[t]{6}{*}{ TMWALK } & Impact Peak Force [N] & $862.4 \pm 209.0$ & $803.2 \pm 187.3$ & 0.001 & 0.30 & Moderate \\
\hline & Push-off Peak Force [N] & $889.4 \pm 210.6$ & $860.0 \pm 201.5$ & 0.005 & 0.14 & Small \\
\hline & Early Stance Impulse $[\mathrm{N} \cdot \mathrm{s}]$ & $272.8 \pm 84.5$ & $258.2 \pm 73.6$ & 0.388 & 0.18 & Small \\
\hline & Late Stance Impulse $[\mathrm{N} \cdot \mathrm{s}]$ & $287.0 \pm 97.6$ & $271.4 \pm 79.4$ & 0.594 & 0.18 & Small \\
\hline & Loading Rate $\left[\mathrm{N} \cdot \mathrm{s}^{-1}\right]$ & $5779.0 \pm 2444.5$ & $5858.1 \pm 2467.5$ & 0.350 & 0.03 & Negligible \\
\hline & Stance Time $[\mathrm{s}]$ & $0.887 \pm 0.274$ & $0.882 \pm 0.242$ & 0.749 & 0.02 & Negligible \\
\hline \multirow[t]{3}{*}{ ASCENT } & Ascent Peak Force $[\mathrm{N}]$ & $918.5 \pm 221.7$ & $882.8 \pm 222.3$ & 0.001 & 0.16 & Small \\
\hline & Ascent Impulse $[\mathrm{N} \cdot \mathrm{s}]$ & $782.0 \pm 478.7$ & $762.6 \pm 518.8$ & 0.660 & 0.04 & Negligible \\
\hline & Ascent Stance Time $[\mathrm{s}]$ & $1.246 \pm 0.552$ & $1.259 \pm 0.606$ & 0.840 & 0.02 & Negligible \\
\hline \multirow[t]{3}{*}{ DESCENT } & Descent Peak Force $[\mathrm{N}]$ & $951.9 \pm 252.5$ & $900.6 \pm 205.7$ & 0.014 & 0.22 & Moderate \\
\hline & Descent Impulse [N·s] & $724.7 \pm 456.1$ & $696.1 \pm 309.1$ & 0.772 & 0.07 & Negligible \\
\hline & Descent Stance Time [s] & $1.129 \pm 0.563$ & $1.160 \pm 0.448$ & 0.481 & 0.06 & Negligible \\
\hline
\end{tabular}

Notes: $\mathrm{n}=25$; Mean $\pm \mathrm{SD}$; Bold $\mathrm{p}$-value: significant difference between conditions $(\mathrm{p}<0.05)$; Level-Walking: over-ground walking (OGWALK), treadmill walking (TMWALK), Early Stance Impulse: vertical impulse between 0 and 50\% of stance; Late Stance Impulse: vertical impulse between 50 and 100\% of stance; Loading Rate: loading rate of the peak impact force; Stair Walking: stair ascent (ASCENT), stair descent (DESCENT); Peak force: peak vertical force of entire step; Impulse: vertical impulse of entire step. 
Table 5-5. Distribution of Direction of VAS Pain Score Responses in DAO Relative to CON for Each Activity, and VAS Pain Scores for CON and DAO Conditions for All Participants, "Improved Symptoms," and "No Change in Symptoms"

\begin{tabular}{|c|c|c|c|c|c|c|c|c|c|}
\hline \multirow{2}{*}{ Group } & \multirow{2}{*}{ Activity } & \multicolumn{3}{|c|}{ Distribution of DAO Responses } & \multicolumn{5}{|c|}{ VAS Pain Scores (mean \pm SD) } \\
\hline & & Decrease & Same & Increase & CON & DAO & p-Value & Effect $\left(\eta^{2}\right)$ & Interpretation \\
\hline \multirow{9}{*}{ 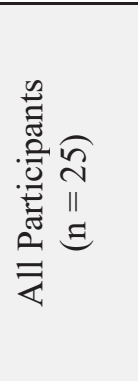 } & STAND & $32 \%$ & $40 \%$ & $28 \%$ & $3.1 \pm 2.8$ & $3.0 \pm 3.0$ & 0.813 & 0.001 & Negligible \\
\hline & OGWALK & $48 \%$ & $24 \%$ & $28 \%$ & $4.0 \pm 2.6$ & $3.4 \pm 2.9$ & 0.333 & 0.019 & Small \\
\hline & TMWALK & $48 \%$ & $40 \%$ & $12 \%$ & $4.0 \pm 2.5$ & $2.8 \pm 2.7$ & $0.084^{\mathrm{a}}$ & 0.061 & Moderate \\
\hline & ASCENT & $40 \%$ & $52 \%$ & $8 \%$ & $4.5 \pm 2.7$ & $3.2 \pm 2.8$ & 0.118 & 0.057 & Moderate \\
\hline & DESCENT & $52 \%$ & $44 \%$ & $4 \%$ & $4.5 \pm 2.6$ & $3.1 \pm 2.9$ & $0.078^{\mathrm{a}}$ & 0.072 & Moderate \\
\hline & SINGLE & $44 \%$ & $40 \%$ & $16 \%$ & $5.0 \pm 2.9$ & $3.8 \pm 3.2$ & 0.153 & 0.047 & Moderate \\
\hline & SQUAT & $56 \%$ & $32 \%$ & $12 \%$ & $5.5 \pm 3.2$ & $4.0 \pm 3.2$ & 0.128 & 0.054 & Moderate \\
\hline & SIT & $48 \%$ & $44 \%$ & $8 \%$ & $4.3 \pm 3.6$ & $3.2 \pm 3.3$ & 0.282 & 0.025 & Small \\
\hline & ALL & $46 \%$ & $40 \%$ & $15 \%$ & $4.3 \pm 0.7$ & $3.3 \pm 0.4$ & 0.005 & 0.483 & Large \\
\hline \multirow{9}{*}{ 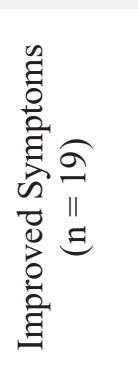 } & STAND & $37 \%$ & $37 \%$ & $26 \%$ & $3.5 \pm 2.9$ & $3.3 \pm 3.1$ & 0.708 & 0.004 & Negligible \\
\hline & OGWALK & $58 \%$ & $21 \%$ & $21 \%$ & $4.4 \pm 2.5$ & $3.5 \pm 2.9$ & 0.181 & 0.050 & Moderate \\
\hline & TMWALK & $58 \%$ & $42 \%$ & $0 \%$ & $4.5 \pm 2.4$ & $2.8 \pm 2.6$ & 0.027 & 0.133 & Moderate \\
\hline & ASCENT & $47 \%$ & $47 \%$ & $5 \%$ & $4.9 \pm 2.5$ & $3.3 \pm 2.8$ & $0.056^{\mathrm{a}}$ & 0.120 & Moderate \\
\hline & DESCENT & $53 \%$ & $47 \%$ & $0 \%$ & $5.0 \pm 2.3$ & $3.4 \pm 2.7$ & $0.056^{\mathrm{a}}$ & 0.119 & Moderate \\
\hline & SINGLE & $53 \%$ & $42 \%$ & $5 \%$ & $5.9 \pm 2.3$ & $4.1 \pm 3.0$ & $0.051^{\mathrm{a}}$ & 0.123 & Moderate \\
\hline & SQUAT & $63 \%$ & $32 \%$ & $5 \%$ & $6.1 \pm 2.9$ & $4.1 \pm 3.3$ & $0.080^{\mathrm{a}}$ & 0.102 & Moderate \\
\hline & SIT & $58 \%$ & $37 \%$ & $5 \%$ & $4.9 \pm 3.5$ & $3.4 \pm 3.4$ & 0.226 & 0.044 & Moderate \\
\hline & ALL & $53 \%$ & $38 \%$ & $9 \%$ & $4.9 \pm 0.8$ & $3.5 \pm 0.4$ & 0.001 & 0.645 & Large \\
\hline \multirow{9}{*}{ 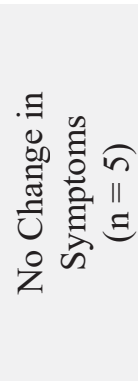 } & STAND & $20 \%$ & $60 \%$ & $20 \%$ & $1.6 \pm 2.6$ & $1.6 \pm 2.5$ & 0.841 & 0.006 & Negligible \\
\hline & OGWALK & $20 \%$ & $40 \%$ & $40 \%$ & $1.8 \pm 2.0$ & $2.2 \pm 2.7$ & 1.000 & 0.001 & Negligible \\
\hline & TMWALK & $20 \%$ & $40 \%$ & $40 \%$ & $1.8 \pm 2.0$ & $2.0 \pm 2.5$ & 1.000 & 0.001 & Negligible \\
\hline & ASCENT & $20 \%$ & $80 \%$ & $0 \%$ & $2.4 \pm 2.6$ & $2.2 \pm 2.3$ & 1.000 & 0.001 & Negligible \\
\hline & DESCENT & $60 \%$ & $40 \%$ & $0 \%$ & $2.2 \pm 2.5$ & $1.2 \pm 1.8$ & 0.548 & 0.050 & Moderate \\
\hline & SINGLE & $20 \%$ & $40 \%$ & $40 \%$ & $1.6 \pm 2.3$ & $1.8 \pm 2.5$ & 0.874 & 0.012 & Small \\
\hline & SQUAT & $40 \%$ & $40 \%$ & $20 \%$ & $2.8 \pm 3.1$ & $2.6 \pm 2.6$ & 1.000 & 0.001 & Negligible \\
\hline & SIT & $0 \%$ & $80 \%$ & $20 \%$ & $1.4 \pm 2.6$ & $1.6 \pm 2.6$ & 1.000 & 0.002 & Negligible \\
\hline & ALL & $25 \%$ & $53 \%$ & $23 \%$ & $2.0 \pm 0.5$ & $1.9 \pm 0.4$ & 0.959 & 0.001 & Negligible \\
\hline
\end{tabular}

Notes: Bold p-value: significant difference between conditions ( $\mathrm{p}<0.05)$; “a”: non-significant p-value approaching significance. 
effect. As expected, there were no significant improvements in perceived pain for any activity for the "No Change" group.

\section{Discussion}

This study was the initial clinical evaluation of a novel Dynamic Ankle Orthosis intended to provide pain relief and improve function for individuals suffering from mechanical foot and ankle pain. It was hypothesized that DAO wear would reduce underfoot mechanical loads and improve acute pain levels during the completion of functional activities compared to an unbraced control (CON). It was also hypothesized that participants would notice improvement in their overall pain symptoms and ability to do activities ("function") with DAO wear.

In agreement with our first hypothesis, we observed that the DAO reduced peak impact and push-off in-shoe forces during level over-ground walking and treadmill walking without affecting preferred walking speed or stance time. These findings are in agreement with our previous work showing that the DAO reduces peak plantar pressures during push-off in healthy individuals during level treadmill walking.[78] Further, the DAO reduced peak forces during stair ascent and descent compared to CON. Since the vertical forces applied below the foot were dampened despite the same mechanical demands during the functional activities (i.e., the only factor that was changed was brace condition), these findings suggest that the vertical forces bypassed anatomical structures and instead passed externally through the components of the brace. It is important to note however that we did not directly measure internal loads placed on the ankle joints or surrounding structures and therefore, it is difficult to conclude with certainty that mechanical loads on these structures were reduced.

Further, DAO wear reduced the loading rate of the vertical in-shoe force during over-ground walking, but not treadmill walking. This inconsistency is probably because the walking surface of the treadmill has inherent force-dampening effects due to its various flexible componentry. In over-ground walking, a reduction in loading rate means that the bodyweight shifted more slowly from the contralateral limb to the support limb with the DAO. The magnitude of the vertical loading rate has been shown to be consistent with axial joint loading rates of the lower extremity.[106] Higher axial joint loading rates have been shown to accelerate cartilage damage through the formation of surface fissures,[107] which can propagate mechanically through cyclic loading of the joint.[108] Therefore reduced loading rate with the DAO could slow the progression of joint damage in some degenerative clinical conditions such as OA/PTOA.

Contrary to our second hypothesis, we did not observe a consistent reduction in perceived pain levels with DAO wear during the functional activities. Furthermore, the noted reduction in peak vertical forces for level and stair walking did not correspond to a similar reduction in perceived pain except for treadmill walking in the "Improved Symptoms" group. Likely, a reduction in perceived pain was not observed for the overground walking activity because participants had not had adequate time to adjust or 
acclimate to walking in the brace. However, a significant reduction in perceived pain with the DAO became evident when pain levels were averaged across the series of activities for all participants and for the "Improved Symptoms" group. This finding suggests that the DAO had an overall positive effect on pain levels during the series of functional tasks even though a brace effect was not consistently seen during the individual tasks.

The average DAO perceived pain for the more challenging functional activities (all tasks excluding standing and sitting) was reduced relative to CON by 1.2 points, 1.6 points, and 0.1 points for all participants, the "Improved Symptoms" group, and the "No Change" group, respectively. Since the measurement of pain is highly subjective, there are conflicting reports on which level of change in perceived pain indicates a clinically "meaningful" change or Minimum Clinically Important Difference (MCID). When selecting a reference MCID, it is important to consider factors such as the cause of the pain, timing and duration of the pain, region of the body affected, and treatment type. A broad range of values (0.9 - 1.3 points) is used as the MCID for general pain scores with a VAS.[109,110] There is conflicting evidence regarding whether or not the MCID is uniform along the entire VAS.[111,112] For foot and ankle issues, MCID values range from 0.8 points for conservative treatments to 1.8 points for surgical treatments.[113-115] Therefore, since the present study evaluated the effect of a conservative treatment option for the foot and ankle, it can be reasonably concluded that the 1.2 to 1.6 point reduction in pain score with the DAO represents a clinically meaningful improvement in pain.

Each individual in the present study was functionally impaired to some degree by their condition ("moderately disabled" per average FADI score of 55.6 $\pm 18.2 \%$, where $100 \%$ represents no disability).[97] With DAO wear, as hypothesized, most participants reported improvement in their overall symptoms and function (from questionnaire response). The diagnoses that benefitted the most from the DAO were OA/PTOA, posterior tibialis tendon dysfunction (PTTD), chronic postoperative pain (CPOP), severe ankle sprain, and nerve disorders. Although each of these diseases has a different trigger for pain, they are all caused primarily by mechanical factors. OA typically results from damage to the articular cartilage within the joint, which then causes bone-on-bone rubbing to occur during ambulation. $[8,10]$ In cases of PTTD, the posterior tibialis tendon is inflamed, stretched, or torn, which causes the ankle to be misaligned and the arch of the foot to flatten.[116] Severe ankle sprains occur when a ligament is completely torn, and they can result in permanent instability of the joint and extreme pain during weightbearing.[29-32] CPOP often presents following orthopedic procedures as persistent movement-evoked pain that lasts months or years after the surgical site is healed.[37-40] Nerve disorders typically involve damaged or thickened nerve tissue on the plantar aspect of the foot resulting from irritation of the nerve by mechanical factors such as compression.[41] In cases of OA/PTOA, PTTD, and ankle sprains, we hypothesize that the DAO provided a reduction in natural joint compressive forces and improved the overall joint alignment as the primary mechanisms of pain relief and functional improvement. The reduction in peak loading parameters seen here and in prior studies likely corresponded to some reduction in intraarticular joint forces.[77,78] Although the effect of the DAO on joint alignment was not measured in the present study, the DAO 
has been shown previously to provide controlled, natural sagittal and frontal plane ankle kinematics. [77,78] In cases of CPOP and nerve disorders we hypothesize that, in addition to the reduced loads, the reinforced components of the DAO provided helpful stability to portions of the lower leg and foot where free joint motion normally provoked pain as a secondary mechanism of pain relief and functional improvement. Overall, these findings suggest that a link between pain levels and function may exist and warrants further investigation within specific clinical populations.

As with all studies, there are certain limitations that must be considered when interpreting these findings. The individuals were recruited for this study based on their symptoms more so than based on their specific diagnosis. Since this was the case, there were a variety of diagnoses included in the study and this type of intervention may not have been appropriate for all participants. Future work should narrow the inclusion criteria to a specific diagnosis to eliminate variation caused by different pain mechanisms. The DAO was activated to about $10 \%$ bodyweight in this study, but past work has shown that the DAO can offload up to $30 \%$ bodyweight comfortably. Since there is a known dose-effect for weight loss on pain relief,[48] future work should consider investigating the relationship between DAO offloading levels and pain relief to see if a similar dose-effect exists. For the stair walking, single-leg hold, and squat activities, participants were given the freedom to complete these tasks with certain modifications if necessary, so long as it was repeated in both conditions. As such, some individuals used the handrail and some did not, and some individuals used a hand on the wall for stability while others did not. However, the effect of these modifications was minimized by requiring them to be done similarly in both conditions.

\section{Conclusion}

In this study, the acute benefits of DAO wear were evaluated during various functional activities in patients with a broad range of symptomatic foot and ankle diseases. Through the evaluation of in-shoe forces, pain levels, and reported symptoms, this study has provided novel evidence that the DAO can effectively improve symptoms for patients with OA/PTOA, PTTD, CPOP, ankle sprains, and nerve disorders. Future work is warranted to investigate the effects of extended or long-term DAO wear in a specific clinical population such as OA/PTOA, PTTD, or CPOP. 


\section{CHAPTER 6. FUTURE DIRECTIONS}

Chapter 6 describes the path to commercialization that has been traversed so far for the DAO as well as some considerations for future research and concluding remarks.

\section{Path to Commercialization}

The path to medical device commercialization is a long and tedious one. Over the course of years, an idea can turn into a marketable product by working through the stages of product development. Every idea begins in the concept and design phase, during which the clinical problem is identified through some form of needs assessment. A proposed solution (i.e., the device) is conceptualized with this unmet need in mind and similar products are identified through a competitive assessment. At this stage, it is important to identify the customer or who would be using the device in the end by performing a market analysis and customer interviews. Obtaining customer input early on is important for the refinement of the design as well as for the identification of the risks of the device to the end user. Finally, no idea can move forward without the proper support framework in place, so funds must be secured through stakeholders, grants, or other means. To convince investors to support the concept, a business plan and payment strategy must be developed to understand how profits will be made from the device such as through a reimbursement model.

For the DAO, we identified that there is a specific type of pain of the foot and ankle that is triggered by mechanical means, specifically weight-bearing and movement of the joint. We hypothesized that this type of pain could be alleviated by reducing the loads passing through the joint. The proposed solution was to provide offloading through an external device (i.e., orthosis) that spanned across the foot and ankle without interfering with joint motion. Through conversations with local orthotists, orthopedic surgeons, and physical therapists (i.e., the customers), we determined that there was a need for this device in populations with mechanical foot and ankle pain, specifically patients with osteoarthritis (i.e., the end user), and that there weren't any other similar devices on the market. Funding was secured through internal grants to support further development of the device. While establishing partnerships with the main customer candidates for this device, orthotic fabrication companies and local orthotists, it was determined that the use of existing medical billing codes (i.e., L1990, L1960, L1906) would provide reimbursement for the services rendered when prescribing and fabricating the DAO.

The next phase along the path to commercialization of a medical device involves transforming the concept into a feasible prototype. This stage of development is an iterative process of prototyping, validation, verification, and reengineering. Keeping track of the design history during this phase is essential for navigating through the complex regulatory pathway that awaits. To bring a new medical device to market in the United States, manufacturers must meet the Food and Drug Administration (FDA) requirements 
to establish that the medical device is safe and effective. New medical devices are subjected to different levels of regulatory control based on their classification. There are three medical device classifications defined by the risk posed to the user and the intended usage of the device, with Class I being lowest risk and Class III being highest risk.

The DAO would be considered a Class I Exempt device (21 CFR Part 890 Subpart D Subpart 890.3475 Limb Orthosis), and therefore a premarket notification application and FDA clearance is not required before marketing the device in the U.S. However, registration of the manufacturer's establishment and a listing for the generic category or classification name is required. To raise the bar for entry of potential competitors down the road, a higher classification could be pursued. In addition to the registration requirements, the FDA requires manufacturers of Class I Exempt devices to adhere to Quality System Regulation and Good Manufacturing Practices when the device reaches the manufacturing stage.

Finally, once a new device has been well conceptualized, the inventors should endeavor to protect their intellectual property by filing a patent application. Prior to beginning the benchtop validation work described in Chapter 3, there was a significant amount of time invested in developing and improving the device design for optimal comfort and efficacy. This iterative process resulted in several prototype variants that eventually went on to be included in the patent application for the invention. Through the University of Tennessee Research Foundation, the patent application was submitted: United States Patent Application Serial No. 62/481,741 and PCT International Application Serial No. PCT/US2018/026248 "DYNAMIC ANKLE ORTHOSIS DEVICES, SYSTEMS, AND METHODS” (Figure 6-1).[76]

Although the FDA does not require evidence of product safety and efficacy for low risk, Class I exempt devices, scientific research and trials are still necessary to support the marketing claims of the device and to convince insurance companies to provide coverage for the device. The benchtop validation work described in Chapter 3 was performed to validate the design goals of the DAO (i.e., offloading capacity and joint mobility). The functional assessment described in Chapter 4 provided further validation of the design goals in a functional setting, during walking with healthy individuals. Finally, the clinical assessment described in Chapter 5 provided convincing evidence of the efficacy of the device to achieve the end goal of reducing pain and improving function in patients with painful impairments of the foot and ankle.

\section{Considerations for Future Research}

The work described in the previous chapters set the foundation for further development of the DAO. However, there are several points to consider in future work with this device. Chapter 3 describes the basic construction of the device. Throughout the development of the device, there have been several iterations and improvements of the design, each less complex than the previous. For wearable medical devices, I've learned 

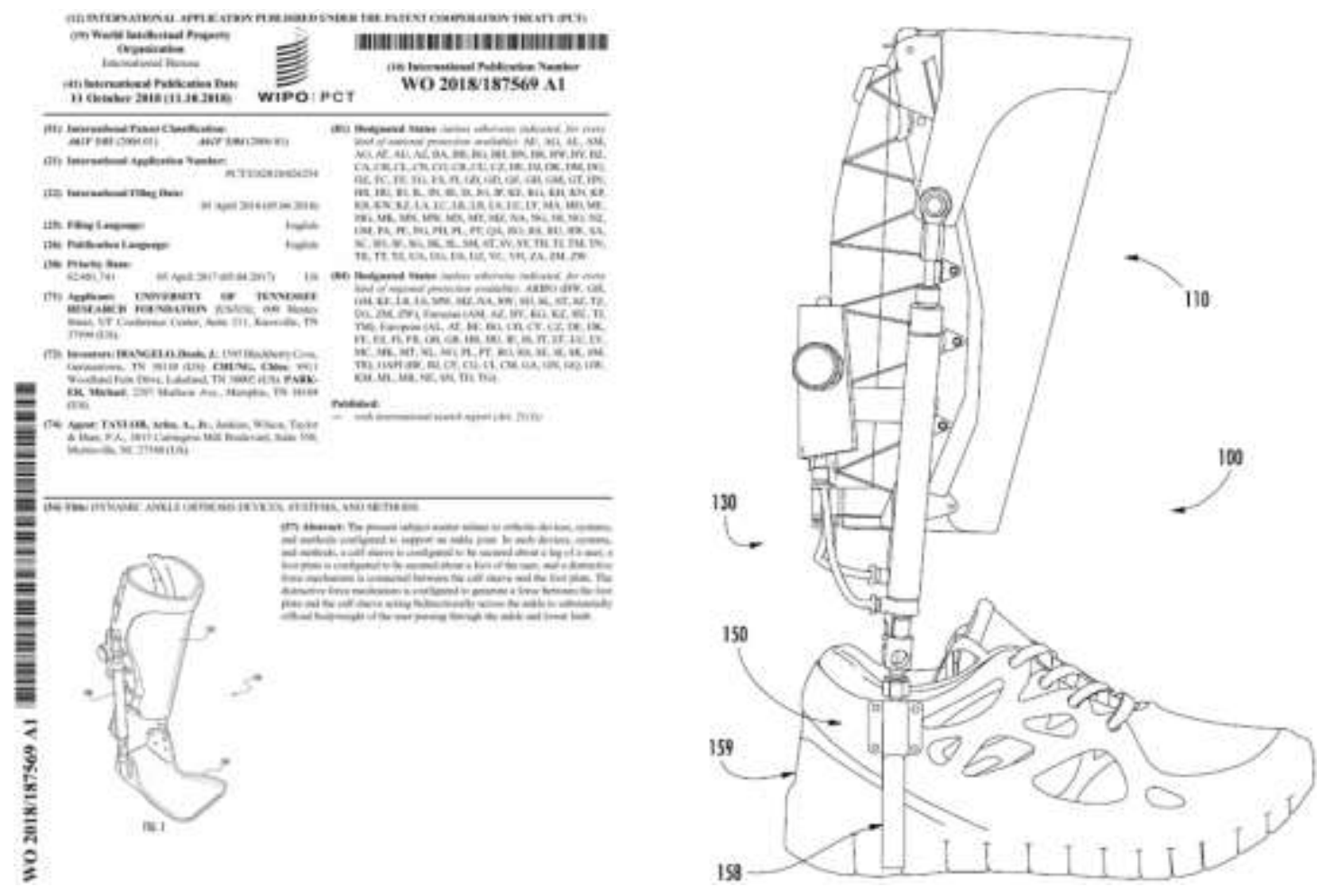

Figure 6-1. Patent Application for the Dynamic Ankle Orthosis 
that it is crucial to 1) minimize fabrication costs, 2) maximize comfort, and 3) simplify operation. In Chapters 4 and 5, pressure insoles and force insoles were used to capture underfoot loading with the brace. These pieces of equipment extended the study of the device beyond the laboratory setting, but they also were limited in that they could only provide measures of loads normal to the plantar aspect of the foot. This trade-off was necessary for the present work, but future work should consider utilizing more sophisticated devices to measure other biomechanical parameters such as the anteroposterior ground reaction forces (i.e., braking and propulsive forces), force attenuation, and center of pressure. Another consideration for future work is to investigate the potential dose-effect between DAO offloading and pain relief. In the present work, the DAO was activated to about $10 \%$ bodyweight, but there is evidence that there exists a dose-effect for weight loss on pain relief. If a similar dose-effect exists for foot and ankle pain and offloading, this relationship is something that should be investigated and considered in the prescription of the device.

The clinical assessment of the DAO described in Chapter 5 provided evidence that the DAO can effectively improve symptoms for patients with OA/PTOA, PTTD, CPOP, ankle sprains, and nerve disorders. Each of these diagnoses is different in terms of their mechanism for pain. Therefore, future work is warranted to investigate the effects of extended or long-term DAO wear in a specific clinical population such as OA/PTOA, PTTD, or CPOP to better understand how exactly the DAO alleviates symptoms of each condition. Inclusion of periodic radiographic imaging and measurements of ankle joint alignment with and without the brace would be helpful in understanding the mechanism for chronic relief of symptoms. To better understand the effect of the DAO on day-to-day function, future work should include measurements of daily activity levels, time spent wearing the brace, and daily pain scores. Ultimately, the DAO could serve as an at-home aid, as a therapeutic rehabilitation tool for use within the clinic, or in another context altogether. To support the wide implementation of the DAO, suitable healthcare modalities need to be explored and defined. Finally, the use of the DAO may extend beyond clinical and healthcare settings. The effects of the DAO on injury prevention, injury treatment, and performance need to be explored in other populations such as athletes and the military.

\section{Conclusion}

This body of work demonstrates the capacity of the DAO to provide significant offloading during ambulation without greatly affecting kinematic parameters including sagittal and frontal plane ankle motion compared to an unbraced control. Additionally,

this work provides novel evidence that the DAO can improve symptoms and the ability of impaired individuals to complete functional activities such as level and stair walking. 


\section{LIST OF REFERENCES}

1. Parker-Pope, T. (2010, Jan. 18, 2010). A New Joint Gains as a Candidate for Replacement. The New York Times. Retrieved from http://www.nytimes.com/2010/01/19/health/19well.html on December 5, 2016.

2. Gates, L. S., Arden, N. K., Hannan, M. T., Roddy, E., Gill, T. K., Hill, C. L., \& Golightly, Y. M. (2019). Prevalence of Foot Pain Across an International Consortium of Population-Based Cohorts. Arthritis Care Res (Hoboken), 71(5), 661-670. https://doi.org/10.1002/acr.23829

3. Peat, G., Thomas, E., Wilkie, R., \& Croft, P. (2006). Multiple joint pain and lower extremity disability in middle and old age. Disabil Rehabil, 28(24), 15431549. https://doi.org/10.1080/09638280600646250

4. Menz, H. B., Morris, M. E., \& Lord, S. R. (2005). Foot and ankle characteristics associated with impaired balance and functional ability in older people. The Journals of Gerontology Series A: Biological Sciences and Medical Sciences, 60(12), 1546-1552. https://doi.org/10.1093/gerona/60.12.1546

5. Menz, H. B., Morris, M. E., \& Lord, S. R. (2006). Foot and ankle risk factors for falls in older people: a prospective study. The Journals of Gerontology Series A: Biological Sciences and Medical Sciences, 61(8), 866-870. https://doi.org/10.1093/gerona/61.8.866

6. $\quad$ Mickle, K. J., Munro, B. J., Lord, S. R., Menz, H. B., \& Steele, J. R. (2011). Cross-sectional analysis of foot function, functional ability, and health-related quality of life in older people with disabling foot pain. Arthritis Care Res (Hoboken), 63(11), 1592-1598. https://doi.org/10.1002/acr.20578

7. Hill, C. L., Gill, T. K., Menz, H. B., \& Taylor, A. W. (2008). Prevalence and correlates of foot pain in a population-based study: the North West Adelaide health study. J Foot Ankle Res, 1(1), 2. https://doi.org/10.1186/1757-1146-1-2

8. Barg, A., Pagenstert, G. I., Hugle, T., Gloyer, M., Wiewiorski, M., Henninger, H. B., \& Valderrabano, V. (2013). Ankle osteoarthritis: etiology, diagnostics, and classification. Foot Ankle Clin, 18(3), 411-426. https://doi.org/10.1016/j.fcl.2013.06.001

9. Nguyen, M. P., Pedersen, D. R., Gao, Y., Saltzman, C. L., \& Amendola, A. (2015). Intermediate-term follow-up after ankle distraction for treatment of endstage osteoarthritis. J Bone Joint Surg Am, 97(7), 590-596.

https://doi.org/10.2106/jbjs.n.00901

10. Saltzman, C. L., Zimmerman, M. B., O'Rourke, M., Brown, T. D., Buckwalter, J. A., \& Johnston, R. (2006). Impact of comorbidities on the measurement of health in patients with ankle osteoarthritis. J Bone Joint Surg Am, 88(11), 23662372. https://doi.org/10.2106/jbjs.F.00295

11. Hunter, D. J., \& Lo, G. H. (2009). The management of osteoarthritis: an overview and call to appropriate conservative treatment. Med Clin North Am, 93(1), 127-143, xi. https://doi.org/10.1016/j.mcna.2008.07.009

12. DeMik, D. E., Bedard, N. A., Dowdle, S. B., Burnett, R. A., McHugh, M. A., \& Callaghan, J. J. (2017). Are We Still Prescribing Opioids for Osteoarthritis? J 
Arthroplasty, 32(12), 3578-3582.e3571.

https://doi.org/10.1016/j.arth.2017.07.030

13. Brockett, C. L., \& Chapman, G. J. (2016). Biomechanics of the ankle.

Orthopaedics and Trauma, 30(3), 232-238.

https://doi.org/10.1016/j.mporth.2016.04.015

14. Ewalefo, S. O., Dombrowski, M., Hirase, T., Rocha, J. L., Weaver, M., Kline, A., Carney, D., \& Hogan, M. V. (2018). Management of Posttraumatic Ankle Arthritis: Literature Review. Curr Rev Musculoskelet Med, 11(4), 546-557. https://doi.org/10.1007/s12178-018-9525-9

15. Woodburn, J., Barker, S., \& Helliwell, P. S. (2002). A randomized controlled trial of foot orthoses in rheumatoid arthritis. J Rheumatol, 29(7), 1377-1383. https://www.ncbi.nlm.nih.gov/pubmed/12136891

16. Thompson, J. A., Jennings, M. B., \& Hodge, W. (1992). Orthotic therapy in the management of osteoarthritis. J Am Podiatr Med Assoc, 82(3), 136-139. https://doi.org/10.7547/87507315-82-3-136

17. Kitaoka, H. B., Crevoisier, X. M., Harbst, K., Hansen, D., Kotajarvi, B., \& Kaufman, K. (2006). The effect of custom-made braces for the ankle and hindfoot on ankle and foot kinematics and ground reaction forces. Arch Phys Med Rehabil, 87(1), 130-135. https://doi.org/10.1016/j.apmr.2005.08.120

18. Radtka, S. A., Oliveira, G. B., Lindstrom, K. E., \& Borders, M. D. (2006). The kinematic and kinetic effects of solid, hinged, and no ankle-foot orthoses on stair locomotion in healthy adults. Gait Posture, 24(2), 211-218. https://doi.org/10.1016/j.gaitpost.2005.09.005

19. Betts, J. G., Johnson, E., Wise, J. A., \& Young, K. A. (2020). Bones of the Lower Limb. In Anatomy and Physiology: OpenStax. Retrieved from https://opentextbc.ca/anatomyandphysiology/chapter/8-4-bones-of-the-lower$\underline{\mathrm{imb} /}$ on January 16, 2020.

20. Neumann, D. (2010). "Ankle and Foot" and "Kinesiology of Walking”. In Kinesiology of the Musculoskeletal System: Foundations of Physical Rehabilitation (2nd ed., pp. 574-580, pp.636): Mosby. ISBN-13: 9780323039895

21. Stauffer, R. N., Chao, E. Y. S., \& Brewster, R. C. (1977). Force and Motion Analysis of the Normal, Diseased, and Prosthetic Ankle Joint. Clinical Orthopaedics and Related Research $₫(127), 189-196$.

https://www.ncbi.nlm.nih.gov/pubmed/912978

22. Castro, M. D. (2002). Ankle biomechanics. Foot and Ankle Clin N Am, 7(4), 679-693. https://doi.org/10.1016/s1083-7515(02)00049-9

23. Sanford, B., Williams, J., Zucker-Levin, A., \& Mihalko, W. (2014). Hip, Knee, and Ankle Joint Forces in Healthy Weight, Overweight, and Obese Individuals During Walking. In: Computational Biomechanics for Medicine (pp. 101-111). Doyle B., Miller K., Wittek A., Nielsen P. (eds). Springer, New York, NY. https://doi.org/10.1007/978-1-4939-0745-8

24. Hills, A. P., Hennig, E. M., McDonald, M., \& Bar-Or, O. (2001). Plantar pressure differences between obese and non-obese adults: a biomechanical analysis. International Journal of Obesity, 25(11), 1674-1679. https://doi.org/10.1038/sj.ijo.0801785 
25. Perry, J. \& Burnfield, J. M. (2010). Gait Analysis: Normal and Pathological Function. Journal of sports science \& medicine, 9(2), 353-353. ISBN-13: 9781556427664

26. Kuo, A. D., \& Donelan, J. M. (2010). Dynamic principles of gait and their clinical implications. Phys Ther, 90(2), 157-174.

https://doi.org/10.2522/ptj.20090125

27. Malanga, G., \& DeLisa, J. (1998). Clinical observation. Gait analysis in the science of rehabilitation, 1-10. Retrieved from https://www.rehab.research.va.gov/mono/gait/malanga.pdf on October 18, 2019.

28. Lambers, K., Ootes, D., \& Ring, D. (2012). Incidence of patients with lower extremity injuries presenting to US emergency departments by anatomic region, disease category, and age. Clin Orthop Relat Res, 470(1), 284-290.

https://doi.org/10.1007/s11999-011-1982-z

29. Doherty, C., Delahunt, E., Caulfield, B., Hertel, J., Ryan, J., \& Bleakley, C. (2014). The incidence and prevalence of ankle sprain injury: a systematic review and meta-analysis of prospective epidemiological studies. Sports Med, 44(1), 123-140. https://doi.org/10.1007/s40279-013-0102-5

30. Mauntel, T. C., Wikstrom, E. A., Roos, K. G., Djoko, A., Dompier, T. P., \& Kerr, Z. Y. (2017). The Epidemiology of High Ankle Sprains in National Collegiate Athletic Association Sports. Am J Sports Med, 45(9), 2156-2163. https://doi.org/10.1177/0363546517701428

31. Waterman, B. R., Owens, B. D., Davey, S., Zacchilli, M. A., \& Belmont, P. J., Jr. (2010). The epidemiology of ankle sprains in the United States. J Bone Joint Surg Am, 92(13), 2279-2284. https://doi.org/10.2106/jbjs.I.01537

32. Fong, D. T., Hong, Y., Chan, L. K., Yung, P. S., \& Chan, K. M. (2007). A systematic review on ankle injury and ankle sprain in sports. Sports Med, 37(1), 73-94. https://doi.org/10.2165/00007256-200737010-00006

33. U.S. Department of Labor Bureau of Labor Statistics. News Release: Nonfatal Occupational Injuries and Illnesses Requiring Days Away from Work, 2014. November 19, 2015. Retrieved from https://www.bls.gov/news.release/pdf/osh2.pdf on December 5, 2016.

34. Washington State Department of Labor \& Industries L\&I Workers' Compensation Claims 2007-2016 Number of Claims by Body Part Injured. Retrieved from https://www.lni.wa.gov/claims/for-employers/workerscompensation-injury-data/injury-data on December 5, 2016.

35. Thomas, M. J., Roddy, E., Zhang, W., Menz, H. B., Hannan, M. T., \& Peat, G. M. (2011). The population prevalence of foot and ankle pain in middle and old age: a systematic review. Pain, 152(12), 2870-2880.

https://doi.org/10.1016/j.pain.2011.09.019

36. Dunn, J. E., Link, C. L., Felson, D. T., Crincoli, M. G., Keysor, J. J., \& McKinlay, J. B. (2004). Prevalence of foot and ankle conditions in a multiethnic community sample of older adults. Am J Epidemiol, 159(5), 491-498. https://doi.org/10.1093/aje/kwh071

37. Hoofwijk, D. M., Fiddelers, A. A., Emans, P. J., Joosten, E. A., Gramke, H. F., Marcus, M. A., \& Buhre, W. F. (2015). Prevalence and Predictive Factors of Chronic Postsurgical Pain and Global Surgical Recovery 1 Year After 
Outpatient Knee Arthroscopy: A Prospective Cohort Study. Medicine (Baltimore), 94(45), e2017. https://doi.org/10.1097/md.0000000000002017

38. Correll, D. (2017). Chronic postoperative pain: recent findings in understanding and management. F1000Res, 6, 1054.

https://doi.org/10.12688/f1000research.11101.1

39. Bjørnholdt, K. T., Brandsborg, B., Søballe, K., \& Nikolajsen, L. (2015).

Persistent pain is common 1-2 years after shoulder replacement. Acta orthopaedica, 86(1), 71-77. https://doi.org/10.3109/17453674.2014.987065

40. Pogatzki-Zahn, E. M., Segelcke, D., \& Schug, S. A. (2017). Postoperative painfrom mechanisms to treatment. Pain reports, 2(2), e588-e588. https://doi.org/10.1097/PR9.0000000000000588

41. Park, C. H., \& Chang, M. C. (2019). Forefoot disorders and conservative treatment. Yeungnam Univ J Med, 36(2), 92-98. https://doi.org/10.12701/yujm.2019.00185

42. Landro, L. (2014). The Informed Patient: More Patients Opt to Replace Ankle Joints Instead of Fusion. The Wall Street Journal. Retrieved from http://www.wsj.com/articles/more-patients-opt-to-replace-ankle-joints-instead-o f-fusion-1416867103 on December 5, 2016.

43. Belatti, D. A., \& Phisitkul, P. (2014). Economic burden of foot and ankle surgery in the US Medicare population. Foot Ankle Int, 35(4), 334-340. https://doi.org/10.1177/1071100713519777

44. Janisse, D. J., \& Janisse, E. (2008). Shoe Modification and the Use of Orthoses in the Treatment of Foot and Ankle Pathology. JAAOS - Journal of the American Academy of Orthopaedic Surgeons, 16(3), 152-158. https://doi.org/10.5435/00124635-200803000-00006

45. Kobayashi, T., Leung, A. K. L., \& Hutchins, S. W. (2011). Techniques to measure rigidity of ankle-foot orthosis: A review. The Journal of Rehabilitation Research and Development, 48(5). https://doi.org/10.1682/jrrd.2010.10.0193

46. Elattar, O., Smith, T., Ferguson, A., Farber, D., \& Wapner, K. (2018). Uses of Braces and Orthotics for Conservative Management of Foot and Ankle Disorders. Foot \& Ankle Orthopaedics, 3(3), 2473011418780700. https://doi.org/10.1177/2473011418780700

47. National Institutes of Health (1998). Clinical guidelines on the identification, evaluation, and treatment of overweight and obesity in adults: executive summary. Expert Panel on the Identification, Evaluation, and Treatment of Overweight in Adults. Am J Clin Nutr, 68(4), 899-917. https://doi.org/10.1093/ajcn/68.4.899

48. Gersing, A. S., Solka, M., Joseph, G. B., Schwaiger, B. J., Heilmeier, U., Feuerriegel, G., Nevitt, C., McCulloch, C. E., \& Link, T. M. (2016).

Progression of cartilage degeneration and clinical symptoms in obese and overweight individuals is dependent on the amount of weight loss: 48-month data from the Osteoarthritis Initiative. Osteoarthritis Cartilage, 24(7), 11261134. https://doi.org/10.1016/j.joca.2016.01.984

49. Atukorala, I., Makovey, J., Lawler, L., Messier, S. P., Bennell, K., \& Hunter, D. J. (2016). Is There a Dose-Response Relationship Between Weight Loss and 
Symptom Improvement in Persons With Knee Osteoarthritis? Arthritis Care Res (Hoboken), 68(8), 1106-1114. https://doi.org/10.1002/acr.22805

50. Messier, S. P., Loeser, R. F., Miller, G. D., Morgan, T. M., Rejeski, W. J., Sevick, M. A., Ettinger Jr., W. H., Pahor, M., \& Williamson, J. D. (2004).

Exercise and dietary weight loss in overweight and obese older adults with knee osteoarthritis: the Arthritis, Diet, and Activity Promotion Trial. Arthritis Rheum, 50(5), 1501-1510. https://doi.org/10.1002/art.20256

51. Messier, S. P., Mihalko, S. L., Legault, C., Miller, G. D., Nicklas, B. J., DeVita, P., Beavers, D. P., Hunter, D. J., Lyles, M. F., Eckstein, F., Williamson, J. D., Carr, J. J., Guermazi, A., \& Loeser, R. F. (2013). Effects of intensive diet and exercise on knee joint loads, inflammation, and clinical outcomes among overweight and obese adults with knee osteoarthritis: the IDEA randomized clinical trial. Jama, 310(12), 1263-1273. https://doi.org/10.1001/jama.2013.277669

52. Messier, S. P., Resnik, A. E., Beavers, D. P., Mihalko, S. L., Miller, G. D., Nicklas, B. J., DeVita, P., Hunter, D. J., Lyles, M. F., Eckstein, F., Guermazi, A., \& Loeser, R. F. (2018). Intentional Weight Loss in Overweight and Obese Patients With Knee Osteoarthritis: Is More Better? Arthritis Care Res (Hoboken), 70(11), 1569-1575. https://doi.org/10.1002/acr.23608

53. Alcalde, G. E., Fonseca, A. C., Boscoa, T. F., Goncalves, M. R., Bernardo, G. C., Pianna, B., Carnavale, B. F., Gimenes, C., Barrile, S. R., \& Arca, E. A. (2017). Effect of aquatic physical therapy on pain perception, functional capacity and quality of life in older people with knee osteoarthritis: study protocol for a randomized controlled trial. Trials, 18(1), 317. https://doi.org/10.1186/s13063-017-2061-x

54. Dias, J. M., Cisneros, L., Dias, R., Fritsch, C., Gomes, W., Pereira, L., Santos, M. L., \& Ferreira, P. H. (2017). Hydrotherapy improves pain and function in older women with knee osteoarthritis: a randomized controlled trial. Braz J Phys Ther, 21(6), 449-456. https://doi.org/10.1016/j.bjpt.2017.06.012

55. Mehrholz, J., Thomas, S., \& Elsner, B. (2017). Treadmill training and body weight support for walking after stroke. Cochrane Database Syst Rev, 8, Cd002840. https://doi.org/10.1002/14651858.CD002840.pub4

56. Perez de la Cruz, S. (2017). Effectiveness of aquatic therapy for the control of pain and increased functionality in people with Parkinson's disease: a randomized clinical trial. Eur J Phys Rehabil Med, 53(6), 825-832. https://doi.org/10.23736/s1973-9087.17.04647-0

57. Perez Ramirez, N., Nahuelhual Cares, P., \& San Martin Penailillo, P. (2019). Effectiveness of Watsu therapy in patients with juvenile idiopathic arthritis. A parallel, randomized, controlled and single-blind clinical trial. Rev Chil Pediatr, 90(3), 283-292. https://doi.org/10.32641/rchped.v90i3.886

58. Patil, S., Steklov, N., Bugbee, W. D., Goldberg, T., Colwell, C. W., Jr., \& D'Lima, D. D. (2013). Anti-gravity treadmills are effective in reducing knee forces. J Orthop Res, 31(5), 672-679. https://doi.org/10.1002/jor.22272

59. Hjartarson, H. F., \& Toksvig-Larsen, S. (2018). The clinical effect of an unloader brace on patients with osteoarthritis of the knee, a randomized placebo 
controlled trial with one year follow up. BMC Musculoskelet Disord, 19(1), 341. https://doi.org/10.1186/s12891-018-2256-7

60. Gross, K. D., \& Hillstrom, H. J. (2008). Noninvasive devices targeting the mechanics of osteoarthritis. Rheum Dis Clin North Am, 34(3), 755-776. https://doi.org/10.1016/j.rdc.2008.06.001

61. Ramsey, D. K., \& Russell, M. E. (2009). Unloader braces for medial compartment knee osteoarthritis: implications on mediating progression. Sports Health, 1(5), 416-426. https://doi.org/10.1177/1941738109343157

62. Budarick, A. R., MacKeil, B. E., Fitzgerald, S., \& Cowper-Smith, C. D. (2019). Design Evaluation of a Novel Multicompartment Unloader Knee Brace. J Biomech Eng, 142(1). https://doi.org/10.1115/1.4044818

63. Kluesner, A. J., \& Wukich, D. K. (2009). Ankle arthrodiastasis. Clin Podiatr Med Surg, 26(2), 227-244. https://doi.org/10.1016/j.cpm.2008.12.006

64. Buckwalter, J. A., Anderson, D. D., Brown, T. D., Tochigi, Y., \& Martin, J. A. (2013). The Roles of Mechanical Stresses in the Pathogenesis of Osteoarthritis: Implications for Treatment of Joint Injuries. Cartilage, 4(4), 286-294. https://doi.org/10.1177/1947603513495889

65. Vito, G. R., and L. Talarico. (2002). Arthrodiastasis of the ankle joint, an alternative to fusion. The proceedings of the Annual Meeting of the Podiatry Institute, 30-34. Retrieved from https://www.yumpu.com/en/document/view/48613077/arthrodiastasis-of-theankle-joint-an-alternative-to-fusion on December 5, 2016.

66. Baumhauer, J. F. (2013). Ankle arthrodesis versus ankle replacement for ankle arthritis. Clin Orthop Relat Res, 471(8), 2439-2442.

https://doi.org/10.1007/s11999-013-3084-6

67. Tellisi, N., Fragomen, A. T., Kleinman, D., O'Malley, M. J., \& Rozbruch, S. R. (2009). Joint preservation of the osteoarthritic ankle using distraction arthroplasty. Foot Ankle Int, 30(4), 318-325.

https://doi.org/10.3113/fai.2009.0318

68. Castagnini, F., Pellegrini, C., Perazzo, L., Vannini, F., \& Buda, R. (2016). Joint sparing treatments in early ankle osteoarthritis: current procedures and future perspectives. Journal of Experimental Orthopaedics, 3(1), 3. https://doi.org/10.1186/s40634-016-0038-4

69. van Valburg, A. A., van Roermund, P. M., Lammens, J., van Melkebeek, J., Verbout, A. J., Lafeber, E. P., \& Bijlsma, J. W. (1995). Can Ilizarov joint distraction delay the need for an arthrodesis of the ankle? A preliminary report. J Bone Joint Surg Br, 77(5), 720-725. https://doi.org/10.1302/0301-620X.77B5.7559696

70. DiDomenico, L. A., \& Gatalyak, N. (2012). End-stage ankle arthritis: arthrodiastasis, supramalleolar osteotomy, or arthrodesis? Clin Podiatr Med Surg, 29(3), 391-412. https://doi.org/10.1016/j.cpm.2012.04.010

71. Alimerzaloo, F., Kashani, R. V., Saeedi, H., Farzi, M., \& Fallahian, N. (2014). Patellar tendon bearing brace: combined effect of heel clearance and ankle status on foot plantar pressure. Prosthet Orthot Int, 38(1), 34-38. https://doi.org/10.1177/0309364613486916 
72. Saltzman, C. L., Johnson, K. A., Goldstein, R. H., \& Donnelly, R. E. (1992). The Patellar Tendon-Bearing Brace as Treatment for Neurotrophic Arthropathy: A Dynamic Force Monitoring Study. Foot and Ankle Clin N Am, 13(1), 14-21. https://doi.org/10.1177/107110079201300103

73. Wanamaker, A. B. (2013). Biomechanical comparison of lower limb unloading between common modalities of ankle foot orthoses. Retrieved from https://digitalcommons.du.edu/etd/685/ on December 5, 2016.

74. Aita, D., Bhave, A., Herzenberg, J. E., Paley, D., \& Cannada, L. (1998). The load applied to the foot in a patellar ligament-bearing cast. J Bone Joint Surg Am, 80(11), 1597-1602. https://doi.org/10.2106/00004623-199811000-00005

75. Tanaka, H., Nagata, K., Goto, T., Hoshiko, H., \& Inoue, A. (2000). The effect of the patellar tendon-bearing cast on loading. J Bone Joint Surg Br, 82(2), 228232. https://doi.org/10.1302/0301-620x.82b2 .9443

76. DiAngelo, D. J., Chung, C. L., \& Parker, M. S. (2018). United States Patent Application Serial No. 62/481,741 and PCT International Application Serial No. PCT/US2018/026248 “DYNAMIC ANKLE ORTHOSIS DEVICES, SYSTEMS, AND METHODS" Located at https://patents.google.com/patent/WO2018187569A1/en

77. Chung, C. L. \& DiAngelo, D. J. (2018). Design and validation testing of a dynamic ankle orthosis. MOJ App Bio Biomech, 2(3), 210-215. https://doi.org/10.15406/mojabb.2018.02.00069

78. Chung, C. L., DiAngelo, D. J., Powell, D. W., \& Paquette, M. R. (2020). Biomechanical Comparison of a New Dynamic Ankle Orthosis to a Standard Ankle-Foot Orthosis During Walking. J Biomech Eng, 142(5). https://doi.org/10.1115/1.4045549

79. Kay, A. D., \& Blazevich, A. J. (2009). Moderate-duration static stretch reduces active and passive plantar flexor moment but not Achilles tendon stiffness or active muscle length. J Appl Physiol (1985), 106(4), 1249-1256.

https://doi.org/10.1152/japplphysiol.91476.2008

80. Sadeghi, H., Sadeghi, S., Prince, F., Allard, P., Labelle, H., \& Vaughan, C. L. (2001). Functional roles of ankle and hip sagittal muscle moments in ablebodied gait. Clin Biomech (Bristol, Avon), 16(8), 688-695. https://doi.org/10.1016/s0268-0033(01)00058-4

81. Fullenkamp, A. M., Tolusso, D. V., Laurent, C. M., Campbell, B. M., \& Cripps, A. E. (2018). A Comparison of Both Motorized and Nonmotorized Treadmill Gait Kinematics to Overground Locomotion. J Sport Rehabil, 27(4), 357-363. https://doi.org/10.1123/jsr.2016-0125

82. Fellin, R. E., Manal, K., \& Davis, I. S. (2010). Comparison of lower extremity kinematic curves during overground and treadmill running. J Appl Biomech, 26(4), 407-414. https://doi.org/10.1123/jab.26.4.407

83. Damiano, D. L., Norman, T., Stanley, C. J., \& Park, H. S. (2011). Comparison of elliptical training, stationary cycling, treadmill walking and overground walking. Gait Posture, 34(2), 260-264. https://doi.org/10.1016/j.gaitpost.2011.05.010 
84. Lee, S. J., \& Hidler, J. (2008). Biomechanics of overground vs. treadmill walking in healthy individuals. J Appl Physiol (1985), 104(3), 747-755. https://doi.org/10.1152/japplphysiol.01380.2006

85. Nagano, H., Begg, R. K., Sparrow, W. A., \& Taylor, S. (2013). A comparison of treadmill and overground walking effects on step cycle asymmetry in young and older individuals. J Appl Biomech, 29(2), 188-193.

https://doi.org/10.1123/jab.29.2.188

86. Malatesta, D., Canepa, M., \& Menendez Fernandez, A. (2017). The effect of treadmill and overground walking on preferred walking speed and gait kinematics in healthy, physically active older adults. Eur J Appl Physiol, 117(9), 1833-1843. https://doi.org/10.1007/s00421-017-3672-3

87. Browning, R. C., Baker, E. A., Herron, J. A., \& Kram, R. (2006). Effects of obesity and sex on the energetic cost and preferred speed of walking. J Appl Physiol (1985), 100(2), 390-398.

https://doi.org/10.1152/japplphysiol.00767.2005

88. Levine, R. V., \& Norenzayan, A. (1999). The Pace of Life in 31 Countries. Journal of Cross-Cultural Psychology, 30(2), 178-205. https://doi.org/10.1177/0022022199030002003

89. Zeni, J. A., Jr., Richards, J. G., \& Higginson, J. S. (2008). Two simple methods for determining gait events during treadmill and overground walking using kinematic data. Gait Posture, 27(4), 710-714. https://doi.org/10.1016/j.gaitpost.2007.07.007

90. Baumhauer, J. F., Wervey, R., McWilliams, J., Harris, G. F., \& Shereff, M. J. (1997). A comparison study of plantar foot pressure in a standardized shoe, total contact cast, and prefabricated pneumatic walking brace. Foot Ankle Int, 18(1), 26-33. https://doi.org/10.1177/107110079701800106

91. Hartsell, H. D., Fellner, C., \& Saltzman, C. L. (2001). Pneumatic Bracing and Total Contact Casting Have Equivocal Effects on Plantar Pressure Relief. Foot and Ankle International, 22(6), 502-506. https://doi.org/10.1177/107110070102200609

92. Guillebastre, B., Calmels, P., \& Rougier, P. (2009). Effects of Rigid and Dynamic Ankle-Foot Orthoses on Normal Gait. Foot and Ankle International, 30(1), 51-56. https://doi.org/10.3113/FAI.2009.0051

93. Ramakrishnan, T., Lahiff, C. A., \& Reed, K. B. (2018). Comparing Gait with Multiple Physical Asymmetries Using Consolidated Metrics. Front Neurorobot, 12, 2. https://doi.org/10.3389/fnbot.2018.00002

94. Donker, S. F., Mulder, T., Nienhuis, B., \& Duysens, J. (2002). Adaptations in arm movements for added mass to wrist or ankle during walking. Exp Brain Res, 146(1), 26-31. https://doi.org/10.1007/s00221-002-1145-2

95. Nicolette, G. W., Edenfield, K. M., Michaudet, C., \& Carek, P. J. (2018). Foot and Ankle Conditions: Chronic Lateral Ankle Pain. FP Essent, 465, 24-29. https://www.ncbi.nlm.nih.gov/pubmed/29381042

96. Saltzman, C. L., Salamon, M. L., Blanchard, G. M., Huff, T., Hayes, A., Buckwalter, J. A., \& Amendola, A. (2005). Epidemiology of ankle arthritis: report of a consecutive series of 639 patients from a tertiary orthopaedic center. 
Iowa Orthop J, 25, 44-46.

https://www.ncbi.nlm.nih.gov/pmc/articles/PMC1888779/

97. Hale, S., \& Hertel, J. (2005). Reliability and Sensitivity of the Foot and Ankle

Disability Index in Subjects With Chronic Ankle Instability. Journal of athletic training, 40, 35-40. https://www.ncbi.nlm.nih.gov/pmc/articles/PMC1088343/

98. Martin, R., Burdett, R., \& Irrgang, J. (1999). Development of the Foot and Ankle Disability Index (FADI). Journal of Orthopaedic \& Sports Physical Therapy, 29, A32-A33. Score retrieved from https://www.orthopaedicscore.com/scorepages/foot_and_ankle_disability index fadi.html on August 2, 2018.

99. Riebel, M., Crowell, M., Dolbeer, J., Szymanek, E., \& Goss, D. (2017). Correlation of self-reported outcome measures and the selective functional movement assessment (SFMA): an exploration of validity. Int J Sports Phys Ther, 12(6), 931-947. https://doi.org/10.26603/ijspt20170931

100. Teyhen, D. S., Shaffer, S. W., Lorenson, C. L., Halfpap, J. P., Donofry, D. F., Walker, M. J., \& Childs, J. D. (2012). The Functional Movement Screen: a reliability study. J Orthop Sports Phys Ther, 42(6), 530-540. https://doi.org/10.2519/jospt.2012.3838

101. Butland, R. J., Pang, J., Gross, E. R., Woodcock, A. A., \& Geddes, D. M. (1982). Two-, six-, and 12-minute walking tests in respiratory disease. British medical journal (Clinical research ed.), 284(6329), 1607-1608. https://doi.org/10.1136/bmj.284.6329.1607

102. Rossier, P., \& Wade, D. T. (2001). Validity and reliability comparison of 4 mobility measures in patients presenting with neurologic impairment. Arch Phys Med Rehabil, 82(1), 9-13. https://doi.org/10.1053/apmr.2001.9396

103. Burns, G. T., Zendler, J. D., \& Zernicke, R. F. (2018). Validation of a wireless shoe insole for ground reaction force measurement. Journal of Sports Sciences, 37(10), 1129-1138. https://doi.org/10.1080/02640414.2018.1545515

104. Seiberl, W., Jensen, E., Merker, J., Leitel, M., \& Schwirtz, A. (2018). Accuracy and precision of loadsol ${ }^{\circledR}$ insole force-sensors for the quantification of ground reaction force-based biomechanical running parameters. Eur J Sport Sci, 18(8), 1100-1109. https://doi.org/10.1080/17461391.2018.1477993

105. Renner, K. E., Williams, D. B., \& Queen, R. M. (2019). The Reliability and Validity of the Loadsol $\AA^{\circledR}$ under Various Walking and Running Conditions. Sensors, 19(2), 265. https://doi.org/10.3390/s19020265

106. Mundermann, A., Dyrby, C. O., \& Andriacchi, T. P. (2005). Secondary gait changes in patients with medial compartment knee osteoarthritis: increased load at the ankle, knee, and hip during walking. Arthritis Rheum, 52(9), 2835-2844. https://doi.org/10.1002/art.21262

107. Ewers, B. J., Jayaraman, V. M., Banglmaier, R. F., \& Haut, R. C. (2002). Rate of blunt impact loading affects changes in retropatellar cartilage and underlying bone in the rabbit patella. J Biomech, 35(6), 747-755. https://doi.org/10.1016/s0021-9290(02)00019-2

108. Kerin, A. J., Coleman, A., Wisnom, M. R., \& Adams, M. A. (2003). Propagation of surface fissures in articular cartilage in response to cyclic 
loading in vitro. Clin Biomech (Bristol, Avon), 18(10), 960-968. https://doi.org/10.1016/j.clinbiomech.2003.07.001

109. Kelly, A. M. (1998). Does the clinically significant difference in visual analog scale pain scores vary with gender, age, or cause of pain? Acad Emerg Med, 5(11), 1086-1090. https://doi.org/10.1111/j.1553-2712.1998.tb02667.x

110. Todd, K. H., Funk, K. G., Funk, J. P., \& Bonacci, R. (1996). Clinical significance of reported changes in pain severity. Ann Emerg Med, 27(4), 485489. https://doi.org/10.1016/s0196-0644(96)70238-x

111. Bird, S. B., \& Dickson, E. W. (2001). Clinically significant changes in pain along the visual analog scale. Ann Emerg Med, 38(6), 639-643. https://doi.org/10.1067/mem.2001.118012

112. Kelly, A. M. (2001). The minimum clinically significant difference in visual analogue scale pain score does not differ with severity of pain. Emergency medicine journal : EMJ, 18(3), 205-207. https://doi.org/10.1136/emj.18.3.205

113. Landorf, K. B., Radford, J. A., \& Hudson, S. (2010). Minimal Important Difference (MID) of two commonly used outcome measures for foot problems. Journal of Foot and Ankle Research, 3, 7-7. https://doi.org/10.1186/1757-1146$\underline{3-7}$

114. Landorf, K. B., \& Radford, J. A. (2008). Minimal important difference: Values for the Foot Health Status Questionnaire, Foot Function Index and Visual Analogue Scale. The Foot, 18(1), 15-19. https://doi.org/10.1016/j.foot.2007.06.006

115. Sutton, R. M., McDonald, E. L., Shakked, R. J., Fuchs, D., \& Raikin, S. M. (2019). Determination of Minimum Clinically Important Difference (MCID) in Visual Analog Scale (VAS) Pain and Foot and Ankle Ability Measure (FAAM) Scores After Hallux Valgus Surgery. Foot Ankle Int, 40(6), 687-693. https://doi.org/10.1177/1071100719834539

116. Bubra, P. S., Keighley, G., Rateesh, S., \& Carmody, D. (2015). Posterior tibial tendon dysfunction: an overlooked cause of foot deformity. J Family Med Prim Care, 4(1), 26-29. https://doi.org/10.4103/2249-4863.152245

117. Nitra Pneumatics. (2020) A-Series Pneumatic Cylinders 9/16 inch Bore Specification Sheets: A09040DD. Retrieved from https://cdn.automationdirect.com/static/specs/nitraca916.pdf on January 24, 2020.

118. McMaster-Carr. (2020) Swivel-Shaft Rod End with Set Screw, Nut and Washer, 10-32 Thread, 1-1/16" Center Length: 6154K11. Retrieved from https://www.mcmaster.com/6154K11 on January 24, 2020.

119. Igus. (2020) Rod end with female thread, KBLI igubal ${ }^{\circledR}$, spherical ball iglide ${ }^{\circledR}$ L280, inch: KBLI-03. Retrieved from https://www.igus.com/product/208 on January 24, 2020.

120. JR3 Multi-Axis Load Cell Technologies. (2015) Specification Sheets: 100M40A3.2015; Retrieved from http://www.jr3.com/specification-sheets.html on October 31, 2015.

121. Exlar Actuation Solutions. (2015) Product Specifications: GSX30 Series. Retrieved from http://exlar.com/product/gsx-series/specs on October 31, 2015. 
122. Novel Electronics. (2020) pedar®: Dynamic pressure distribution inside the footwear. Retrieved from https://www.novel.de/products/pedar/ on January 24, 2020.

123. Qualisys AB. (2011) Qualisys Track Manager User Manual. Retrieved from https://www.qualisys.com/hardware/5-6-7/ on January 24, 2020.

124. Novel Electronics. (2020) loadsol®: plantar normal force inside footwear. Retrieved from https://www.novel.de/products/loadsol/ on January 16, 2020.

125. Lathrop-Lambach, R. L., Asay, J. L., Jamison, S. T., Pan, X., Schmitt, L. C., Blazek, K., \& Chaudhari, A. M. (2014). Evidence for joint moment asymmetry in healthy populations during gait. Gait Posture, 40(4), 526-531. https://doi.org/10.1016/j.gaitpost.2014.06.010

126. Lin, P. Y., Yang, Y. R., Cheng, S. J., \& Wang, R. Y. (2006). The relation between ankle impairments and gait velocity and symmetry in people with stroke. Arch Phys Med Rehabil, 87(4), 562-568. https://doi.org/10.1016/j.apmr.2005.12.042

127. Patterson, K. K., Parafianowicz, I., Danells, C. J., Closson, V., Verrier, M. C., Staines, W. R., \& McIlroy, W. E. (2008). Gait asymmetry in communityambulating stroke survivors. Arch Phys Med Rehabil, 89(2), 304-310. https://doi.org/10.1016/j.apmr.2007.08.142

128. Gulgin, H., Hall, K., Luzadre, A., \& Kayfish, E. (2018). 3D gait analysis with and without an orthopedic walking boot. Gait Posture, 59, 76-82. https://doi.org/10.1016/j.gaitpost.2017.09.024

129. Jafarnezhadgero, A., Madadi Shad, M., \& Ferber, R. (2018). The effect of foot orthoses on joint moment asymmetry in male children with flexible flat feet. $\mathrm{J}$ Bodyw Mov Ther, 22(1), 83-89. https://doi.org/10.1016/j.jbmt.2017.04.007

130. Esquenazi, A., Ofluoglu, D., Hirai, B., \& Kim, S. (2009). The effect of an ankle-foot orthosis on temporal spatial parameters and asymmetry of gait in hemiparetic patients. PM R, 1(11), 1014-1018. https://doi.org/10.1016/j.pmrj.2009.09.012

131. Mills, K., Hettinga, B. A., Pohl, M. B., \& Ferber, R. (2013). Between-limb kinematic asymmetry during gait in unilateral and bilateral mild to moderate knee osteoarthritis. Arch Phys Med Rehabil, 94(11), 2241-2247. https://doi.org/10.1016/j.apmr.2013.05.010 


\section{APPENDIX A. SPECIFICATIONS}

This appendix provides specifications of the DAO components (Table A-1) and equipment (Table A-2) used throughout this body of work.

Table A-1. DAO Component Specifications

Cylinder body material: $304 \mathrm{SS}$
Piston rod material: $303 \mathrm{SS}$
End caps material: High strength aluminum
0.25 sq. in. piston area
Pressure rating $250 \mathrm{psi}$
Extend force at $100 \mathrm{psi}=25.0 \mathrm{lb}$
Retract force at $100 \mathrm{psi}=22.2 \mathrm{lb}$
Body length: $6.56 \mathrm{in}$
Stroke length: 4.0 in
Material: Zinc-Plated Carbon Steel
Maximum Ball Swivel: $30^{\circ}$
Shank Thread Size: $10-32$
Shank Thread Length: $1 / 4 "$

Sources: Nitra Pneumatics. (2020) A-Series Pneumatic Cylinders 9/16 inch Bore Specification Sheets: A09040DD. Retrieved from https://cdn.automationdirect.com/static/specs/nitraca916.pdf on January 24, 2020. [117] McMaster-Carr. (2020) Swivel-Shaft Rod End with Set Screw, Nut and Washer, 10-32 Thread, 1-1/16" Center Length: 6154K11. Retrieved from https://www.mcmaster.com/6154K11 on January 24, 2020. [118] Igus. (2020) Rod end with female thread, KBLI igubal ${ }^{\circledR}$, spherical ball iglide ${ }^{\circledR}$ L280, inch: KBLI-03. Retrieved from https://www.igus.com/product/208 on January 24, 2020. [119] 
Table A-2. Equipment Specifications

\begin{tabular}{|c|c|}
\hline System Information & Specifications \\
\hline $\begin{array}{l}\text { Load Cell } \\
\text { JR3 Multi-Axis Force-Torque } \\
\text { Sensor } \\
\text { Model: 100M40A3 } \\
\text { Mechanical Load Rating: } 400 \mathrm{~N}\end{array}$ & $\begin{array}{l}\text { X-Axis and Y-Axis Force Readings } \\
\text { Standard Measurement Range: } \pm 400 \mathrm{~N} \\
\text { Standard Resolution: } 0.10 \mathrm{~N} \\
\text { Single-Axis Maximum Load: } 2000 \mathrm{~N} \\
\text { Z-Axis Force Readings } \\
\text { Standard Measurement Range: } \pm 800 \mathrm{~N} \\
\text { Standard Resolution: } 0.20 \mathrm{~N} \\
\text { Single-Axis Maximum Load: } 8750 \mathrm{~N}\end{array}$ \\
\hline $\begin{array}{l}\text { Vertical Actuator } \\
\text { Exlar GSX30-1201-OFM-CS2-138- } \\
\text { AR } \\
\text { Serial No.: 04130798 } \\
\text { P/N: } 22749 \text { Rev: A }\end{array}$ & $\begin{array}{l}\text { Volts: } 230 \\
\text { Amps: } 2.1 \\
\text { RPM: } 3000 \\
\text { Frame Size: } 3.125 \mathrm{in}(79 \mathrm{~mm}) \\
\text { Stroke: } 12 \text { in }(305 \mathrm{~mm}) \\
\text { Screw Lead: } 0.1 \mathrm{in}(2.54 \mathrm{~mm}) \\
\text { Max Velocity: } 5 \mathrm{in} / \mathrm{sec}(127 \mathrm{~mm} / \mathrm{sec}) \\
\text { Maximum Static Load: } 2700 \mathrm{lbs}(12010 \mathrm{~N}) \\
\text { Armature Inertia: } 0.00443 \mathrm{lb}-\mathrm{in}-\mathrm{s} 2(0.000501 \mathrm{~kg}- \\
\text { m2) } \\
\text { Dynamic Load Rating: } 5516 \mathrm{lbs}(24536 \mathrm{~N}) \\
\text { Weight: } 20.5 \mathrm{lbs}(9.3 \mathrm{~kg})\end{array}$ \\
\hline $\begin{array}{l}\text { Pedar insoles } \\
\text { Various sizes }\end{array}$ & $\begin{array}{l}\text { Sampling frequency: up to } 200 \mathrm{~Hz} \\
99 \text { sensors per insole } \\
\text { Thickness }(\mathrm{mm}): 1.9 \\
\text { Number of sensors: } 85-99 \\
\text { Pressure range }(\mathrm{kPa}): 15-600 \text { or } 30-1,200 \\
\text { Resolution }(\mathrm{kPa}): 2.5 \text { or } 5\end{array}$ \\
\hline $\begin{array}{l}\text { Qualysis cameras } \\
\text { Oqus } 3\end{array}$ & $\begin{array}{l}\text { Sampling frequency: } 240 \mathrm{~Hz} \\
\text { Full resolution of } 1280 \times 1024 \\
\text { Normal mode (full FOV): } 1.3 \mathrm{MP}, 500 \mathrm{fps} \\
\text { High-speed mode (full FOV): } 0.3 \mathrm{MP}, 1750 \mathrm{fps} \\
\text { Max capture distance: } 22 \mathrm{~mm}\end{array}$ \\
\hline $\begin{array}{l}\text { Loadsol insoles } \\
\text { Various sizes } \\
\text { Serial No: P1X } \\
\text { Version } 1.4 .88\end{array}$ & $\begin{array}{l}\text { Sampling frequency: } 200 \mathrm{~Hz} \\
\text { Force range: } 0-2550 \mathrm{~N} \\
\text { Resolution: } 10 \mathrm{~N} \\
\text { Calibrated accuracy: } \pm 5 \%\end{array}$ \\
\hline
\end{tabular}

Sources: JR3 Multi-Axis Load Cell Technologies. (2015) Specification Sheets:

100M40A3.2015; Retrieved from http://www.jr3.com/specification-sheets.html on October 31, 2015. [120] Exlar Actuation Solutions. (2015) Product Specifications: 


\section{Table A-2. Continued}

GSX30 Series. Retrieved from http://exlar.com/product/gsx-series/specs on October 31,2015. [121] Novel Electronics. (2020) pedar $\AA$ : Dynamic pressure distribution inside the footwear. Retrieved from https://www.novel.de/products/pedar/ on January 24, 2020. [122] Qualisys AB. (2011) Qualisys Track Manager User Manual. Retrieved from https://www.qualisys.com/hardware/5-6-7/ on January 24, 2020. [123] Novel Electronics.

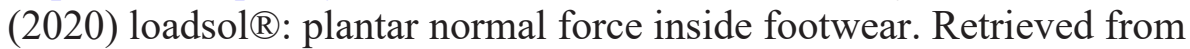
https://www.novel.de/products/loadsol/ on January 16, 2020. [124] 


\section{APPENDIX B. PILOT STUDY COMPARING THE DAO WITH A PTB ORTHOSIS IN OVER-GROUND WALKING}

A brief pilot study was performed to evaluate the effect of DAO and PTB wear on joint kinematics and ground reaction force variables during level over-ground walking. This work helped inform decisions on the methodology and comparative brace that were ultimately used in the work described in Chapter 4.

\section{BACKGROUND}

Reduced axial joint loads have been shown to effectively reduce the pain and discomfort of lower extremity conditions such as ankle osteoarthritis.[70] However, current treatment options like the Patellar Tendon Bearing Ankle-Foot Orthosis (PTB), do not provide a controlled offloading of the ankle joint and tend to limit ankle motion.[17] A new Dynamic Ankle Orthosis (DAO) was designed to provide the coupled benefits of ankle offloading while supporting the natural range of ankle motion. The purpose of this work was to compare ankle joint kinematics and ground reaction force variables between the newly designed DAO and a standard PTB in healthy individuals during level-walking. An unbraced condition (CON) served as the control group (Figure B-1).

\section{METHODS}

Three healthy young women $(22.3 \pm 0.6 \mathrm{yrs} ; 59.8 \pm 9.2 \mathrm{~kg} ; 1.62 \pm 0.07 \mathrm{~m})$ were recruited to participate in this pilot research study. Over-ground walking trials were performed wearing shoes with 1) regular shoe (CON), 2) DAO, and 3) PTB. The DAO was tuned to offload approximately $75 \mathrm{~N}$ of the user's bodyweight. Participants first completed five over-ground walking trials to measure their preferred walking speed in each condition using photocells and an electronic timer (Lafayette Instruments Inc., IN, USA). Anatomical markers and tracking marker clusters were then placed on both lower extremities and a standing calibration trial was collected.

A 9-camera motion capture system $(240 \mathrm{~Hz}$, Qualisys AB, Sweden) and a force platform (1200Hz, AMTI, Inc., USA) were used to collect kinematic and GRF data, respectively. Participants then completed five over-ground level walking trials at a set speed $(1.4 \mathrm{~m} / \mathrm{s} \pm 5 \%)$. Visual3D biomechanical software (C-Motion, Germantown, USA) was used to process and analyze all data. Joint kinetic variables were calculated using Newtonian inverse dynamics. The mean of each variable during the five walking trials was used for analysis. Due to the preliminary nature of work and small sample sizes $(n=3)$, only Cohen's d effect sizes were reported to assess effect magnitudes with Hopkins' interpretation (i.e., small: $d<0.6$, moderate: $0.6 \leq d \leq 1.2$; large: $d>1.2$ ). 

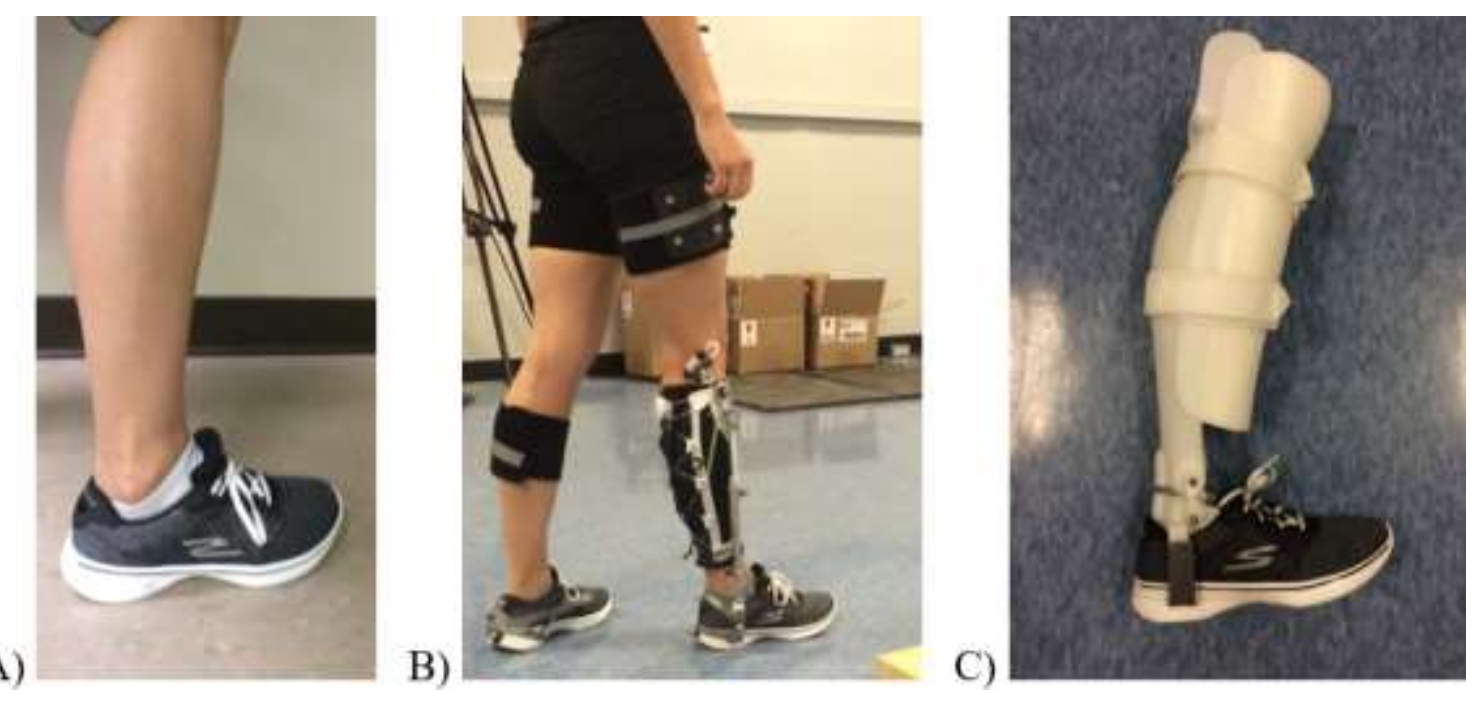

Figure B-1. Bracing Conditions for the Over-Ground Walk Pilot Study A) CON, B) DAO, and C) PTB 


\section{RESULTS AND INTERPRETATION}

Preferred walking speed was not different between $\mathrm{CON}$ and DAO conditions $(d$ $=0.25)$ but was faster in $\mathrm{CON}(d=1.20)$ and DAO $(d=1.66)$ compared to PTB walking; both showing large effect sizes (Table B-1 and Figure B-2). These findings suggest that DAO did not restrict preferred walking speed unlike the standard PTB.

Step length was longer with DAO $(d=0.61)$ and PTB $(d=0.71)$ compared to CON. Peak dorsiflexion was greater in DAO $\left(13.2^{\circ}\right)$ compared to CON $\left(7.7^{\circ}, d=2.92\right)$ and PTB $\left(7.5^{\circ}, d=3.13\right)$ (Figure B-3). Dorsiflexion ROM was larger in DAO $\left(22.1^{\circ}, d=\right.$ $0.94)$ but smaller in PTB $\left(14.1^{\circ}, d=2.02\right)$ compared to the CON $\left(18.9^{\circ}\right)$. Only the PTB and CON difference showed a large effect size. Thus, DAO improved dorsiflexion ROM similar to CON compared to PTB.

Peak eversion was similar between DAO $\left(-3.9^{\circ}\right)$ and $\mathrm{CON}\left(-3.8^{\circ}, d=0.05\right)$, but smaller with PTB $\left(-3.3^{\circ}\right)$ compared to $\mathrm{CON}(d=0.21)$ and DAO $(d=0.54)$ with small effect. Finally, although eversion ROM in $\mathrm{CON}\left(4.85^{\circ}\right)$ was larger compared to DAO $\left(4.05^{\circ}, d=0.52\right)$ and PTB $\left(0.19^{\circ}\right)$, large effects were only observed between PTB and $\mathrm{CON}(d=6.31)$ and DAO $(d=2.87)$. The DAO allowed for greater frontal plane ankle motion than a standard PTB which is more similar to the non-braced condition.

Bracing conditions had negligible effects $(d<0.6)$ on peak horizontal GRF variables (Table B-1). However, vertical loading response and push-off peaks were lower in DAO compared to CON $(d=1.39$ and $d=0.30)$ and PTB $(d=1.42$ and $d=0.67)$. PTB increased the vertical loading response $(d=0.56)$ and push-off peaks $(d=0.49)$ compared to CON. The DAO reduced vertical loading to the body which may promote better tissue recovery during rehabilitation, but more work is needed to fully assess the ankle offloading benefit of the DAO.

\section{CONCLUSIONS}

Findings from this preliminary work demonstrate that the DAO allowed for greater ankle mobility and reduced vertical loading compared to a standard PTB during walking. Therefore, this pilot study supports the need to further investigate the effect of the DAO on ankle movement and loading of the lower extremity. Upon studying the PTB, we learned that the PTB had largely fallen out of practice for treating conditions with mechanical foot and ankle pain. Therefore, the next comparative study evaluating the DAO should assess the standard of care treatment device. Additionally, future work should include a means of evaluating the effect of the DAO on the in-shoe loads, which we hypothesize are more greatly affected than the external GRF measured here, such as with an instrumented insole device. 
Table B-1. Ankle Angular Kinematics and GRF During Walking in Each Bracing Condition

\begin{tabular}{lccc}
\hline Variables & CON & DAO & PTB \\
\hline Preferred Speed $\left(\mathrm{m} \mathrm{s}^{-1}\right)$ & $1.44 \pm 0.08$ & $1.46 \pm 0.07^{\mathrm{b}}$ & $1.37 \pm 0.03$ \\
Step Length (m) & $0.61 \pm 0.03$ & $0.63 \pm 0.03$ & $0.63 \pm 0.03$ \\
Horizontal Lateral Peak (BW) & $0.02 \pm 0.01$ & $0.02 \pm 0.01$ & $0.03 \pm 0.01$ \\
Horizontal Medial Peak (BW) & $-0.05 \pm 0.01$ & $-0.06 \pm 0.01$ & $-0.06 \pm 0.01$ \\
Horizontal Braking Peak (BW) & $-0.14 \pm 0.03$ & $-0.14 \pm 0.03$ & $-0.16 \pm 0.07$ \\
Horizontal Propulsive Peak (BW) & $0.16 \pm 0.06$ & $0.16 \pm 0.05$ & $0.18 \pm 0.09$ \\
Vertical Loading Response Peak (BW) & $0.58 \pm 0.14$ & $0.39 \pm 0.14^{\mathrm{a} b}$ & $0.71 \pm 0.28$ \\
Vertical Push Off Peak (BW) & $0.72 \pm 0.11$ & $0.68 \pm 0.09$ & $0.81 \pm 0.25$ \\
\hline
\end{tabular}

Notes: Mean \pm SD; "a" denotes large effect size vs CON and "b" denotes large effect size vs PTB (large $d>1.2$ ).

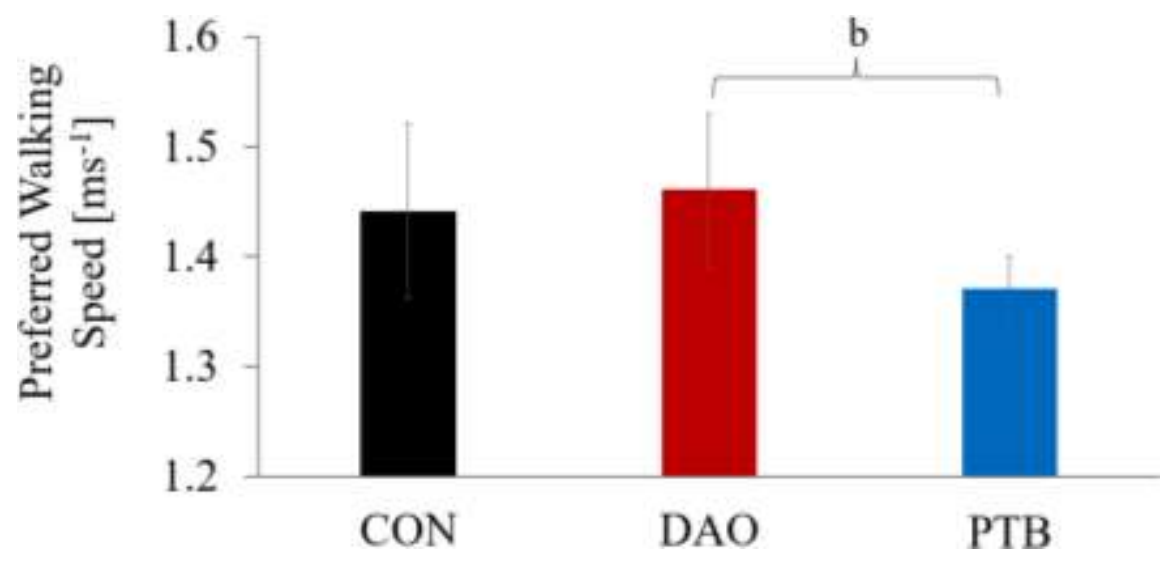

Figure B-2. Preferred Speed for Each Bracing Condition

Mean $\pm \mathrm{SD}$; "b" denotes large effect size vs PTB (large $d>1.2$ ). 


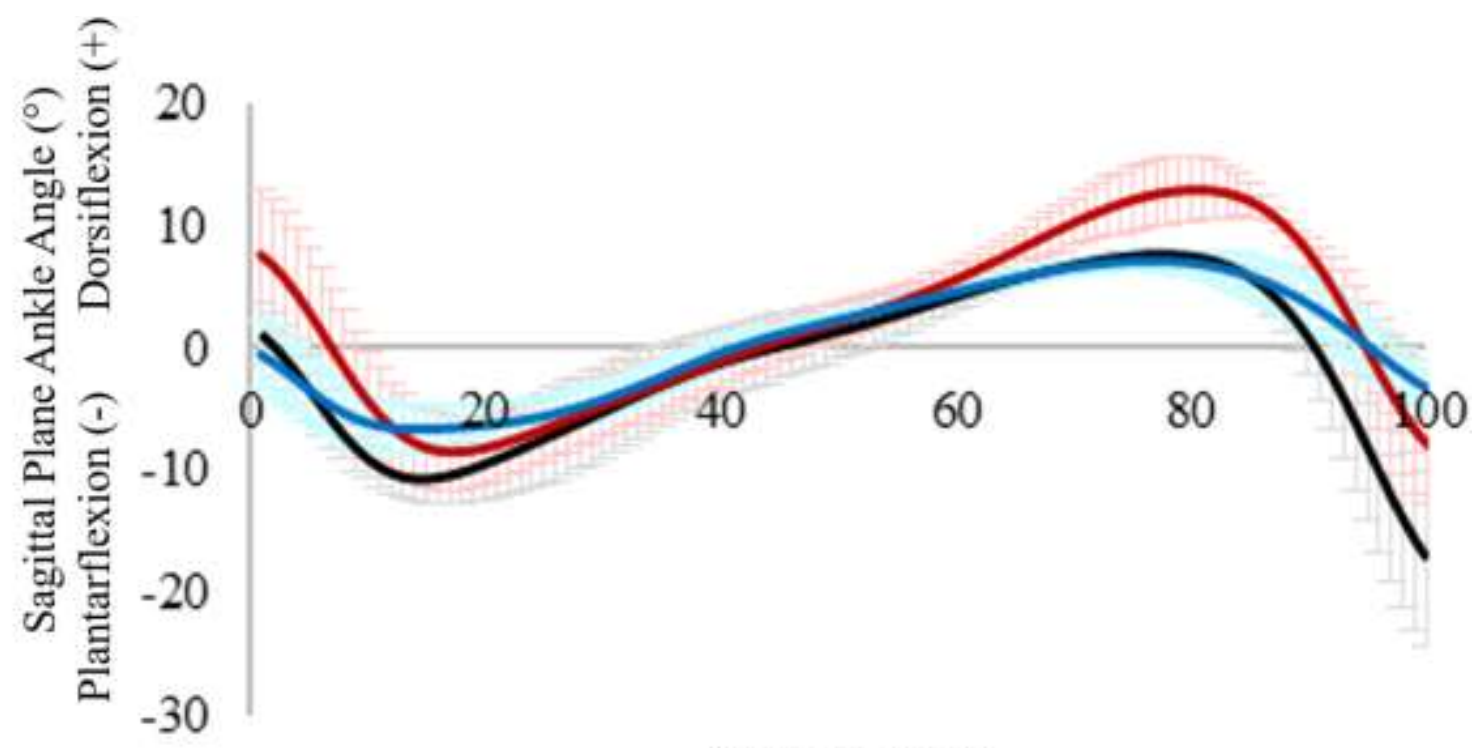

$\%$ Stance Phase

A)
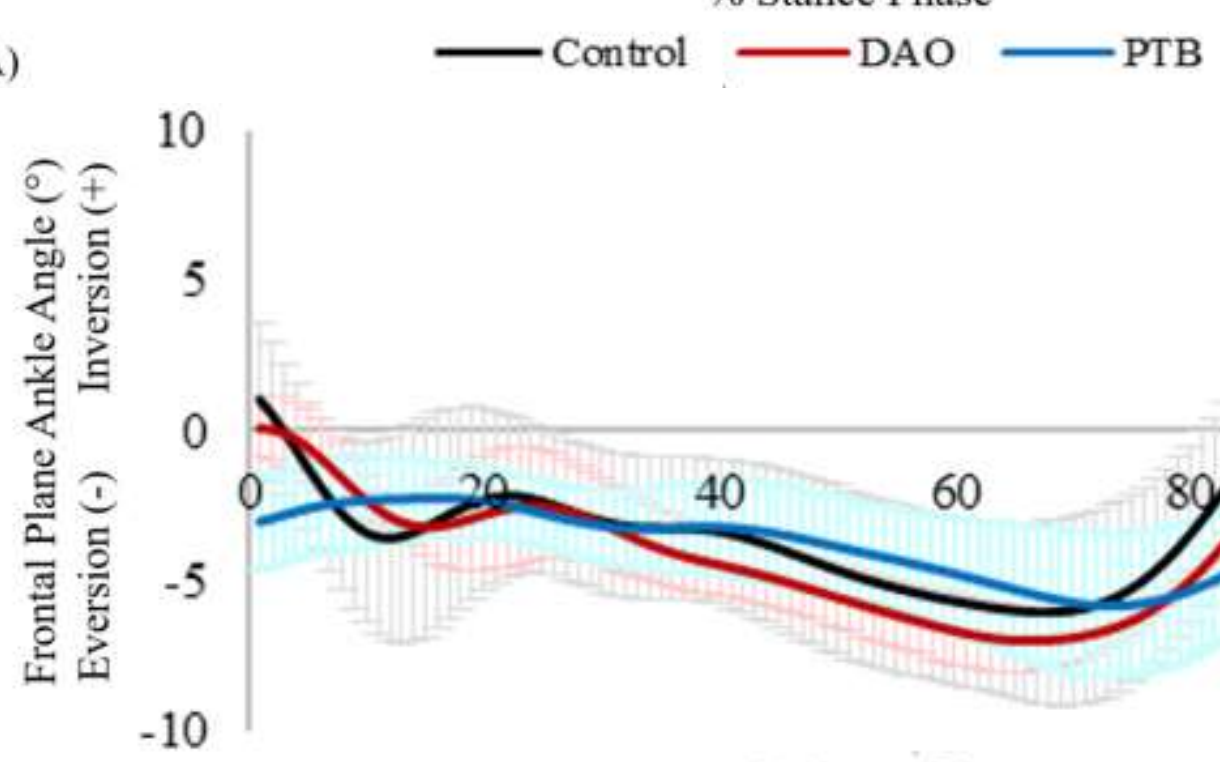

B)

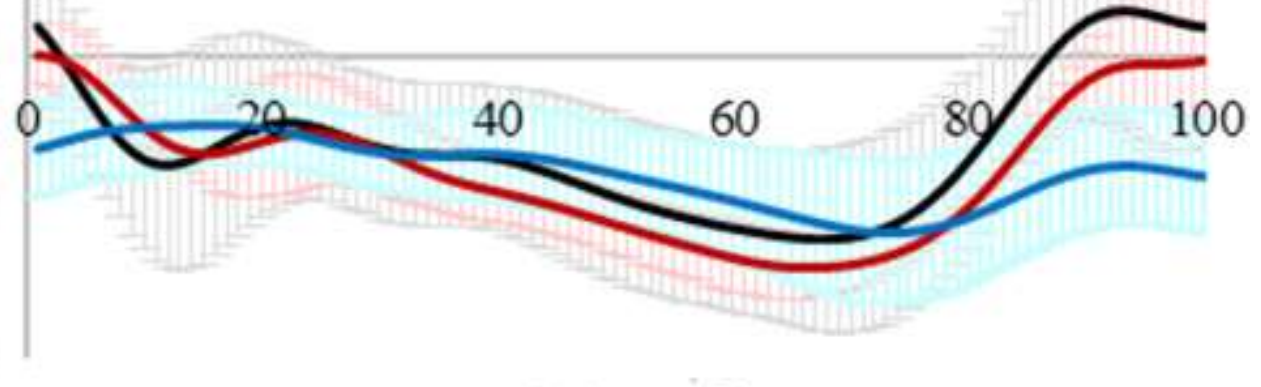

$$
\% \text { Stance Phase }
$$

Control

DAO PTB

Figure B-3. Ankle Angles in the Sagittal Plane and Frontal Plane Mean \pm SD; A) Sagittal Plane and B) Frontal Plane. 


\section{APPENDIX C. EXTENDED METHODOLOGY OF THE FUNCTIONAL ASSESSMENT}

This appendix provides additional information regarding the equipment used and data collected during the functional assessment study described in Chapter 4. Figures C-1 and C-2 show the general equipment setup and marker placement. Figures C-3, C-4, and $\mathbf{C}-\mathbf{5}$ show the treadmill walking activity, sample motion capture data, and sample plantar pressure data.

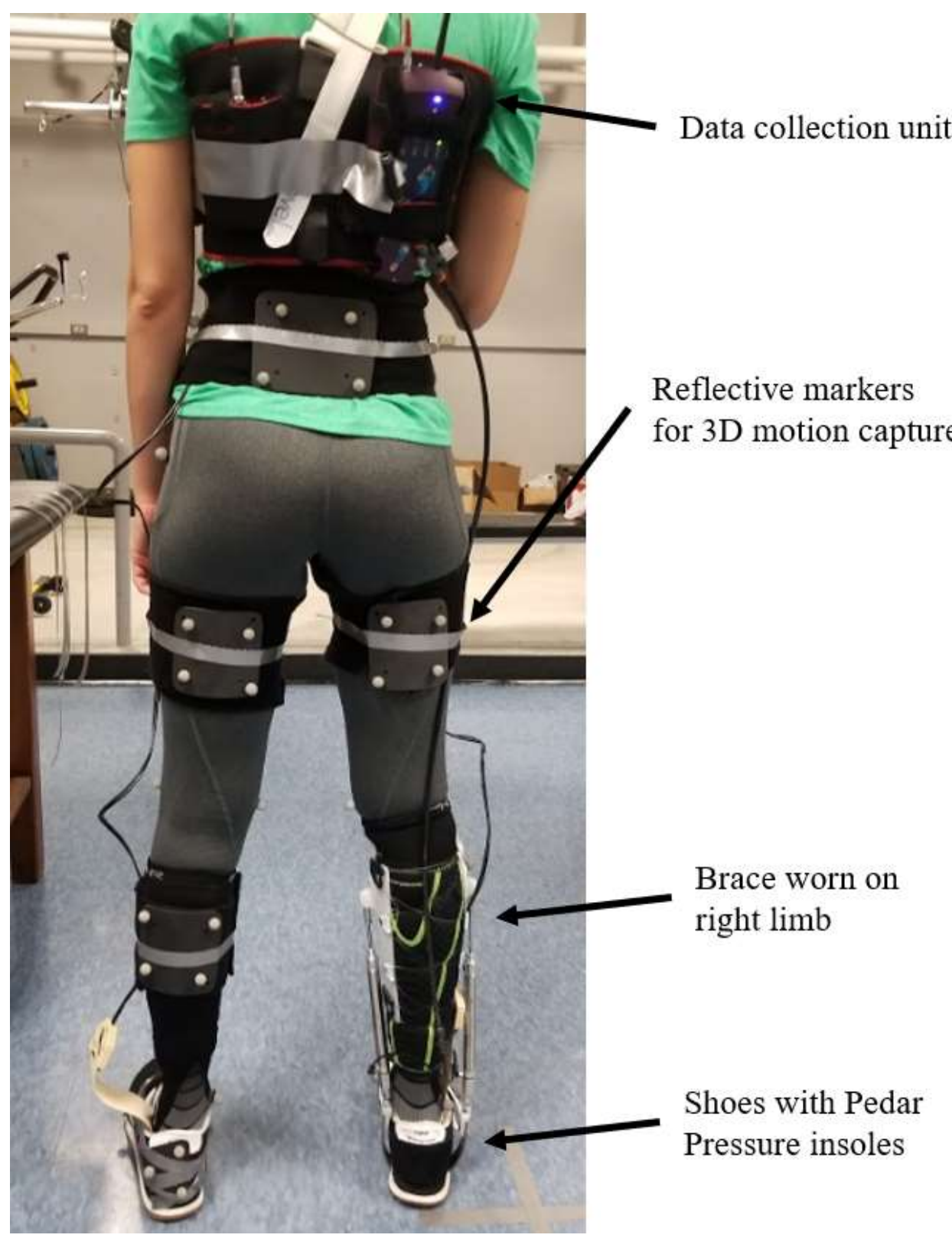

Figure C-1. Equipment and Setup for the Functional Assessment Study 


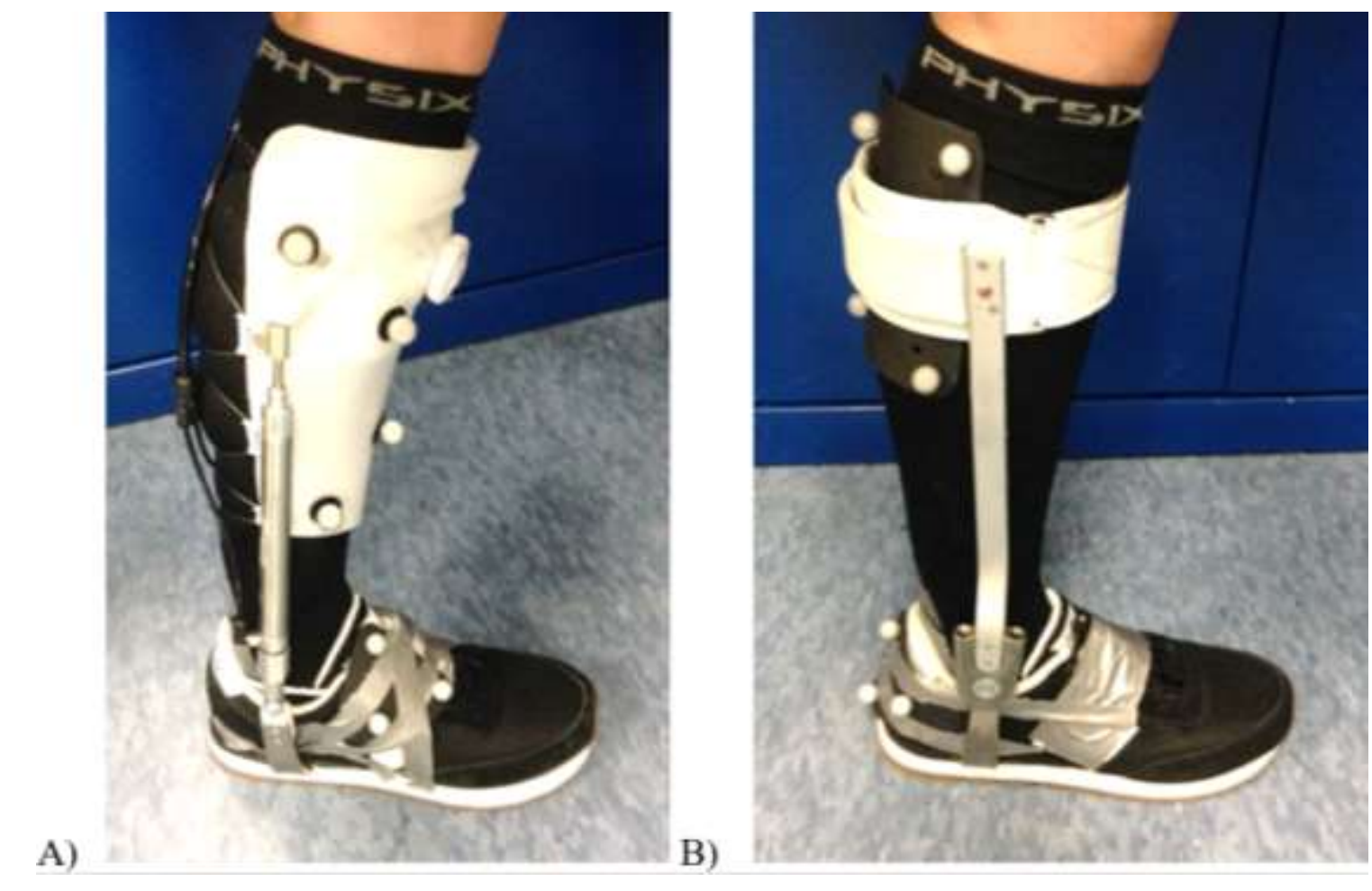

Figure C-2. Reflective Marker Placement for the Bracing Conditions A) DAO and B) DUAFO. 


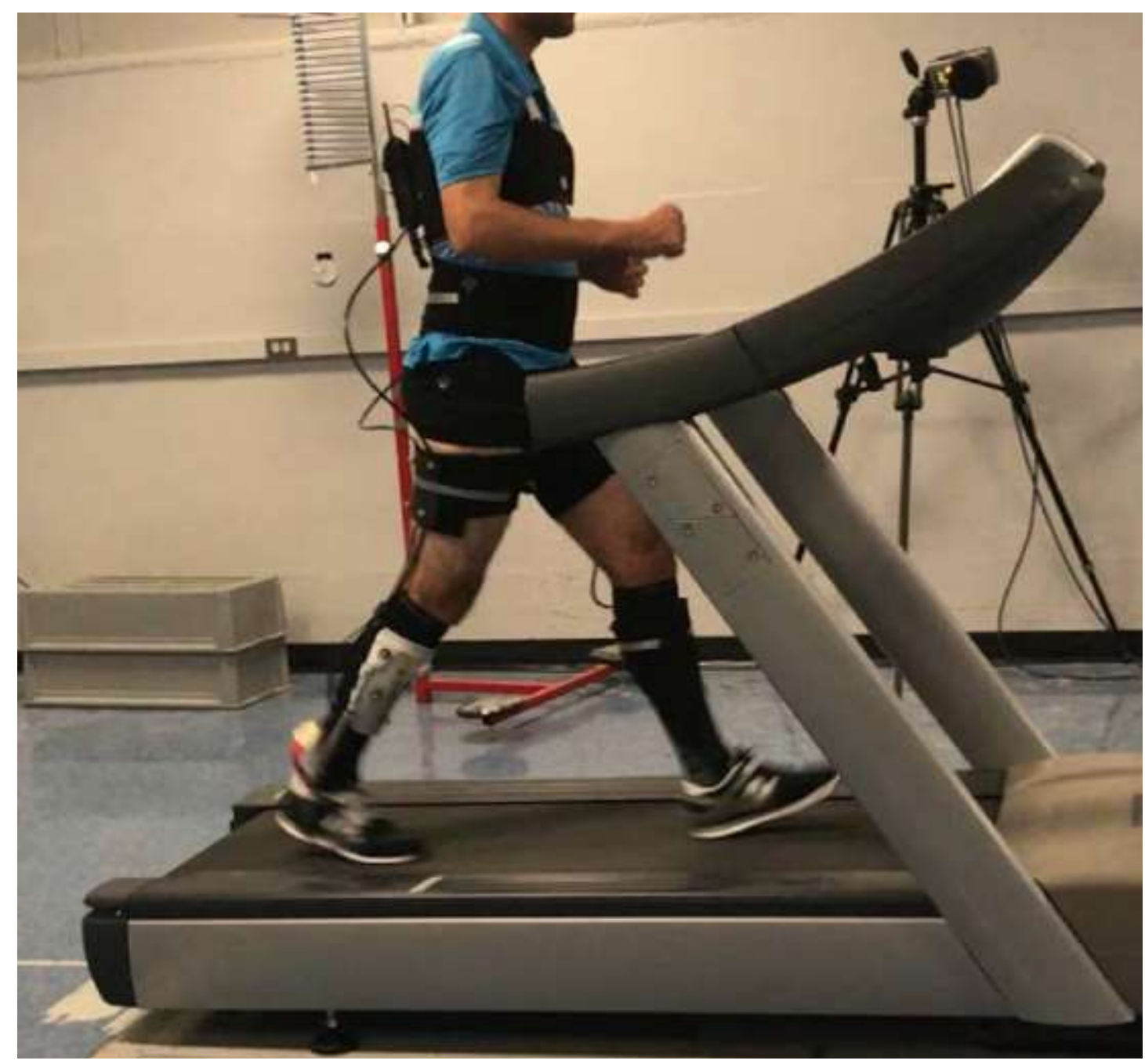

Figure C-3. Treadmill Walking Activity During the Functional Assessment of the DAO 


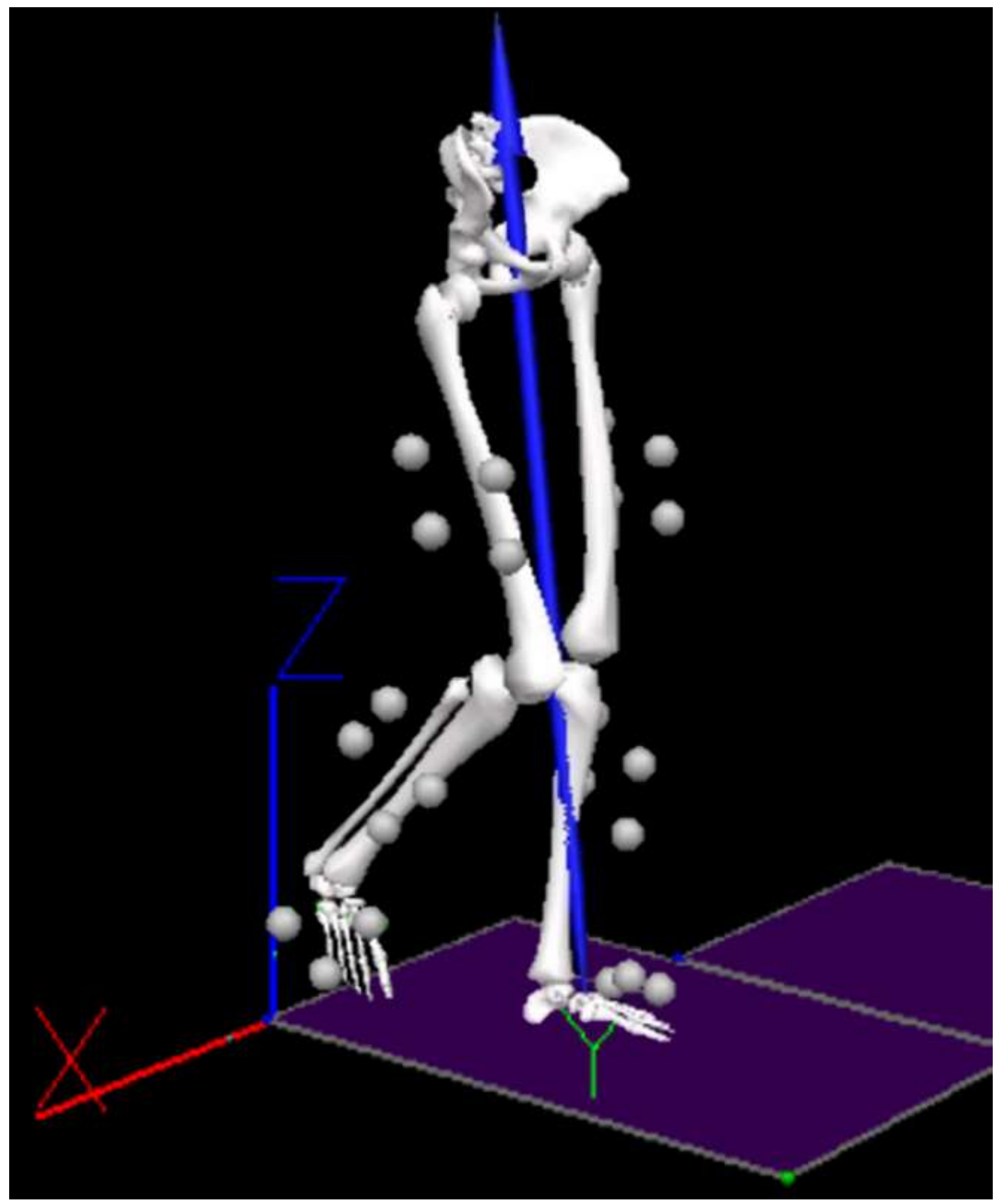

Figure C-4. Sample Visual3D Model Reconstruction of Motion Capture Data During Walking 


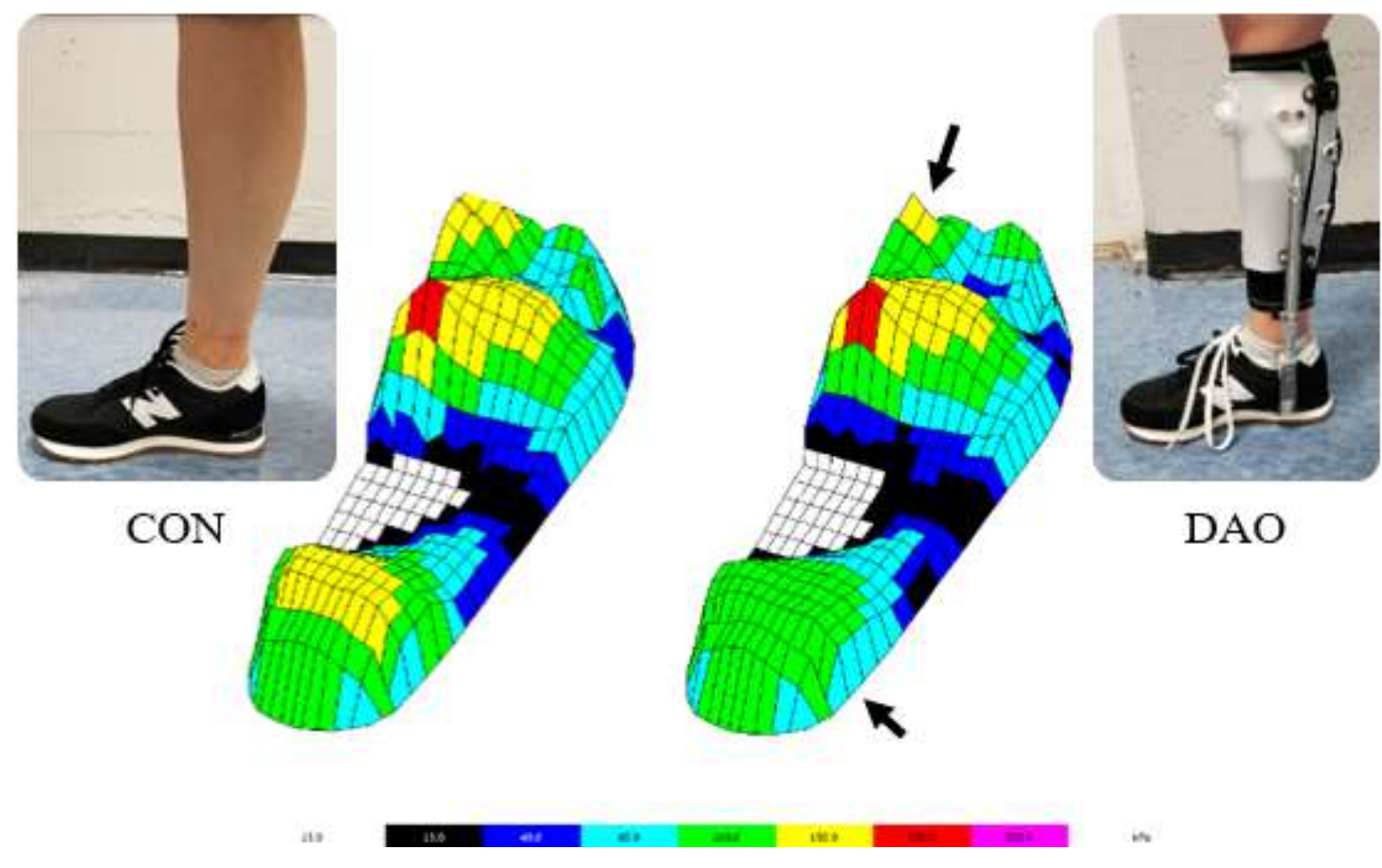

Figure C-5. Sample Plantar Pressure Map for CON and DAO During Walking 


\section{APPENDIX D. EXTENDED RESULTS OF THE FUNCTIONAL ASSESSMENT}

This appendix provides the additional data that was collected during the functional assessment of the DAO described in Chapter 4. During this work, bilateral ankle, knee, and hip joint kinematic data were captured and analyzed. These data were ultimately omitted from the final publication of the work but are included here as an additional reference.

\section{BACKGROUND}

The primary purpose of an AFO is to improve or alter the kinematics and kinetics of the user's ankle-foot complex to treat a disorder or deformity [92,93,125-131]. However, unilateral brace wear can also have an undesired effect and create artificial imbalances between sides during walking. This effect, known as gait asymmetry, is a mechanical deviation of the lower limb during walking. Asymmetry can either occur naturally or result from structural or functional deformities (i.e., leg length discrepancy), diseases that affect motor control (i.e., Stroke) [126,127], or external perturbances (i.e., the presence of a prosthesis or orthosis on one limb) that create an artificial leg length discrepancy or add weight to the end of one limb [128]. The effects of DAO and DUAFO brace wear on ankle, knee, and hip joint motion and walking symmetry were evaluated in the present study using bilateral kinematics and an asymmetry index. We expected to see some compensation occur at the knee and hip joints in the braced conditions in the form of abnormal joint motion relative to $\mathrm{CON}$. Furthermore, we expected that on average, all three conditions (CON, DAO, and DUAFO) would present some level of asymmetry, and we also expected that DAO and DUAFO would present higher levels of asymmetry compared to $\mathrm{CON}$ (since the healthy individuals did not require an orthosis to correct some condition).

\section{METHODS}

In addition to the methods presented in Chapter 4, motion of the ankle, knee, and hip joints were measured bilaterally (Figure D-1, D-2, and D-3) and used to derive various spatio-temporal and joint kinematic variables. An asymmetry index was computed for each variable using Equation D-1.

$$
\text { Asymmetry index }=\left|1-\frac{V_{\text {Braced }}}{V_{\text {Unbraced }}}\right| \times 100 \%
$$

where "V" represents the variable of interest [130], an asymmetry index $=0$ represents no asymmetry, and an asymmetry index $>0$ represents asymmetry. 

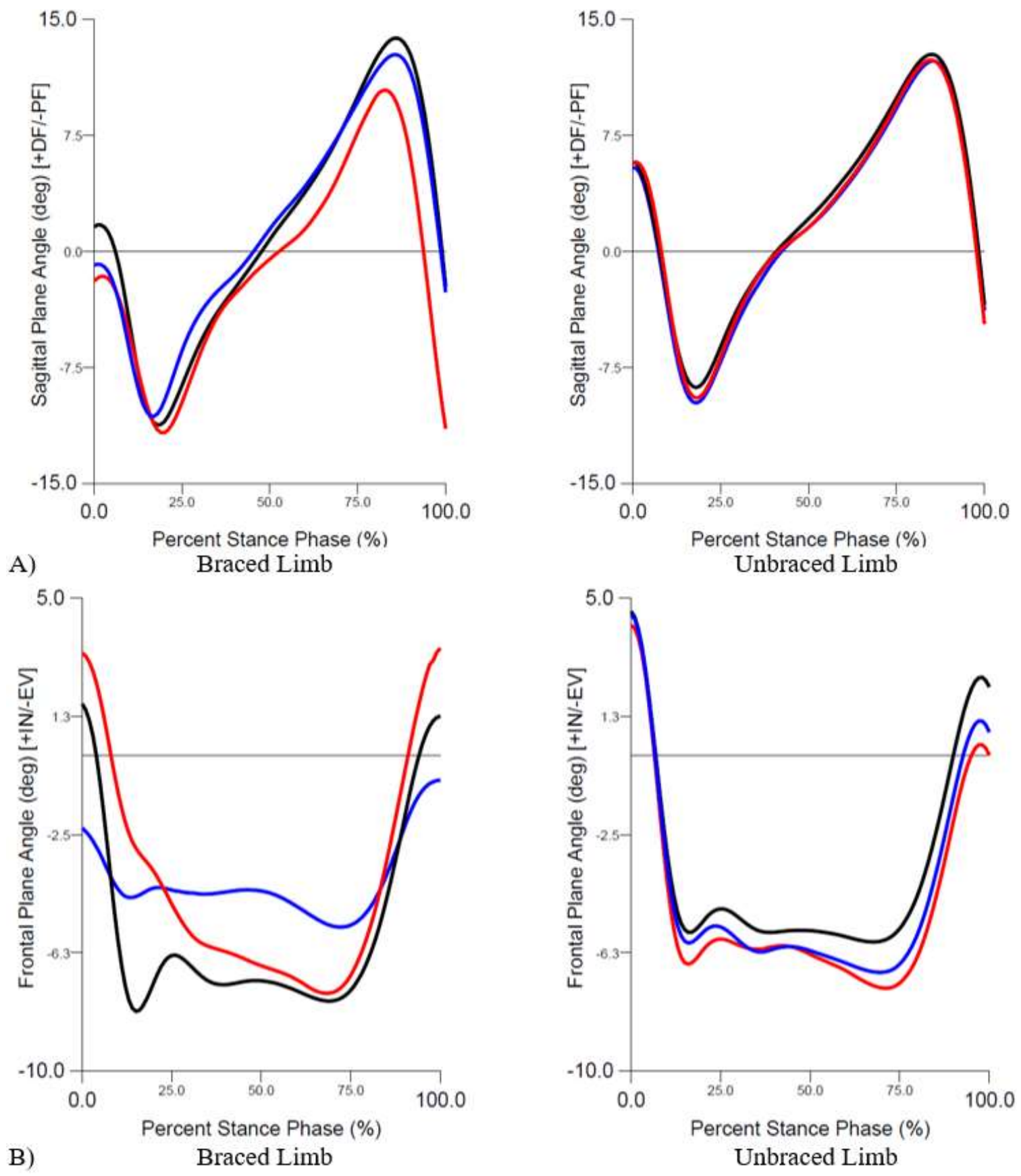

Figure D-1. Ankle Joint Kinematic Ensemble Curves in Sagittal Plane and Frontal Plane for the Braced and Unbraced Limbs

A) Sagittal Plane and B) Frontal Plane; Black: CON, Red: DAO, and Blue: DUAFO. 

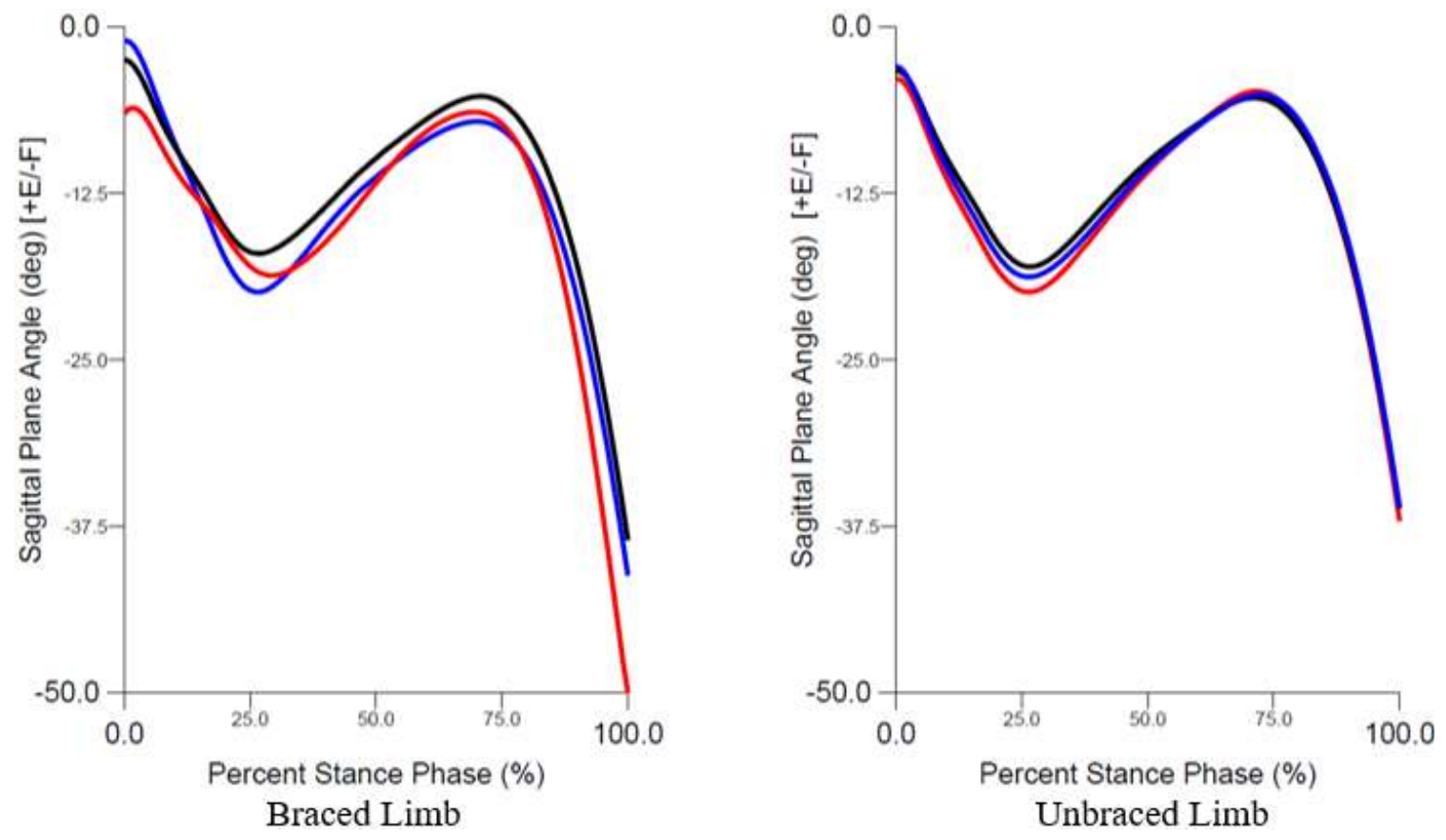

Figure D-2. Knee Joint Kinematic Ensemble Curves in Sagittal Plane for the Braced and Unbraced Limbs

Black: CON, Red: DAO, and Blue: DUAFO. 

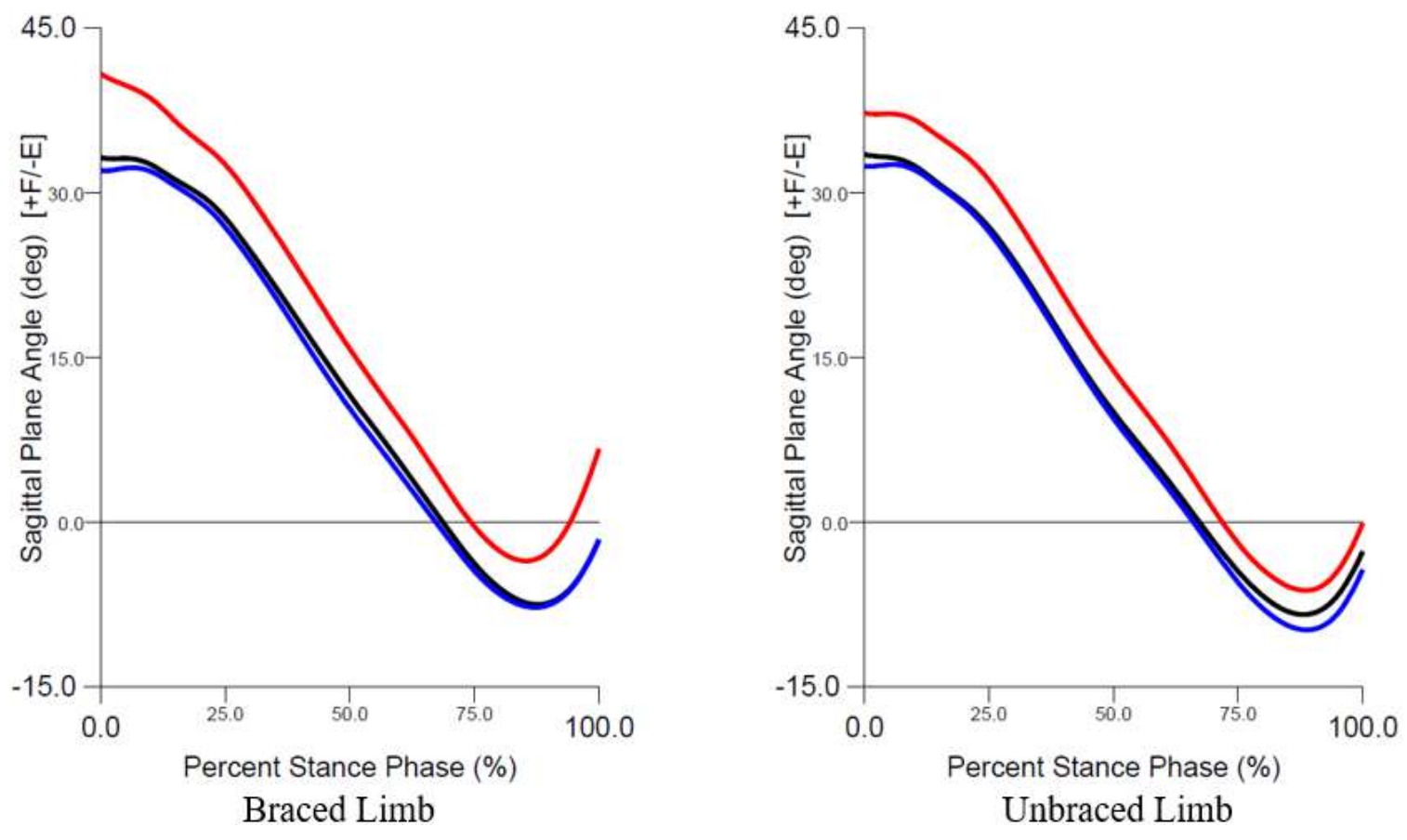

Figure D-3. Hip Joint Kinematic Ensemble Curves in Sagittal Plane for the Braced and Unbraced Limbs

Black: CON, Red: DAO, and Blue: DUAFO. 


\section{RESULTS AND INTERPRETATION}

Spatio-temporal variables for the braced and unbraced limb are shown in Table D-1. None of the spatio-temporal variables were found to be asymmetric (asymmetry index $>10$ ) in any of the brace conditions. Both bracing conditions significantly increased the stance time of the unbraced limb compared to CON. DAO increased the asymmetry index of stance time compared to CON and DUAFO, but the parameter remained symmetric. Furthermore, DAO significantly reduces the cadence of the unbraced limb compared to CON and DUAFO.

The ankle joint kinematic variables for the braced and unbraced limb are shown in Table D-2. All of the sagittal plane and frontal plane ankle kinematic variables were asymmetric (asymmetry index $>10$ ) for all three conditions. Early stance peak plantarflexion angular velocity was more asymmetric in DAO compared to CON and DUAFO and more asymmetric in DUAFO compared to CON. This corresponds to the significantly reduced early stance peak plantarflexion angular velocity seen on the braced limb compared to CON and DUAFO. Eversion ROM was more asymmetric in DUAFO compared to $\mathrm{CON}$ and DAO. Early stance peak eversion angular velocity was least asymmetric in CON and most asymmetric in DUAFO.

The knee and hip joint kinematic variables for the braced and unbraced limb are shown in Table D-3. DAO significantly increases the knee sagittal plane contact angle (more flexed) of the braced limb compared to CON and DUAFO. DUAFO significantly increases knee flexion ROM of the braced limb compared to CON. DAO significantly increases the hip sagittal plane contact angle (more flexed) of the braced limb and unbraced limb compared to CON and DUAFO. DAO significantly increases the hip extension ROM of the braced limb compared to CON and DUAFO.

Overall, these findings agree with what we expected. In a study of 182 healthy subjects, it was found that more than half of the subjects naturally presented a clinically relevant level of asymmetry $(>10 \%)$ in lower extremity gait parameters [125].Therefore, it makes sense that the healthy population tested in the present study showed a notable level of asymmetry in the baseline CON condition. It is also unsurprising that the addition of the DAO and DUAFO affected the asymmetry levels relative to CON. When used in healthy populations, foot orthotics have been shown to disturb gait symmetry. Guillebastre et al. showed that wearing an AFO can reduce walking velocity, step length, and step time in a healthy individual [92]. Gulgin et al. performed a 3D gait analysis in forty individuals with and without the presence of an orthopedic walking boot and found that the presence of the walking boot caused gait asymmetry similar to that of individuals with a leg length discrepancy [128]. However, AFO usage has been shown to have positive effects on the gait symmetry of individuals with structural deformities such as pes planus [129] and disorders involving afflicted motor control such as hemiparesis following stroke [130]. Gait asymmetry has not been assessed in ankle osteoarthritis specifically to our knowledge, but between-limb kinematic gait asymmetry has been recorded in cases of unilateral and bilateral mild to moderate knee osteoarthritis [131]. 
Table D-1. $\quad$ Preferred Speed During Over-Ground Walking and Spatio-Temporal Variables in Each Orthosis Condition During Set Speed Treadmill Walking

\begin{tabular}{|c|c|c|c|c|c|}
\hline Variable & Limb & $\mathrm{CON}$ & DAO & DUAFO & p-Value \\
\hline $\begin{array}{l}\text { Preferred } \\
\text { Speed }\left(\mathrm{m} \cdot \mathrm{s}^{-1}\right)\end{array}$ & N/A & $1.20 \pm 0.14$ & $1.12 \pm 0.14$ & $1.17 \pm 0.13$ & 0.288 \\
\hline \multirow{3}{*}{$\begin{array}{l}\text { Stance Time } \\
(\mathrm{s})^{*}\end{array}$} & Braced & $0.66 \pm 0.02$ & $0.71 \pm 0.03^{\mathrm{a}, \mathrm{c}}$ & $0.67 \pm 0.02$ & $<0.001$ \\
\hline & Unbraced & $0.66 \pm 0.03$ & $0.68 \pm 0.03^{a}$ & $0.67 \pm 0.02^{a}$ & 0.016 \\
\hline & Asymmetry & $1 \pm 1 \%$ & $4 \pm 3 \%$ & $1 \pm 0 \%$ & N/A \\
\hline \multirow{3}{*}{$\begin{array}{l}\text { Step Length } \\
\text { (m) }\end{array}$} & Braced & $0.75 \pm 0.03$ & $0.75 \pm 0.03$ & $0.76 \pm 0.03$ & 0.196 \\
\hline & Unbraced & $0.72 \pm 0.03$ & $0.74 \pm 0.04$ & $0.74 \pm 0.05$ & 0.297 \\
\hline & Asymmetry & $5 \pm 4 \%$ & $5 \pm 5 \%$ & $6 \pm 5 \%$ & N/A \\
\hline \multirow{3}{*}{$\begin{array}{l}\text { Cadence } \\
\left(\text { steps } \cdot \mathrm{min}^{-1}\right) *\end{array}$} & Braced & $114 \pm 5$ & $112 \pm 5$ & $111 \pm 3$ & 0.134 \\
\hline & Unbraced & $113 \pm 4$ & $110 \pm 3^{a, c}$ & $112 \pm 4$ & 0.021 \\
\hline & Asymmetry & $2 \pm 1 \%$ & $4 \pm 3 \%$ & $3 \pm 2 \%$ & $\mathrm{~N} / \mathrm{A}$ \\
\hline
\end{tabular}

Notes: Mean $\pm \mathrm{SD}$; *: orthosis effect; a: significant difference from CON; b: significant difference from DAO; $\mathrm{c}$ : significant difference from DUAFO. Significance: $\mathrm{p}<0.05$. Asymmetry Index: $0=$ no asymmetry, greater than $0=$ asymmetry 
Table D-2. Ankle Joint Kinematics in Each Orthosis Condition During Set Speed Treadmill Walking for Braced and Unbraced Limbs

\begin{tabular}{|c|c|c|c|c|c|}
\hline Variable & Limb & $\mathrm{CON}$ & DAO & DUAFO & p-Value \\
\hline \multirow{3}{*}{$\begin{array}{l}\text { Sagittal } \\
\text { Angle at } \\
\text { FS }\left(^{\circ}\right)\end{array}$} & Braced & $1.5 \pm 4.3$ & $-2.2 \pm 5.7$ & $-0.5 \pm 8.0$ & 0.217 \\
\hline & Unbraced & $6.1 \pm 5.3$ & $6.1 \pm 4.6$ & $5.2 \pm 6.0$ & 0.436 \\
\hline & Asymmetry & $109 \pm 77 \%$ & $192 \pm 123 \%$ & $318 \pm 586 \%$ & $\mathrm{~N} / \mathrm{A}$ \\
\hline \multirow{3}{*}{$\begin{array}{l}\text { ES PF } \\
\operatorname{ROM}\left(^{\circ}\right)\end{array}$} & Braced & $12.9 \pm 2.1$ & $9.7 \pm 4.3$ & $11.5 \pm 2.7$ & 0.066 \\
\hline & Unbraced & $14.9 \pm 4.0$ & $15.2 \pm 3.9$ & $15.4 \pm 4.6$ & 0.792 \\
\hline & Asymmetry & $22 \pm 16 \%$ & $34 \pm 28 \%$ & $27 \pm 20 \%$ & $\mathrm{~N} / \mathrm{A}$ \\
\hline \multirow{3}{*}{$\begin{array}{l}\text { ES Peak } \\
\text { PF }\left(^{\circ}\right)\end{array}$} & Braced & $-11.3 \pm 3.6$ & $-11.9 \pm 4.1$ & $-12.0 \pm 6.5$ & 0.951 \\
\hline & Unbraced & $-8.9 \pm 2.0$ & $-9.1 \pm 3.1$ & $-10.2 \pm 2.2$ & 0.264 \\
\hline & Asymmetry & $34 \pm 26 \%$ & $33 \pm 16 \%$ & $26 \pm 31 \%$ & $\mathrm{~N} / \mathrm{A}$ \\
\hline \multirow{3}{*}{$\begin{array}{l}\text { DF ROM } \\
\left(^{\circ}\right)\end{array}$} & Braced & $24.8 \pm 4.0$ & $23.0 \pm 3.3$ & $24.8 \pm 6.8$ & 0.414 \\
\hline & Unbraced & $22.1 \pm 3.9$ & $21.8 \pm 3.0$ & $22.6 \pm 3.2$ & 0.580 \\
\hline & Asymmetry & $16 \pm 12 \%$ & $10 \pm 10 \%$ & $14 \pm 19 \%$ & $\mathrm{~N} / \mathrm{A}$ \\
\hline \multirow{3}{*}{$\begin{array}{l}\text { ES Peak } \\
\text { PF AV } \\
\left({ }^{\circ} \cdot \mathrm{S}^{-1}\right)^{*}\end{array}$} & Braced & $-201.1 \pm 31.8$ & $-138.1 \pm 49.8^{a, c}$ & $-188.5 \pm 38.6$ & $<0.001$ \\
\hline & Unbraced & $-247.5 \pm 118.0$ & $-233.4 \pm 62.7$ & $-245.3 \pm 101.5$ & 0.934 \\
\hline & Asymmetry & $25 \pm 19 \%$ & $40 \pm 20 \%$ & $24 \pm 19 \%$ & $\mathrm{~N} / \mathrm{A}$ \\
\hline \multirow{3}{*}{$\begin{array}{l}\text { Peak EV } \\
\left(^{\circ}\right)\end{array}$} & Braced & $-9.4 \pm 2.8$ & $-9.3 \pm 3.4$ & $-7.0 \pm 1.9$ & 0.103 \\
\hline & Unbraced & $-7.3 \pm 3.2$ & $-8.8 \pm 2.5$ & $-8.3 \pm 2.8$ & 0.185 \\
\hline & Asymmetry & $42 \pm 41 \%$ & $38 \pm 33 \%$ & $24 \pm 17 \%$ & $\mathrm{~N} / \mathrm{A}$ \\
\hline \multirow{3}{*}{$\begin{array}{l}\text { EV ROM } \\
\left({ }^{\circ}\right)^{*}\end{array}$} & Braced & $11.0 \pm 2.8$ & $12.1 \pm 3.0$ & $4.6 \pm 2.0^{\mathrm{a}, \mathrm{b}}$ & $<0.001$ \\
\hline & Unbraced & $12.0 \pm 3.5$ & $12.7 \pm 4.3$ & $12.5 \pm 4.4$ & 0.626 \\
\hline & Asymmetry & $20 \pm 13 \%$ & $24 \pm 19 \%$ & $63 \pm 11 \%$ & $\mathrm{~N} / \mathrm{A}$ \\
\hline \multirow{3}{*}{$\begin{array}{l}\text { ES Peak } \\
\text { EV AV } \\
\left({ }^{\circ} \cdot \mathrm{s}^{-1}\right)^{*}\end{array}$} & Braced & $-171.5 \pm 49.9$ & $-112.2 \pm 48.3^{a}$ & $-59.5 \pm 27.1^{a, b}$ & $<0.001$ \\
\hline & Unbraced & $-178.6 \pm 58.4$ & $-186.9 \pm 84.7$ & $-189.4 \pm 80.2$ & 0.678 \\
\hline & Asymmetry & $19 \pm 11 \%$ & $42 \pm 21 \%$ & $65 \pm 21 \%$ & $\mathrm{~N} / \mathrm{A}$ \\
\hline
\end{tabular}

Notes: Mean \pm SD; *: orthosis effect; a: significant difference from CON; b: significant difference from DAO; $c$ : significant difference from DUAFO. Significance: $p<0.05$. ES: early stance; PF: plantarflexion; DF: dorsiflexion; EV: eversion; FS: foot strike; ROM: range of motion; AV: angular velocity. Asymmetry Index: $0=$ no asymmetry, greater than $0=$ asymmetry 
Table D-3. Knee and Hip Joint Kinematics in Each Orthosis Condition During Set Speed Treadmill Walking for Braced and Unbraced Limbs

\begin{tabular}{lllllc}
\hline Variable & Limb & CON & DAO & DUAFO & p-Value \\
\hline Knee Sagittal & Braced & $-2.3 \pm 4.3$ & $-5.8 \pm 6.7^{\text {a, c }}$ & $-1.2 \pm 4.6$ & 0.003 \\
Plane Contact & Unbraced & $-3.5 \pm 5.2$ & $-4.3 \pm 5.0$ & $-3.1 \pm 3.8$ & 0.253 \\
Angle $\left(^{\circ}\right)^{*}$ & Asymmetry & $22 \% \pm 107 \%$ & $38 \% \pm 114 \%$ & $97 \% \pm 250 \%$ & N/A \\
Knee Flexion & Braced & $15.2 \pm 6.4$ & $13.1 \pm 5.7$ & $19.0 \pm 4.1 \mathrm{~b}$ & 0.020 \\
ROM $\left(^{\circ}\right)^{*}$ & Unbraced & $15.7 \pm 5.3$ & $16.5 \pm 5.2$ & $16.3 \pm 4.8$ & 0.887 \\
& Asymmetry & $6 \% \pm 25 \%$ & $21 \% \pm 32 \%$ & $49 \% \pm 141 \%$ & N/A \\
Hip Sagittal & Braced & $32.7 \pm 7.1$ & $40.2 \pm 9.5$ a, c & $32.2 \pm 7.4$ & $<0.001$ \\
Plane Contact & Unbraced & $33.5 \pm 8.5$ & $37.4 \pm 8.5$ a, c & $32.7 \pm 7.5$ & 0.002 \\
Angle $\left(^{\circ}\right)^{*}$ & Asymmetry & $1 \% \pm 7 \%$ & $8 \% \pm 8 \%$ & $1 \% \pm 7 \%$ & N/A \\
Hip Extension & Braced & $40.4 \pm 4.6$ & $44.4 \pm 6.9$ a, c & $39.9 \pm 3.9$ & $<0.001$ \\
ROM $\left(^{\circ}\right)^{*}$ & Unbraced & $41.9 \pm 3.6$ & $44.2 \pm 4.8$ & $42.3 \pm 3.4$ & 0.071 \\
& Asymmetry & $4 \% \pm 5 \%$ & $0 \% \pm 9 \%$ & $6 \% \pm 6 \%$ & N/A \\
\hline
\end{tabular}

Notes: Mean $\pm \mathrm{SD}$; *: orthosis effect; a: significant difference from CON; b: significant difference from DAO; $c$ : significant difference from DUAFO. Significance: $p<0.05$. ROM: range of motion. Asymmetry Index: $0=$ no asymmetry, greater than $0=$ asymmetry 
The participants commonly reported the sensation of something heavy at the distal end of their leg while wearing the DAO, especially in the first few minutes after donning the DAO. This sensation is believed to be due in part to the distractive force placed across the ankle joint by the pneumatic cylinders, which may have disturbed the proprioception of the user and caused him to think that the brace was heavy. Ramakrishnan et al. compared gait asymmetry between multiple simulated physical changes (i.e., prosthetic limb, large and small leg length discrepancy, added mass at the distal end of the leg, and simulated stroke) and found that an increased mass $(2.3 \mathrm{~kg}$ or 4.6 $\mathrm{kg}$ ) at the distal end of the leg had a greater effect on gait asymmetry than a leg length discrepancy (27mm or 52mm) [93]. However, as noted previously (in Chapter 4), the DAO weighed slightly less than the DUAFO $(1.00 \mathrm{~kg}$ versus $1.04 \mathrm{~kg})$ and this sensation seemed to disappear with time, so this may have been only partially responsible for the kinematic asymmetries that were observed.

\section{CONCLUSIONS}

This work provided an initial glimpse into the effects of DAO and DUAFO brace wear on gait asymmetry of the joints of the lower limb. These findings showed that the healthy population tested in Chapter 4 presented natural asymmetrical kinematics at the ankle especially. Those asymmetries were affected by brace wear as we expected, although not always negatively (i.e., some variables showed improved asymmetry with brace wear). Kinematics of the knee and hip at initial contact were significantly affected by DAO wear, although the overall excursion of joint motion remained fairly similar or improved relative to CON. Further analysis of the effect of DAO wear on knee and hip joint kinematics and kinetics is warranted to provide a better understanding of its effect on walking symmetry and joint compensation. 


\section{APPENDIX E. EXTENDED METHODOLOGY OF THE CLINICAL STUDY}

This appendix provides additional information regarding the measurements taken and equipment used during the clinical study described in Chapter 5. Figures E-1 and E-2 show the FADI and VAS surveys used to quantify disability level and acute pain levels. Figures E-3 and E-4 show the force insoles and sample computed loading variables.

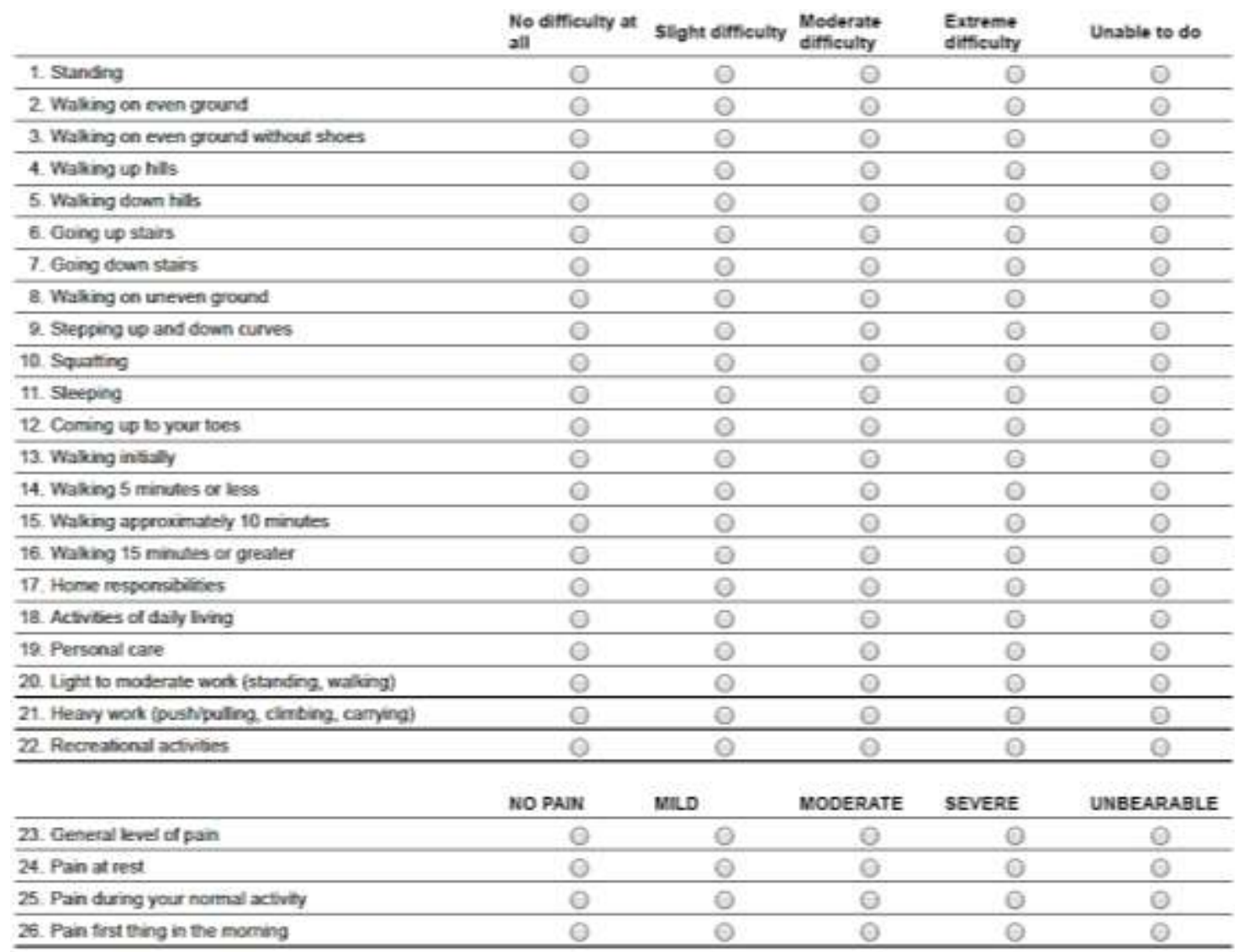

Figure E-1. Foot and Ankle Disability Index Score Survey Used to Quantify Disability Level at the Start of the Clinical Study

Foot and Ankle Disability Index (FADI). 

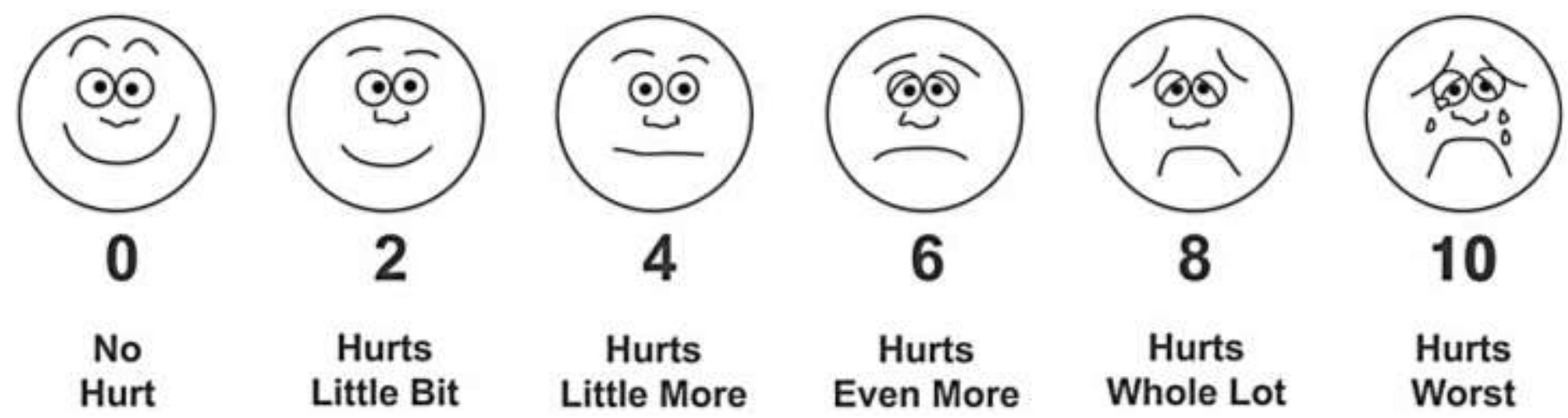

Hurts Whole Lot

Hurts Worst

Figure E-2. Visual Analog Scale Used to Quantify Pain Levels Following the Completion of Functional Activities in the Clinical Study

Visual Analog Scale (VAS). 


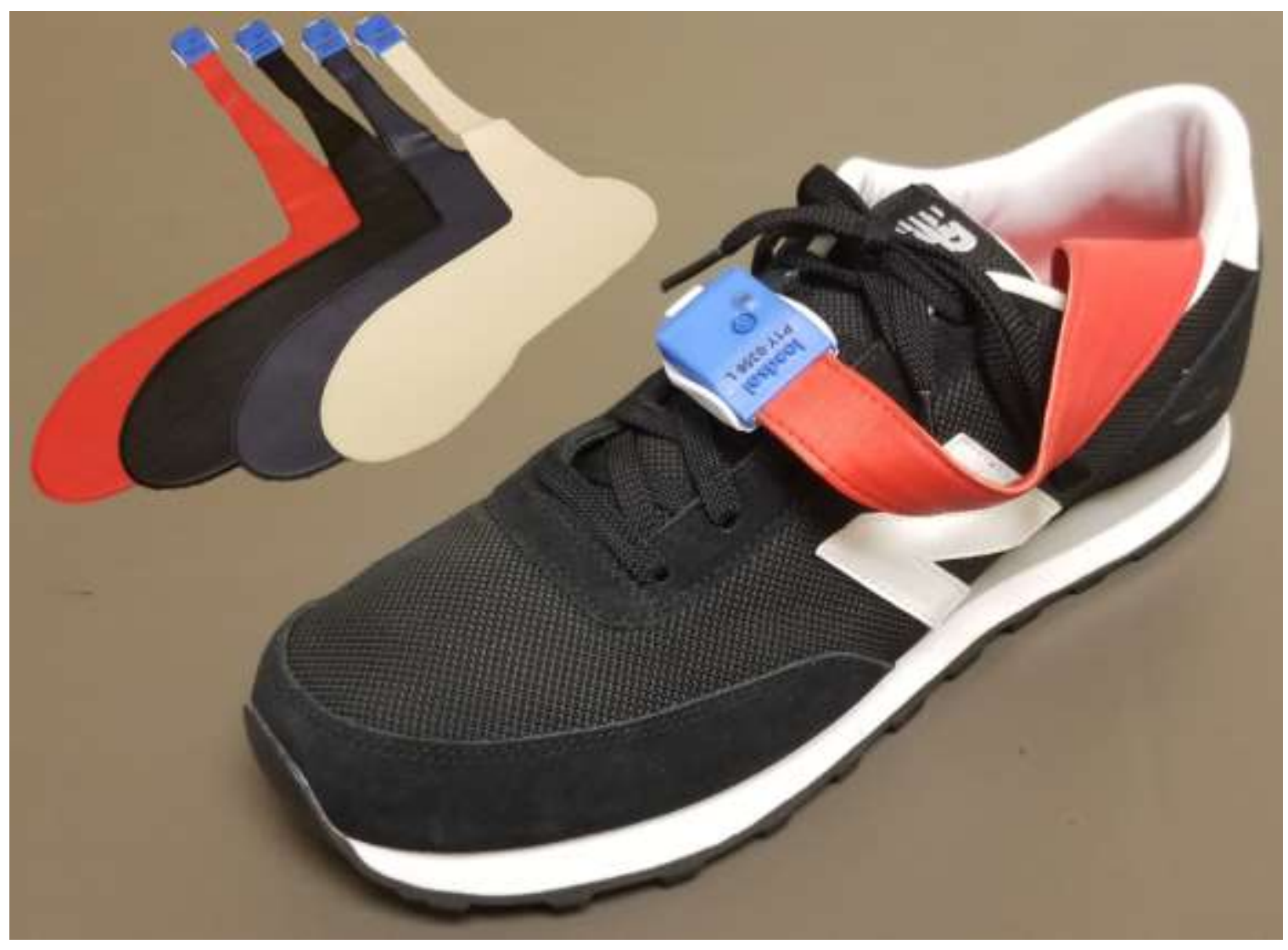

Figure E-3. Equipment Used During the Clinical Study

Standard footwear and insoles used to measure in-shoe vertical forces during gait. 

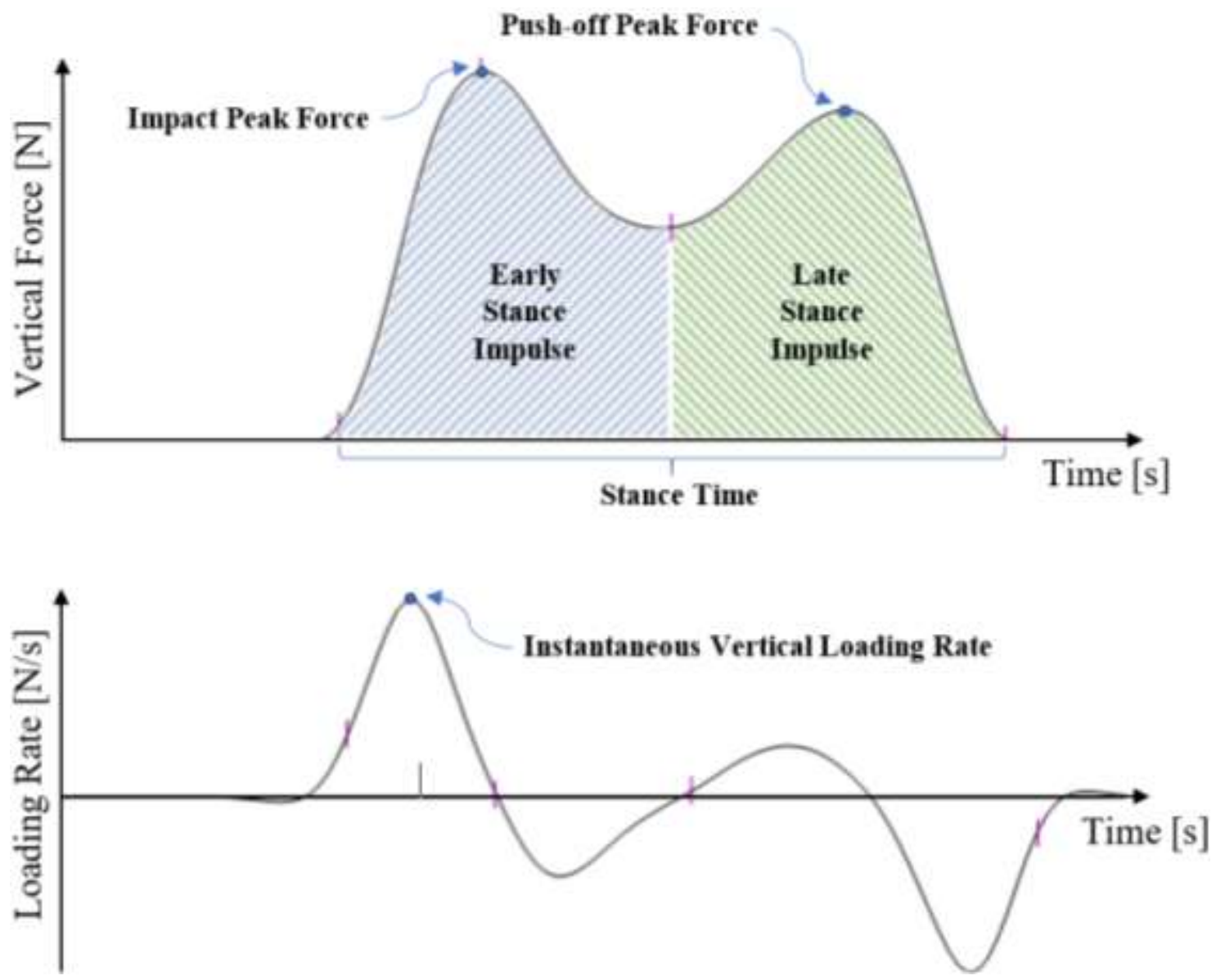

Figure E-4. Sample Vertical Force Parameters Derived from Insole Data 


\section{VITA}

Chloe was born in Schenectady, New York in 1990. She was raised in Memphis, Tennessee where she graduated from St. Benedict at Auburndale High School in 2008. She attended Christian Brothers University and graduated cum laude with a Bachelor of Science degree in Mechanical Engineering in 2013. She then attended graduate school at the University of Tennessee Health Science Center (UTHSC), where she was the recipient of the "Joe and Pat Johnson UTAA Scholarship" and "Edward and Bernice Humphrey Scholarship," among many other honors.

Chloe performed graduate research under the tutelage of her advisor Dr. Denis DiAngelo in the Orthopedic BioRobotics and Rehabilitation Laboratory, a wellestablished lab with a history of advanced biomechanical evaluation of surgical implants. Chloe's research topic extended beyond this scope to include the study and development of non-surgical medical devices. She graduated in 2015 with her Master of Science degree in Biomedical Engineering. For her early graduate research on studying and modeling the skeletal deformity, Scoliosis, and bracing technology, she received the "Outstanding Master Thesis" Award given by the Tennessee Conference of Graduate Schools.

Inspired by a passion for biomedical device design and biomechanical analysis, Chloe chose to pursue her doctorate and continue her research in the UTHSC Orthopedic BioRobotics and Rehabilitation Laboratory. During the course of her graduate work, Chloe collaborated with fellow scientists, clinicians, and practitioners to create new medical device technology for skeletal deformities and degenerative joint diseases of the spine, foot, and ankle. In 2017, she co-founded the start-up company "EMBrace Design" with her graduate advisor and a fellow graduate student to transition this new orthopedic technology out of the laboratory and into the market. In 2019, Chloe was honored with an international P.E.O. Scholar Award for her high level of academic achievement and her potential for having a positive impact on society. 